\title{
Synthesis of Bridged Oxabicycles via Cascade Reactions Involving $O$-Acyloxocarbenium Ion Intermediates
}

\author{
Hongjun Jeon, ${ }^{\text {a,b }}$ Sang Won Choi, ${ }^{a}$ Soojun Park, ${ }^{a}$ Seokwoo Lee, ${ }^{* a}$ and Sanghee Kim*a \\ ${ }^{a}$ College of Pharmacy, Seoul National University, 1 Gwanak-ro, Gwanak-gu, Seoul 08826, Republic of Korea \\ ${ }^{b}$ Therapeutics and Biotechnology Division, Korea Research Institute of Chemical Technology, 141 Gajeong- \\ ro, Yuseoung-gu, Daejeon 34114, Republic of Korea \\ pennkim@snu.ac.kr
}

Table of Contents
A. General Information
S2
B. Computational Study
S3 - S8
C. Experimental Procedures and Spectroscopic Data Analysis
S9-S21
D. X-ray Crystallographic Data
S22 - S24
E. NMR Study
S25 - S26
F. Copies of NMR data
S27 - S73 


\section{A. General Information}

All of the employed chemicals were of reagent grade and all of the reactions were performed under an inert atmosphere consisting of dry nitrogen using distilled dry solvents. The reactions were monitored by thin-layer chromatography (TLC) performed on silica gel $60 \mathrm{~F}_{254}$ TLC plates. The synthesized products were purified by flash column chromatography on silica gel 60 (40-63 $\mu \mathrm{m}, 230-400$ mesh, Merck). Melting points were measured on a Büchi B-545 apparatus (Büchi Labortechnik AG, Postfach, Switzerland). IR spectra were acquired using an Agilent 5500a FTIR (Agilent, USA). NMR spectra were obtained on JNM-ECZ400S/L1 spectrometers (Jeol, Akishima, Tokyo, Japan), a Bruker AVANCE 500 (Bruker, Rheinstetten, Germany), a Jeol JNM-ECA600 (Jeol, Tokyo, Japan), or an 800-MHz Bruker Avance III HD spectrometer with a 5-mm triple resonance inverse (TCI) CryoProbe (Bruker BioSpin, Germany). High resolution mass spectra were obtained with a JEOL JMS-700 double focusing magnetic sector mass spectrometer (JEOL, Tokyo, Japan) operated in the FAB, EI, or CI mode, or with Agilent Q-TOF 6530 (Santa Clara, CA, USA) operated in the ESI mode. Single crystal structure determination was performed using a SuperNova, Dual, $\mathrm{Cu}$ at home/near, AtlasS2 diffractometer (Agilent, USA). 


\section{B. Computational Study}

\section{Calculation of the electrophilicity using Fukui function}

\section{a) Theoretical background of Fukui function}

Local softness is one of the widely used local density functional descriptors to compare reactivity at different sites within one molecule. Local softness is given by

$$
s(r)=S f(r)
$$

where $\mathrm{S}$ is global softness and $f(r)$ is the Fukui function. ${ }^{1}$ The global softness can be approximated as

$$
S=1 /(\mathrm{IP}-\mathrm{EA})
$$

where IP and EA are the ionization potential and electron affinity respectively, of the chemical species.

The Fukui function describes the variation of the electronic density $(\rho(r))$ upon changing the number of electrons $(N)$ in the system. ${ }^{2}$

$$
f(r)=[\partial \rho(r) / \partial N]_{\Downarrow(r)}
$$

Thus, Fukui functions for nucleophilic and electrophilic attack on an atom, $\mathrm{k}$, in an $N$ electron system was introduced by Yang and Mortier $^{3}$ as

$$
\begin{aligned}
& f_{\mathrm{k}}{ }^{+}=\rho_{\mathrm{k}}(N+1)-\rho_{\mathrm{k}}(N) \text { (nucleophilic attack) } \\
& f_{\mathrm{k}}{ }^{-}=\rho_{\mathrm{k}}(N)-\rho_{\mathrm{k}}(N-1) \text { (electrophilic attack) }
\end{aligned}
$$

where $\rho_{\mathrm{k}}(N+1), \rho_{\mathrm{k}}(\mathrm{N})$ and $\rho_{\mathrm{k}}(N-1)$ are the electronic populations on atom $\mathrm{k}$ in the $N+1, N$ and $N-1$ electron systems. These functions can be condensed to the nuclei by using an atomic charge partitioning scheme, such as Mulliken ${ }^{4}$ population analysis. Therefore, the local softness for atom $\mathrm{k}$ can be written as

$$
\begin{aligned}
& s_{\mathrm{k}}{ }^{+}=\left[\rho_{\mathrm{k}}(N+1)-\rho_{\mathrm{k}}(N)\right] S \\
& s_{\mathrm{k}}{ }^{-}=\left[\rho_{\mathrm{k}}(N)-\rho_{\mathrm{k}}(N-1)\right] S
\end{aligned}
$$

Local softness is used as reactivity index, however, not always provides the correct reactivity trends. So new parameters are devised by S. Pal, ${ }^{5}$ which are "relative electrophilicity" and "relative nucleophilicity".

$$
\begin{aligned}
& \text { Relative electrophilicity }=s_{\mathrm{k}}{ }^{+} / s_{\mathrm{k}}{ }^{-} \\
& \text {Relative nucleophilicity }=s_{\mathrm{k}}{ }^{-} / s_{\mathrm{k}}{ }^{+}
\end{aligned}
$$

These two relative values indicate to the preferable reactive sites in the reaction.

b) Geometry optimization and energy minimization of $\boldsymbol{O}$-acyloxocabenium ion $\left(\mathrm{I}_{\mathrm{A}}\right)$, alkyloxocarbenium ion $\left(\mathrm{I}_{\mathrm{B}}\right)$,

\section{$N$-acyliminium ion $\left(\mathbf{I}_{\mathrm{C}}\right)$, and iminium ion $\left(\mathbf{I}_{\mathrm{D}}\right)$}

The computational energy minimizations of $\mathbf{I}_{\mathbf{A}}, \mathbf{I}_{\mathbf{B}}, \mathbf{I}_{\mathbf{C}}$, and $\mathbf{I}_{\mathbf{D}}$ were performed using the DMol3 program in Material Studio 2018. The Fukui function, ionization potential, and electron affinity were also calculated by using DMol3 program. ${ }^{6}$ In these calculations, we employed generalized gradient approximation (GGA) in the Perdew-Burke-

\footnotetext{
1 W. Yang, R. G. Parr. Hardness, softness, and the fukui function in the electronic theory of metals and catalysis. Proc. Natl. Acad. Sci. U.S.A. 1985, 82, 6723-6726.

${ }^{2}$ R. G. Parr, W. Yang. Density functional approach to the frontier-electron theory of chemical reactivity. J. Am. Chem. Soc. 1984, 106, 4049-4050.

3 W. Yang, W. J. Mortier. The use of global and local molecular parameters for the analysis of the gas-phase basicity of amines. J. Am. Chem. Soc. 1986, 108, 5708-5711.

${ }^{4}$ R. S. Mulliken. Electronic Population Analysis on LCAO-MO Molecular Wave Functions. I. J. Chem. Phys. 1955, 23, $1833-1840$.

5 R. K. Roy, S. Krishnamurti, P. Geerlings, S. Pal. Local Softness and Hardness Based Reactivity Descriptors for Predicting Intra- and Intermolecular Reactivity Sequences: Carbonyl Compounds. J. Phys. Chem. A 1998, 102, 3746-3755.

${ }^{6}$ (a) B. Delley. An all-electron numerical method for solving the local density functional for polyatomic molecules J. Chem. Phys. 1990, 92, 508-

517. (b) B. Delley. From molecules to solids with the DMol' ${ }^{3}$ approach. J. Chem. Phys. 2000, 113, 7756-7764.
} 
Ernzerhof $(\mathrm{PBE})^{7}$ form as well as double numerical polarized (DNP) basis sets. To calculate the Fukui function values, a Mulliken partitioning scheme was employed.

Table S1. Cartesian coordinates of $\mathbf{I}_{\mathbf{A}}, \mathbf{I}_{\mathbf{B}}, \mathbf{I}_{\mathbf{C}}$, and $\mathbf{I}_{\mathbf{D}}$

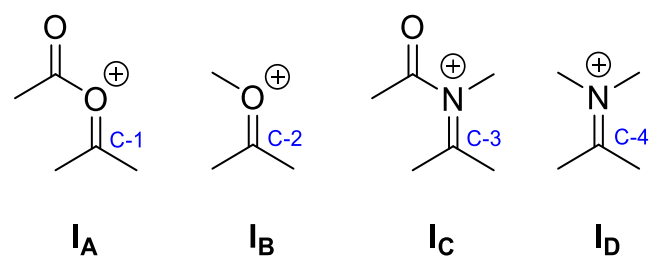

$\mathrm{I}_{\mathrm{A}}(\mathrm{O}$-acyloxocabenium ion $)$

\begin{tabular}{cccccccc}
\hline Atom & $\mathrm{X}$ & $\mathrm{Y}$ & $\mathrm{Z}$ & Atom & $\mathrm{X}$ & $\mathrm{Y}$ & $\mathrm{Z}$ \\
\hline $\mathrm{C}$ & -5.5989 & 0.6897 & -0.0144 & $\mathrm{H}$ & -2.6646 & 1.1996 & -1.2765 \\
$\mathrm{C}$ & -4.4163 & 1.6739 & -0.0322 & $\mathrm{H}$ & -2.9581 & 0.0425 & 0.1498 \\
$\mathrm{C}$ & -2.9835 & 1.1304 & -0.1842 & $\mathrm{H}$ & -2.2696 & 1.7437 & 0.4615 \\
$\mathrm{O}$ & -4.6353 & 3.1389 & 0.2712 & $\mathrm{O}$ & -6.2014 & 5.1659 & 0.786 \\
$\mathrm{C}$ & -6.0277 & 3.7096 & 0.4287 & $\mathrm{C}$ & -7.2722 & 2.8248 & 0.2264 \\
$\mathrm{H}$ & -5.1977 & -0.3738 & 0.0132 & $\mathrm{H}$ & -7.195 & 1.8824 & 0.868 \\
$\mathrm{H}$ & -6.2316 & 0.8366 & -0.9512 & $\mathrm{H}$ & -7.3539 & 2.5344 & -0.8719 \\
$\mathrm{H}$ & -6.2378 & 0.8769 & 0.9144 & $\mathrm{H}$ & -8.1982 & 3.4113 & 0.5326 \\
\hline
\end{tabular}

\section{IB (alkyloxocarbenium ion)}

\begin{tabular}{cccccccc}
\hline Atom & $\mathrm{X}$ & $\mathrm{Y}$ & $\mathrm{Z}$ & Atom & $\mathrm{X}$ & $\mathrm{Y}$ & $\mathrm{Z}$ \\
\hline $\mathrm{C}$ & -0.7949 & -1.1224 & -0.0712 & $\mathrm{H}$ & -1.6255 & -0.7 & 0.54 \\
$\mathrm{C}$ & 0.4281 & -0.237 & -0.0459 & $\mathrm{H}$ & 2.2505 & -0.6578 & -1.0961 \\
$\mathrm{C}$ & 1.8493 & -0.7263 & -0.0538 & $\mathrm{H}$ & 1.869 & -1.7825 & 0.2844 \\
$\mathrm{O}$ & 0.3081 & 1.1395 & 0.1312 & $\mathrm{H}$ & 2.4946 & -0.0943 & 0.603 \\
$\mathrm{C}$ & -1.0438 & 1.7599 & 0.1304 & $\mathrm{H}$ & -1.8255 & 1.0428 & 0.4603 \\
$\mathrm{H}$ & -0.5366 & -2.1326 & 0.3097 & $\mathrm{H}$ & -1.2493 & 2.1151 & -0.9045 \\
$\mathrm{H}$ & -1.1589 & -1.2175 & -1.1256 & $\mathrm{H}$ & -0.9651 & 2.6131 & 0.838 \\
\hline
\end{tabular}

IC $(\mathrm{N}$-acyliminium ion)

\begin{tabular}{cccccccc}
\hline Atom & $\mathrm{X}$ & $\mathrm{Y}$ & $\mathrm{Z}$ & Atom & $\mathrm{X}$ & $\mathrm{Y}$ & $\mathrm{Z}$ \\
\hline $\mathrm{C}$ & -0.537 & -1.8836 & -0.219 & $\mathrm{H}$ & 2.6229 & -0.4389 & 0.2451 \\
$\mathrm{C}$ & 0.5 & -0.7606 & -0.1949 & $\mathrm{O}$ & -1.0747 & 2.7637 & 0.5817 \\
$\mathrm{C}$ & 1.9664 & -1.1733 & -0.3112 & $\mathrm{C}$ & 1.3977 & 1.5926 & 0.2364 \\
$\mathrm{~N}$ & 0.2139 & 0.6799 & 0.0687 & $\mathrm{H}$ & 1.6734 & 2.0464 & -0.7635 \\
$\mathrm{C}$ & -1.1223 & 1.3435 & 0.2675 & $\mathrm{H}$ & 2.2746 & 1.0035 & 0.6399 \\
$\mathrm{H}$ & 0.0043 & -2.8777 & -0.211 & $\mathrm{H}$ & 1.1243 & 2.4164 & 0.9627 \\
$\mathrm{H}$ & -1.1672 & -1.8079 & -1.1556 & $\mathrm{C}$ & -2.5004 & 0.6913 & 0.134 \\
$\mathrm{H}$ & -1.2022 & -1.8152 & 0.6913 & $\mathrm{H}$ & -2.6394 & -0.1015 & 0.9272 \\
$\mathrm{H}$ & 2.2635 & -1.1871 & -1.4049 & $\mathrm{H}$ & -2.6087 & 0.2243 & -0.89 \\
$\mathrm{H}$ & 2.0975 & -2.2085 & 0.1276 & $\mathrm{H}$ & -3.2868 & 1.4929 & 0.2681 \\
\hline
\end{tabular}

ID (iminium ion)

\begin{tabular}{cccccccc}
\hline Atom & $\mathrm{X}$ & $\mathrm{Y}$ & $\mathrm{Z}$ & Atom & $\mathrm{X}$ & $\mathrm{Y}$ & $\mathrm{Z}$ \\
\hline $\mathrm{C}$ & -1.1078 & -1.6303 & -0.1003 & $\mathrm{H}$ & 1.5293 & -2.2682 & 0.2547 \\
$\mathrm{C}$ & 0.092 & -0.7035 & -0.0757 & $\mathrm{H}$ & 2.1748 & -0.6039 & 0.582 \\
$\mathrm{C}$ & 1.5157 & -1.2119 & -0.0814 & $\mathrm{H}$ & -2.2266 & 0.5304 & 0.429 \\
$\mathrm{~N}$ & -0.1083 & 0.6595 & 0.1061 & $\mathrm{H}$ & -1.7101 & 1.6332 & -0.9168 \\
$\mathrm{C}$ & -1.465 & 1.2692 & 0.1074 & $\mathrm{H}$ & -1.4496 & 2.1244 & 0.8176 \\
$\mathrm{H}$ & -0.8183 & -2.6298 & 0.2841 & $\mathrm{C}$ & 1.0483 & 1.5668 & 0.0894 \\
\hline
\end{tabular}

7 J. P. Perdew, K. Burke, M. Ernzerhof. Generalized Gradient Approximation Made Simple. Phys. Rev. Lett. 1996, 77, 3865-3868. 


\begin{tabular}{cccccccc}
\hline $\mathrm{H}$ & -1.4698 & -1.745 & -1.1511 & $\mathrm{H}$ & 1.2563 & 1.9044 & -0.9528 \\
$\mathrm{H}$ & -1.9538 & -1.2438 & 0.5105 & $\mathrm{H}$ & 1.9457 & 1.05 & 0.4934 \\
$\mathrm{H}$ & 1.9298 & -1.1538 & -1.1172 & $\mathrm{H}$ & 0.8174 & 2.4522 & 0.7211 \\
\hline
\end{tabular}

Table S2. Molecular energy of $\mathbf{I}_{\mathbf{A}}, \mathbf{I}_{\mathbf{B}}, \mathbf{I}_{\mathbf{C}}$, and $\mathbf{I}_{\mathbf{D}}$

\begin{tabular}{ccccc}
\hline Molecule & Hartree $(\mathrm{Ha})^{a}$ & $\begin{array}{c}\text { Ionizaion potential } \\
(\mathrm{eV})\end{array}$ & $\begin{array}{c}\text { Electron affinity } \\
(\mathrm{eV})\end{array}$ & $\begin{array}{c}\text { Global softness } \\
\left(\mathrm{eV}^{-1}\right)^{b}\end{array}$ \\
\hline $\mathbf{I}_{\mathbf{A}}$ & -345.86185 & 14.69700512 & 5.208531212 & 0.105391026 \\
$\mathbf{I}_{\mathbf{B}}$ & -232.610434 & 15.96720537 & 4.373685919 & 0.086255085 \\
$\mathbf{I}_{\mathbf{C}}$ & -365.286943 & 13.81312491 & 4.513443593 & 0.107530566 \\
$\mathbf{I}_{\mathbf{D}}$ & -252.042566 & 14.21713235 & 3.423872524 & 0.092650415 \\
\hline${ }^{a} 1 \mathrm{Ha}=627.509391 \mathrm{kcal} / \mathrm{mol} .{ }^{b}$ Global softness $=1 /$ (Ionization potential - Electron affinity).
\end{tabular}

Table S3. Relative electrophilicity of C-1, C-2, C-3, and C-4

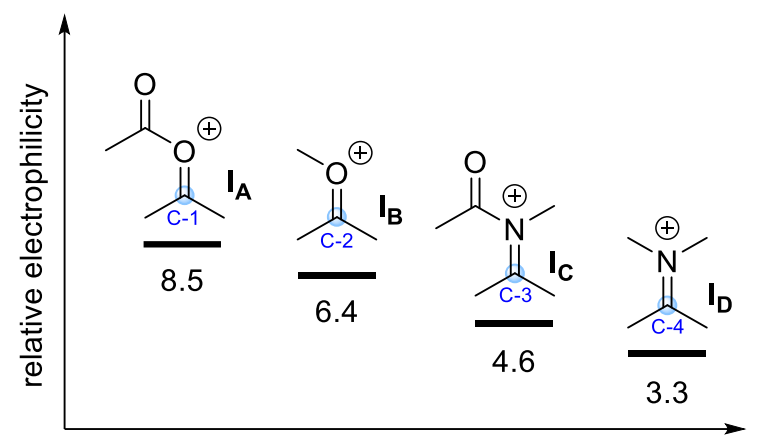

\begin{tabular}{cccccc}
\hline Atom & $\begin{array}{c}\text { Fukui function } \\
(-)\end{array}$ & $\begin{array}{c}\text { Fukui function } \\
(+)\end{array}$ & $\begin{array}{c}\text { Local softness } \\
(\mathrm{S}-)^{a}\end{array}$ & $\begin{array}{c}\text { Local softness } \\
(\mathrm{S}+)\end{array}$ & $\begin{array}{c}\text { Relative } \\
\text { electrophilicity }^{b}\end{array}$ \\
\hline $\mathrm{C}-1$ & $\mathbf{0 . 0 2 4}$ & $\mathbf{0 . 2 0 3}$ & $\mathbf{0 . 0 0 2 5}$ & $\mathbf{0 . 0 2 1 4}$ & $\mathbf{8 . 5}$ \\
$\mathrm{C}-2$ & $\mathbf{0 . 0 3 7}$ & $\mathbf{0 . 2 3 7}$ & $\mathbf{0 . 0 0 3 2}$ & $\mathbf{0 . 0 2 0 4}$ & $\mathbf{6 . 4}$ \\
$\mathrm{C}-3$ & $\mathbf{0 . 0 3 7}$ & $\mathbf{0 . 1 7 1}$ & $\mathbf{0 . 0 0 4 0}$ & $\mathbf{0 . 0 1 8 4}$ & $\mathbf{4 . 6}$ \\
$\mathrm{C}-4$ & $\mathbf{0 . 0 6 3}$ & $\mathbf{0 . 2 0 7}$ & $\mathbf{0 . 0 0 5 8}$ & $\mathbf{0 . 0 1 9 2}$ & $\mathbf{3 . 3}$ \\
\hline
\end{tabular}

${ }^{a}$ Local softness $=$ Global softness $\times$ Fukui function. ${ }^{b}$ Relative electrophilicity $=$ Local softness $(\mathrm{S}+) /$ Local softness $(\mathrm{S}-)$.

\section{Calculation for AOI involved Friedel-Crafts reaction (Figure 2b)}

\section{a) General procedure for molecular energy calculations}

Density functional theory (DFT) calculations were carried out using PBEPBE functionals with the 6-311 G(d,p) basis set in Gaussian 16. Transition-state optimizations were performed with the Berny geometry optimization algorithm. Frequency calculations were carried out to ensure that minima structures had no negative frequency and that the transition structures had only one imaginary frequency and to calculate the contributions towards the Gibbs free energy (reported at $298.15 \mathrm{~K}$ and $1 \mathrm{~atm}$ ). The connectivity of reactants and products was confirmed by intrinsic reaction coordination (IRC) calculations. All molecules were modeled in the solvent phase $\left(\mathrm{CH}_{2} \mathrm{Cl}_{2}\right.$, solvation model based on density (SMD)). 


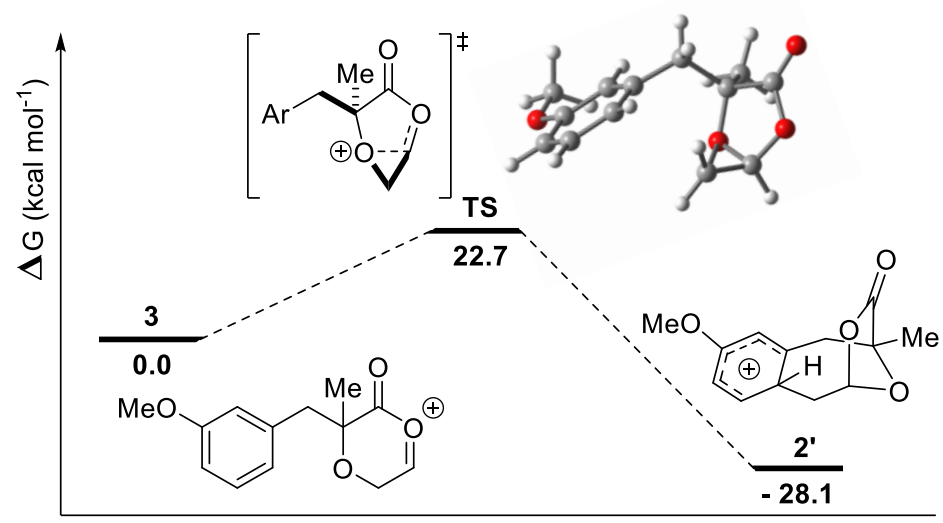

Table S4. Electronic energies (E), zero-point energies (ZPE), enthalpies (H), and Gibbs free energies (G) (in Hartree, $\mathrm{Ha}$ ) of the compound calculated at the PBEPBE, 6-311 G(d,p) level of theory. The relative Gibbs free energy is shown in $\mathrm{kcal} / \mathrm{mol}$, and the imaginary frequency is shown in $\mathrm{cm}^{-1}$.

\begin{tabular}{ccccccc}
\hline Molecule & $\mathrm{E}$ & $\mathrm{ZPE}$ & $\mathrm{H}$ & $\mathrm{G}$ & $\Delta G^{a, b}$ & $\begin{array}{c}\text { imaginary } \\
\text { frequency }\end{array}$ \\
\hline $\mathbf{3}$ & -804.306197 & -804.056114 & -804.038364 & -804.100922 & 0.0 & - \\
TS & -804.271735 & -804.021551 & -804.004854 & -804.064708 & 22.7 & -251.40 \\
$\mathbf{2}^{\prime}$ & -804.359250 & -804.104137 & -804.088159 & -804.145687 & -28.1 & - \\
\hline \multicolumn{7}{c}{${ }^{a} 1 \mathrm{Ha}=627.509391 \mathrm{kcal} / \mathrm{mol}}$. \\
\end{tabular}

Table S5. Cartesian coordinates of the $\mathbf{3}$, TS and $\mathbf{2}^{\prime}$.

\begin{tabular}{cccccccc}
3 & \multicolumn{7}{l}{} \\
\hline Atom & $\mathrm{X}$ & $\mathrm{Y}$ & $\mathrm{Z}$ & Atom & $\mathrm{X}$ & $\mathrm{Y}$ & $\mathrm{Z}$ \\
\hline $\mathrm{C}$ & 2.162631 & 2.299531 & -0.21742 & $\mathrm{H}$ & 1.83424 & -1.47283 & 0.664717 \\
$\mathrm{C}$ & 3.298138 & 1.493439 & -0.22921 & $\mathrm{H}$ & -0.38804 & -1.19932 & 1.237035 \\
$\mathrm{C}$ & 3.190142 & 0.125051 & 0.087496 & $\mathrm{H}$ & -1.0557 & 0.398194 & 1.548502 \\
$\mathrm{C}$ & 1.934635 & -0.41679 & 0.410717 & $\mathrm{C}$ & -0.79474 & -1.06062 & -1.64421 \\
$\mathrm{C}$ & 0.7914 & 0.404038 & 0.422549 & $\mathrm{H}$ & -3.41128 & 2.318526 & -0.70182 \\
$\mathrm{C}$ & 0.906842 & 1.765387 & 0.105418 & $\mathrm{H}$ & -2.19968 & 2.281849 & 0.570748 \\
$\mathrm{C}$ & -0.54548 & -0.20835 & 0.783191 & $\mathrm{O}$ & -2.96072 & -2.4048 & -0.08847 \\
$\mathrm{C}$ & -1.48619 & -0.40158 & -0.45013 & $\mathrm{H}$ & -0.35929 & -2.02604 & -1.35595 \\
$\mathrm{O}$ & -2.05072 & 0.826003 & -0.97 & $\mathrm{H}$ & 0.008161 & -0.39071 & -1.97722 \\
$\mathrm{C}$ & -2.80849 & 1.621402 & -0.08949 & $\mathrm{H}$ & -1.50932 & -1.20844 & -2.46399 \\
$\mathrm{C}$ & -3.73165 & 0.888738 & 0.787661 & $\mathrm{H}$ & -4.41548 & 1.44179 & 1.452394 \\
$\mathrm{O}$ & -3.77583 & -0.36245 & 0.844968 & $\mathrm{H}$ & 0.032009 & 2.417529 & 0.113899 \\
$\mathrm{C}$ & -2.63251 & -1.29076 & -0.03784 & $\mathrm{C}$ & 4.295865 & -1.98407 & 0.366806 \\
$\mathrm{H}$ & 2.254779 & 3.360632 & -0.45867 & $\mathrm{H}$ & 5.325932 & -2.34733 & 0.278199 \\
$\mathrm{H}$ & 4.281949 & 1.897639 & -0.47499 & $\mathrm{H}$ & 3.652701 & -2.52645 & -0.34612 \\
$\mathrm{O}$ & 4.353611 & -0.58439 & 0.056628 & $\mathrm{H}$ & 3.934663 & -2.15253 & 1.394956 \\
\hline
\end{tabular}

\begin{tabular}{|c|c|c|c|c|c|c|c|}
\hline Atom & $X$ & $\mathrm{Y}$ & $Z$ & Atom & $\mathrm{X}$ & $\mathrm{Y}$ & $\mathrm{Z}$ \\
\hline $\mathrm{C}$ & 1.942672 & -2.25851 & -0.51685 & $\mathrm{H}$ & 1.655137 & 1.634089 & -0.40814 \\
\hline $\mathrm{C}$ & 2.99963 & -1.5105 & -0.02604 & $\mathrm{H}$ & -0.4818 & 1.361997 & -1.86488 \\
\hline $\mathrm{C}$ & 2.90264 & -0.09613 & 0.008555 & $\mathrm{H}$ & -1.20747 & -0.24507 & -1.96225 \\
\hline $\mathrm{C}$ & 1.726019 & 0.546684 & -0.42673 & $\mathrm{C}$ & -1.35082 & 2.250775 & 0.404018 \\
\hline $\mathrm{C}$ & 0.651249 & -0.21388 & -0.9006 & $\mathrm{H}$ & -0.84397 & -2.48505 & 1.500837 \\
\hline
\end{tabular}




\begin{tabular}{lccccccc}
\hline $\mathrm{C}$ & 0.766581 & -1.62149 & -0.96114 & $\mathrm{H}$ & -1.70891 & -2.37382 & -0.17619 \\
$\mathrm{C}$ & -0.65031 & 0.434036 & -1.29979 & $\mathrm{O}$ & -3.73944 & 1.010952 & -1.1593 \\
$\mathrm{C}$ & -1.54138 & 0.807864 & -0.0631 & $\mathrm{H}$ & -1.65763 & 2.921375 & -0.41191 \\
$\mathrm{O}$ & -1.23993 & -0.03288 & 1.094097 & $\mathrm{H}$ & -0.29515 & 2.436368 & 0.642792 \\
$\mathrm{C}$ & -1.57113 & -2.01148 & 0.84113 & $\mathrm{H}$ & -1.96269 & 2.462363 & 1.290864 \\
$\mathrm{C}$ & -2.39706 & -0.90788 & 1.347377 & $\mathrm{H}$ & -2.685 & -0.89153 & 2.403719 \\
$\mathrm{O}$ & -3.44814 & -0.49273 & 0.521397 & $\mathrm{H}$ & -0.00323 & -2.20545 & -1.46764 \\
$\mathrm{C}$ & -3.01905 & 0.509217 & -0.34536 & $\mathrm{C}$ & 3.987374 & 1.982503 & 0.535491 \\
$\mathrm{H}$ & 2.02786 & -3.34486 & -0.57853 & $\mathrm{H}$ & 4.966143 & 2.259211 & 0.94053 \\
$\mathrm{H}$ & 3.925212 & -1.97603 & 0.316003 & $\mathrm{H}$ & 3.19136 & 2.342861 & 1.205413 \\
$\mathrm{O}$ & 3.99474 & 0.542292 & 0.475904 & $\mathrm{H}$ & 3.863896 & 2.415219 & -0.4693 \\
\hline
\end{tabular}

\begin{tabular}{cccccccc}
$\mathbf{2}^{\prime}$ & \multicolumn{7}{c}{ ( } \\
\hline Atom & $\mathrm{X}$ & $\mathrm{Y}$ & $\mathrm{Z}$ & Atom & $\mathrm{X}$ & $\mathrm{Y}$ & $\mathrm{Z}$ \\
\hline $\mathrm{C}$ & -1.452 & 2.002923 & -0.48372 & $\mathrm{H}$ & -1.98919 & -1.847 & 0.000539 \\
$\mathrm{C}$ & -2.71521 & 1.524541 & -0.38106 & $\mathrm{H}$ & 0.254489 & -2.28775 & 0.06621 \\
$\mathrm{C}$ & -2.90869 & 0.123405 & -0.14497 & $\mathrm{H}$ & 0.695887 & -1.5501 & -1.47185 \\
$\mathrm{C}$ & -1.80404 & -0.77748 & -0.10027 & $\mathrm{C}$ & 2.827129 & -2.23368 & 0.373584 \\
$\mathrm{C}$ & -0.51671 & -0.3235 & -0.24042 & $\mathrm{H}$ & -0.06279 & 1.558117 & 1.827183 \\
$\mathrm{C}$ & -0.24382 & 1.147898 & -0.32988 & $\mathrm{H}$ & 0.65762 & 2.800221 & 0.774395 \\
$\mathrm{C}$ & 0.58421 & -1.34191 & -0.38967 & $\mathrm{O}$ & 3.200702 & -0.13924 & -1.79977 \\
$\mathrm{C}$ & 1.976377 & -0.98381 & 0.18085 & $\mathrm{H}$ & 2.96157 & -2.74637 & -0.58928 \\
$\mathrm{O}$ & 1.8599 & -0.28664 & 1.437039 & $\mathrm{H}$ & 2.33107 & -2.91731 & 1.077093 \\
$\mathrm{C}$ & 0.535584 & 1.716204 & 0.91815 & $\mathrm{H}$ & 3.813982 & -1.96365 & 0.773057 \\
$\mathrm{C}$ & 1.911607 & 1.098542 & 1.137945 & $\mathrm{H}$ & 2.439473 & 1.612208 & 1.953378 \\
$\mathrm{O}$ & 2.711208 & 1.238171 & -0.06315 & $\mathrm{H}$ & 0.414185 & 1.333282 & -1.20384 \\
$\mathrm{C}$ & 2.704252 & 0.035492 & -0.71417 & $\mathrm{C}$ & -4.51186 & -1.6382 & 0.245824 \\
$\mathrm{H}$ & -1.28148 & 3.071878 & -0.63185 & $\mathrm{H}$ & -5.59935 & -1.63006 & 0.356742 \\
$\mathrm{H}$ & -3.59586 & 2.16287 & -0.45688 & $\mathrm{H}$ & -4.03326 & -1.9839 & 1.171227 \\
$\mathrm{O}$ & -4.16031 & -0.24452 & -0.00745 & $\mathrm{H}$ & -4.2194 & -2.25718 & -0.61273 \\
\hline
\end{tabular}




\section{Conformational search of $\boldsymbol{O}$-acyloxocarbenium ion 3}

A conformational search was performed using a mixed sampling method of torsional/low-mode using Macromodel (version 9.9, Schrödinger LLC) with the Merk molecular force field (MMFF). The upper limit of the relative potential energy was $10 \mathrm{~kJ} / \mathrm{mol}$, and a convergence threshold of $0.001 \mathrm{~kJ}(\mathrm{~mol} \AA)^{-1}$ for the rms gradient was used to minimize computational complexity and expense. A total of 24 conformers were obtained. The Boltzmann population of each conformer was calculated.

Table S6. Conformation of $O$-acyloxocarbenium ion $\mathbf{3}$

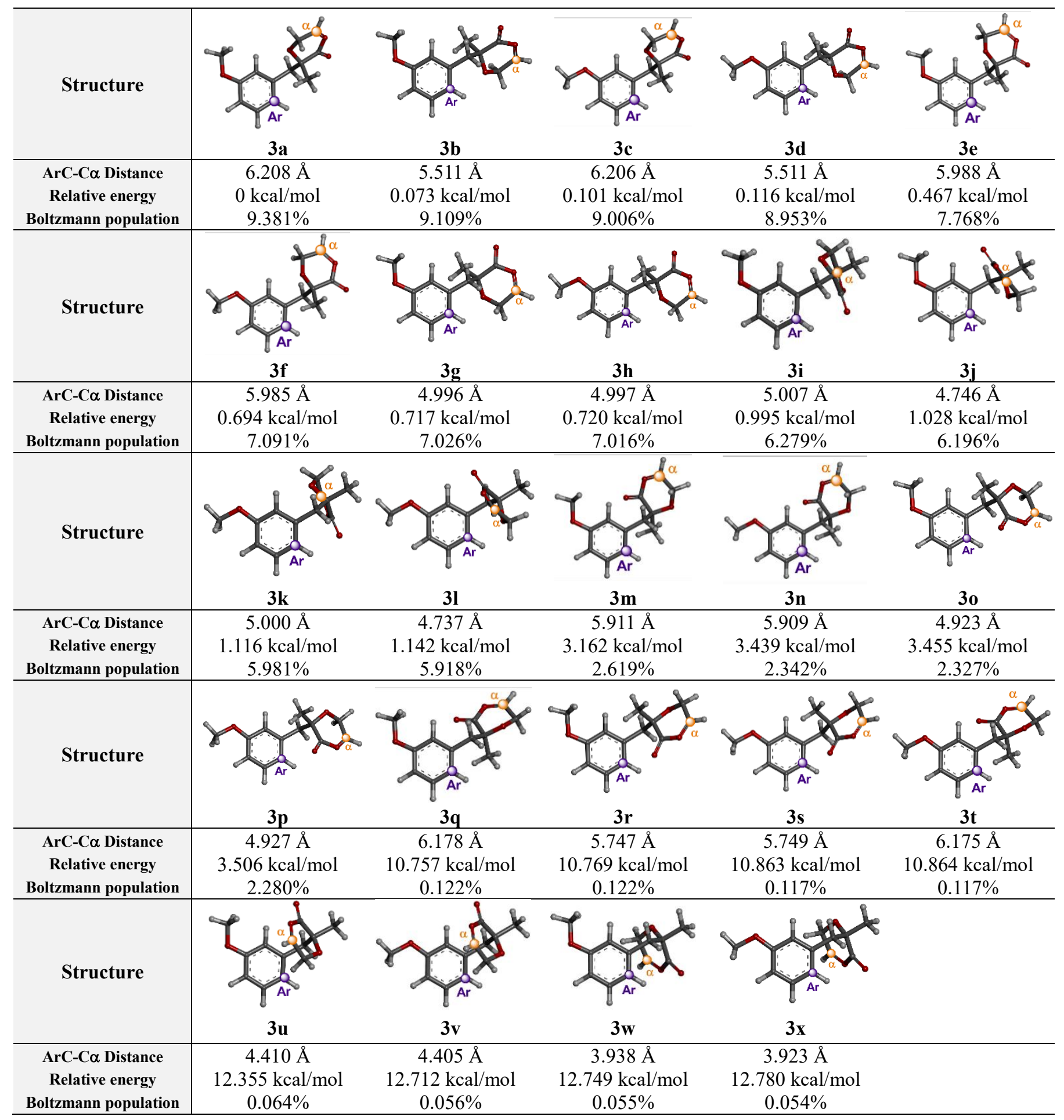




\section{B. Experimental Procedures and Spectroscopic Data Analysis}

\section{Friedel-Crafts reaction substrates}
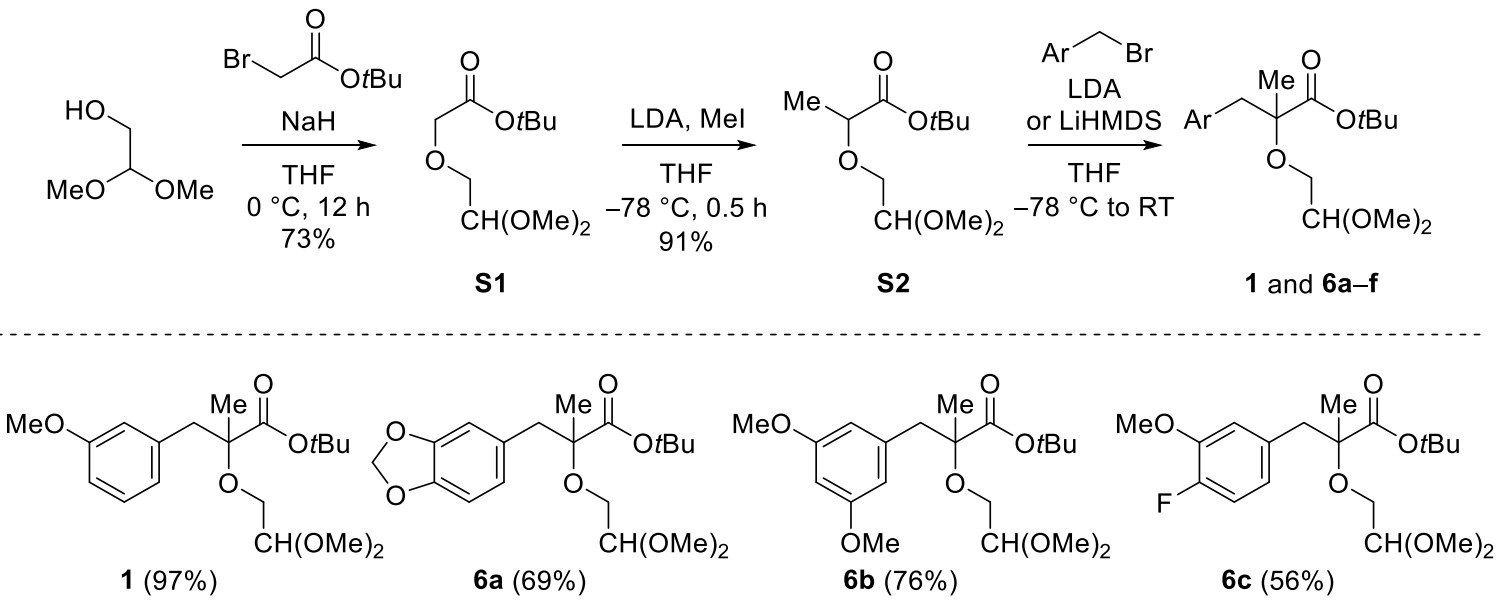<smiles>CCOC(=O)C(C)(Cc1cccc(C)c1)OCCC(C)C</smiles>

$6 d(76 \%)$

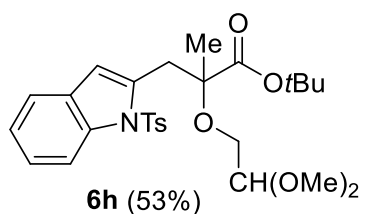

6h $(53 \%)$<smiles>CCCOC(=O)C(C)(Cc1ccccc1)OCC(C)C</smiles>

$6 e(68 \%)$

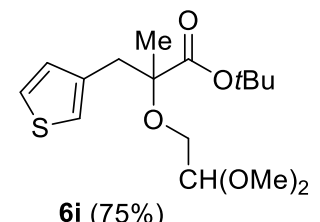<smiles>CCOC(=O)C(C)(Cc1ccc2ccccc2c1)OCCC(C)C</smiles>

6f $(68 \%)$

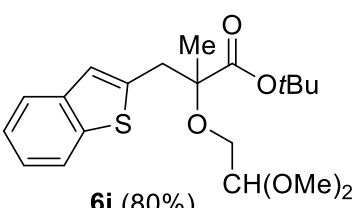<smiles>CCCCOC(C)(Cc1cccc(Br)c1)C(=O)OCC(C)C</smiles>

$6 \mathrm{~g}(23 \%)$

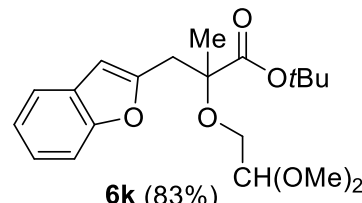

Scheme S1. Synthesis of Friedel-Crafts substrates $\mathbf{1}$ and $\mathbf{6 a}-\mathbf{k}$

tert-Butyl 2-(2,2-dimethoxyethoxy)acetate (S1). tert-Butyl 2-bromoacetate (1.2 equiv, $83.1 \mathrm{mmol}, 12.2 \mathrm{~mL})$ was added to a solution of glycolaldehyde dimethyl acetal (1.0 equiv, $69.3 \mathrm{mmol}, 7 \mathrm{~mL})$ in tetrahydrofuran $(35 \mathrm{~mL})$ at $0{ }^{\circ} \mathrm{C}$, followed by the portionwise addition of sodium hydride (60\% dispersion in mineral oil, 3 equiv, $207.8 \mathrm{mmol}, 8.3 \mathrm{~g}$ ). The reaction was stirred at room temperature for $12 \mathrm{~h}$, quenched by saturated aqueous $\mathrm{NH}_{4} \mathrm{Cl}$, poured into water, extracted twice with EtOAc, dried over $\mathrm{MgSO}_{4}$ and then concentrated in vacuo. The crude mixture was purified by flash chromatography on silica gel (hexane/EtOAc, $\left.6: 1, \mathbf{R}_{\mathrm{f}}=0.3\right)$ to give $\mathbf{S 1}(50.6 \mathrm{mmol}, 11.1 \mathrm{~g}, 73 \%)$ as a colorless oil. ${ }^{1} \mathrm{H}$ $\operatorname{NMR}\left(\mathrm{CDCl}_{3}, 400 \mathrm{MHz}\right) \delta 4.48(\mathrm{t}, J=5.3 \mathrm{~Hz}, 1 \mathrm{H}), 3.94(\mathrm{~s}, 2 \mathrm{H}), 3.52(\mathrm{~d}, J=5.5 \mathrm{~Hz}, 2 \mathrm{H}), 3.33(\mathrm{~s}, 6 \mathrm{H}), 1.40(\mathrm{~s}, 9 \mathrm{H})$; ${ }^{13} \mathrm{C} \mathrm{NMR}\left(\mathrm{CDCl}_{3}, 100 \mathrm{MHz}\right) \delta 169.5,102.8,81.7,70.9,69.1,54.1$ (2C), $28.2(3 \mathrm{C})$; IR (neat) $v_{\max }=2932,1745,1367$, 1122, $1069 \mathrm{~cm}^{-1}$; HRMS (ESI): calcd. for $\mathrm{C}_{10} \mathrm{H}_{20} \mathrm{NaO}_{5}\left([\mathrm{M}+\mathrm{Na}]^{+}\right)$243.1203, found 243.1206.

tert-Butyl 2-(2,2-dimethoxyethoxy)propanoate (S2). Lithium diisopropylamide $\quad$ solution $\quad\left(\begin{array}{lllll}2.0 & \mathrm{M} & \text { in }\end{array}\right.$ THF/heptane/ethylbenzene, 1.1 equiv, $20.0 \mathrm{mmol}, 10 \mathrm{~mL}$ ) was added to a solution of $\mathbf{S 1}$ (1.0 equiv, $18.2 \mathrm{mmol}, 4.0 \mathrm{~g}$ ) in tetrahydrofuran $(91 \mathrm{~mL})$ at $-78^{\circ} \mathrm{C}$, and the mixture was stirred at the same temperature for $10 \mathrm{~min}$. Then, iodomethane (1.1 equiv, $20.0 \mathrm{mmol}, 1.2 \mathrm{~mL}$ ) was added to the mixture, which was allowed to slowly warm to room temperature and stirred for an additional $30 \mathrm{~min}$. The reaction was quenched with saturated aqueous $\mathrm{NH}_{4} \mathrm{Cl}$ solution, poured into water, extracted with EtOAc twice, dried over $\mathrm{MgSO}_{4}$ and then concentrated in vacuo. The crude mixture was purified by flash chromatography on silica gel (hexane/EtOAc, $\left.15: 1, \mathrm{R}_{\mathrm{f}}=0.25\right)$ to give $\mathbf{S 2}(15.37 \mathrm{mmol}, 3.6 \mathrm{~g}, 85 \%)$ as a yellow oil. ${ }^{1} \mathrm{H}$ $\operatorname{NMR}\left(\mathrm{CDCl}_{3}, 400 \mathrm{MHz}\right) \delta 4.47(\mathrm{t}, J=5.0 \mathrm{~Hz}, 1 \mathrm{H}), 3.83(\mathrm{q}, J=6.9 \mathrm{~Hz}, 1 \mathrm{H}), 3.58(\mathrm{dd}, J=10.2,5.0 \mathrm{~Hz}, 1 \mathrm{H}), 3.31-3.36$ 
(m, 7H), $1.40(\mathrm{~s}, 9 \mathrm{H}), 1.30(\mathrm{~d}, J=6.9 \mathrm{~Hz}, 3 \mathrm{H}) ;{ }^{13} \mathrm{C} \mathrm{NMR}\left(\mathrm{CDCl}_{3}, 100 \mathrm{MHz}\right) \delta 172.4,102.8,81.4,75.9,69.6,54.4,53.6$, 28.1 (3C), 18.7; IR (neat) $v_{\max }=2936,1741,1368,1119,1062,972,847 \mathrm{~cm}^{-1} ; \mathrm{HRMS}$ (ESI): calcd. for $\mathrm{C}_{11} \mathrm{H}_{22} \mathrm{NaO}_{5}([\mathrm{M}$ $+\mathrm{Na}]^{+}$) 257.1359, found 257.1355.

tert-Butyl 2-(2,2-dimethoxyethoxy)-3-(3-methoxyphenyl)-2-methylpropanoate (1). Lithium diisopropylamide solution (2.0 M in THF/heptane/ethylbenzene, 1.5 equiv, $7.7 \mathrm{mmol}, 3.8 \mathrm{~mL}$ ) was added to a solution of $\mathbf{S 2}$ (1.0 equiv, $5.1 \mathrm{mmol}, 1.2 \mathrm{~g})$ in tetrahydrofuran $(25 \mathrm{~mL})$ at $-78^{\circ} \mathrm{C}$, and the mixture was stirred at the same temperature for $20 \mathrm{~min}$. Then, 3-methoxybenzyl bromide (1.5 equiv, $7.7 \mathrm{mmol}, 1.1 \mathrm{~mL}$ ) was added to the reaction mixture, which was allowed to slowly warm to room temperature and stirred for $3 \mathrm{~h}$. The reaction was quenched with saturated aqueous $\mathrm{NH}_{4} \mathrm{Cl}$ solution, poured into water, extracted with EtOAc twice, dried over $\mathrm{MgSO}_{4}$ and then concentrated in vacuo. The crude mixture was purified by flash chromatography on silica gel (hexane/EtOAc, 7:1, $\left.\mathrm{R}_{\mathrm{f}}=0.25\right)$ to give compound 1 (5.0 mmol, $1.8 \mathrm{~g}, 97 \%)$ as a light yellow oil. ${ }^{1} \mathrm{H}$ NMR $\left(\mathrm{CDCl}_{3}, 400 \mathrm{MHz}\right) \delta 7.15(\mathrm{dd}, J=7.5,7.5 \mathrm{~Hz}, 1 \mathrm{H}), 6.80-6.84(\mathrm{~m}$, 2H), 6.71-6.76 (m, 1H), 4.51 (dd, $J=5.5,4.6 \mathrm{~Hz}, 1 \mathrm{H}), 3.77$ (s, 3H), 3.42-3.52 (m, 2H), 3.377 (s, 3H), $3.368(\mathrm{~s}, 3 \mathrm{H})$, $2.97(\mathrm{~s}, 2 \mathrm{H}), 1.40$ (s, 9H), $1.29(\mathrm{~s}, 3 \mathrm{H}) ;{ }^{13} \mathrm{C} \mathrm{NMR}\left(\mathrm{CDCl}_{3}, 100 \mathrm{MHz}\right) \delta 172.9,159.3,138.0,128.8,123.3,116.5,112.3$, 103.4, 81.7, 81.0, 64.5, 55.3, 54.10, 54.04, 45.2, 28.2 (3C), 20.7; IR (neat) $v_{\max }=2934,1723,1583,1453,1367,1259$, $1107 \mathrm{~cm}^{-1}$; HRMS (FAB): calcd. for $\mathrm{C}_{19} \mathrm{H}_{30} \mathrm{O}_{6}\left([\mathrm{M}]^{+}\right) 354.2042$, found 354.2039 .

tert-Butyl 3-(benzo[d][1,3]dioxol-5-yl)-2-(2,2-dimethoxyethoxy)-2-methylpropanoate (6a). According to the procedure of 1, compound 6a was synthesized using $1 \mathrm{mmol}$ of S2. Instead of 3-methoxybenzyl bromide, 3,4methylenedioxybenzyl bromide was used, and the reaction mixture was stirred for $5 \mathrm{~h}$. The crude mixture was purified by flash chromatography on silica gel (hexane/EtOAc, 8:1, $\left.\mathrm{R}_{\mathrm{f}}=0.25\right)$ to give $\mathbf{6 a}(254 \mathrm{mg}, 69 \%)$ as a yellow oil. ${ }^{1} \mathrm{H}$ $\operatorname{NMR}\left(\mathrm{CDCl}_{3}, 400 \mathrm{MHz}\right) \delta 6.78(\mathrm{s.} 1 \mathrm{H}), 6.67(\mathrm{~d}, J=8.4 \mathrm{~Hz}, 1 \mathrm{H}), 6.63(\mathrm{~d}, J=8.0 \mathrm{~Hz}, 1 \mathrm{H}), 5.87$ (s, 2H), $4.50(\mathrm{t}, J=5.2$ $\mathrm{Hz}, 1 \mathrm{H}), 3.41-3.49$ (m, 2H), 3.37 (s, 3H), 3.37 (s, 3H), 2.89 (s, 2H), 1.39 (s, 9H), $1.26(\mathrm{~s}, 3 \mathrm{H}) ;{ }^{13} \mathrm{C} \mathrm{NMR}\left(\mathrm{CDCl}_{3}, 100\right.$ MHz) $\delta 172.8,147.2,146.3,130.1,123.8,111.3,107.7,103.4,100.9,81.6,81.0,64.5,54.2,54.0,44.9,28.1$ (3C), 20.6; IR (neat) $v_{\max }=2933,1732,1489,1442,1368,1243,1107,1038 \mathrm{~cm}^{-1}$; HRMS (FAB): calcd. for $\mathrm{C}_{19} \mathrm{H}_{28} \mathrm{O}_{7}\left([\mathrm{M}]^{+}\right)$ 368.1835 , found 368.1835 .

tert-Butyl 2-(2,2-dimethoxyethoxy)-3-(3,5-dimethoxyphenyl)-2-methylpropanoate (6b). According to the procedure of $\mathbf{1}$, compound $\mathbf{6} \mathbf{b}$ was synthesized using $1 \mathrm{mmol}$ of $\mathbf{S 2}$. Instead of 3-methoxybenzyl bromide, 3,5-dimethoxybenzyl bromide was used, and the reaction mixture was stirred for $13 \mathrm{~h}$. The crude mixture was purified by flash chromatography on silica gel (hexane/EtOAc, $\left.6: 1, \mathrm{R}_{\mathrm{f}}=0.25\right)$ to give $\mathbf{6 b}(292 \mathrm{mg}, 76 \%)$ as a yellow oil. ${ }^{1} \mathrm{H} \mathrm{NMR}\left(\mathrm{CDCl}_{3}\right.$, $400 \mathrm{MHz}) \delta 6.44(\mathrm{~d}, J=2.3 \mathrm{~Hz}, 2 \mathrm{H}), 6.33(\mathrm{dd}, J=2.3,2.3 \mathrm{~Hz}, 1 \mathrm{H}), 4.53(\mathrm{dd}, J=5.8,5.0 \mathrm{~Hz}, 1 \mathrm{H}), 3.74(\mathrm{~s}, 6 \mathrm{H}), 3.47$ $(\mathrm{m}, 2 \mathrm{H}), 3.383(\mathrm{~s}, 3 \mathrm{H}), 3.338(\mathrm{~s}, 3 \mathrm{H}), 2.93$ (d, J=3.2 Hz, 2H), 1.43 (s, 9H), 1.31 (s, 3H); ${ }^{13} \mathrm{C} \mathrm{NMR}\left(\mathrm{CDCl}_{3}, 100 \mathrm{MHz}\right)$ $\delta$ 172.8, 160.3 (2C), 138.6, 108.9 (2C), 103.2, 98.8, 81.5, 80.9, 64.4, 55.3 (2C), 53.9 (2C), 45.3, 28.1 (3C), 20.6; IR (neat) $v_{\max }=2934,1725,1595,1459,1202,1149,1108,1058 \mathrm{~cm}^{-1}$; HRMS (FAB): calcd. for $\mathrm{C}_{20} \mathrm{H}_{32} \mathrm{O}_{7}\left([\mathrm{M}]^{+}\right) 384.2148$, found 384.2145 .

tert-Butyl 2-(2,2-dimethoxyethoxy)-3-(4-fluoro-3-methoxyphenyl)-2-methylpropanoate (6c). According to the procedure of 1, compound $\mathbf{6 c}$ was synthesized using $1 \mathrm{mmol}$ of $\mathbf{S 2}$. Instead of 3-methoxybenzyl bromide, 3-methoxy4-fluorobenzyl bromide was used, and the reaction mixture was stirred for $15 \mathrm{~h}$. The crude mixture was purified by flash chromatography on silica gel (hexane/EtOAc, $\left.6: 1, \mathrm{R}_{\mathrm{f}}=0.25\right)$ to give $\mathbf{6 c}(208 \mathrm{mg}, 56 \%)$ as a colorless oil. ${ }^{1} \mathrm{H}$ NMR $\left(\mathrm{CDCl}_{3}, 400 \mathrm{MHz}\right) \delta 6.87-7.05(\mathrm{~m}, 2 \mathrm{H}), 6.69-6.77(\mathrm{~m}, 1 \mathrm{H}), 4.50-4.55(\mathrm{~m}, 1 \mathrm{H}), 3.87(\mathrm{~s}, 3 \mathrm{H}), 3.43-3.60(\mathrm{~m}, 2 \mathrm{H}), 3.38$ 
(s, 3H), 3.37 (s, 3H), 2.87-3.04 (m, 2H), $1.41(\mathrm{~s}, 9 \mathrm{H}), 1.31$ (s, 3H); $\left.{ }^{13} \mathrm{C} \mathrm{NMR} \mathrm{(CDCl} 3,100 \mathrm{MHz}\right) \delta 172.8,137.3,136.4$, $131.7,127.9,127.8,127.4,103.4,81.5,81.1,64.6,54.2,54.0,45.2,28.1$ (3C), 21.5, 20.7; IR (neat) $v_{\max }=2978,1730$, 1609, 1517, 1461, $1118 \mathrm{~cm}^{-1}$; HRMS (FAB): calcd. for $\mathrm{C}_{19} \mathrm{H}_{30} \mathrm{FO}_{6}\left([\mathrm{M}+\mathrm{H}]^{+}\right)$373.2002, found 373.2005.

tert-Butyl 2-(2,2-dimethoxyethoxy)-2-methyl-3-(m-tolyl)propanoate (6d). According to the procedure of 1, compound 6d was synthesized using $1 \mathrm{mmol}$ of $\mathbf{S 2}$. Instead of 3-methoxybenzyl bromide, 3-methylbenzyl bromide was used, and the reaction mixture was stirred for $15 \mathrm{~h}$. The crude mixture was purified by flash chromatography on silica gel (hexane/EtOAc, $\left.7: 1, \mathrm{R}_{\mathrm{f}}=0.25\right)$ to give $6 \mathrm{c}(257 \mathrm{mg}, 76 \%)$ as an ivory oil. ${ }^{1} \mathrm{H} \mathrm{NMR}\left(\mathrm{CDCl}_{3}, 400 \mathrm{MHz}\right) \delta 7.12(\mathrm{~d}, J$ $=7.6 \mathrm{~Hz}, 1 \mathrm{H}), 6.99-7.05(\mathrm{~m}, 3 \mathrm{H}), 4.51(\mathrm{t}, J=5.2 \mathrm{~Hz}, 1 \mathrm{H}), 3.44-3.51(\mathrm{~m}, 2 \mathrm{H}), 3.382(\mathrm{~s}, 3 \mathrm{H}), 3.379(\mathrm{~s}, 3 \mathrm{H}), 2.96(\mathrm{~s}, 2 \mathrm{H})$, $2.29(\mathrm{~s}, 3 \mathrm{H}), 1.40(\mathrm{~s}, 9 \mathrm{H}), 1.29(\mathrm{~s}, 3 \mathrm{H}) ;{ }^{13} \mathrm{C} \mathrm{NMR}\left(\mathrm{CDCl}_{3}, 400 \mathrm{MHz}\right) \delta 172.8,137.3,136.4,131.7,127.9,127.8,127.4$, 103.4, 81.5, 81.1, 64.6, 54.2, 54.0, 45.2, 28.1 (3C), 21.5, 20.7; IR (neat) $v_{\max }=2930,1725,1454,1368,1099 \mathrm{~cm}^{-1}$; HRMS (ESI): calcd. for $\mathrm{C}_{19} \mathrm{H}_{30} \mathrm{NaO}_{5}\left([\mathrm{M}+\mathrm{Na}]^{+}\right)$361.1985, found 361.1977 .

tert-Butyl 2-(2,2-dimethoxyethoxy)-2-methyl-3-phenylpropanoate (6e). According to the procedure of 1 , compound 6e was synthesized using $1 \mathrm{mmol}$ of $\mathbf{S 2}$. Instead of 3-methoxybenzyl bromide, benzyl bromide was used, and the reaction mixture was stirred for $7 \mathrm{~h}$. The crude mixture was purified by flash chromatography on silica gel (hexane/EtOAc, 8:1, $\left.\mathrm{R}_{\mathrm{f}}=0.25\right)$ to give $6 \mathrm{e}(220 \mathrm{mg}, 68 \%)$ as a light yellow oil; ${ }^{1} \mathrm{H}$ NMR $\left(\mathrm{CDCl}_{3}, 400 \mathrm{MHz}\right) \delta 7.19-7.26(\mathrm{~m}, 5 \mathrm{H}), 4.52(\mathrm{t}, J$ $=5.3 \mathrm{~Hz}, 1 \mathrm{H}), 3.48(\mathrm{~m}, 2 \mathrm{H}), 3.392(\mathrm{~s}, 3 \mathrm{H}), 3.384(\mathrm{~s}, 3 \mathrm{H}), 3.01(\mathrm{~s}, 2 \mathrm{H}), 1.41(\mathrm{~s}, 9 \mathrm{H}), 1.31(\mathrm{~s}, 3 \mathrm{H}) ;{ }^{13} \mathrm{C} \mathrm{NMR}\left(\mathrm{CDCl}_{3}\right.$, $100 \mathrm{MHz}) \delta 172.9,136.5,130.9$ (2C), 128.0 (2C), 126.7, 103.4, 81.6, 81.0, 64.6, 54.2, 54.1, 45.3, 28.2 (3C), 20.8; IR (neat) $v_{\max }=2933,1725,1368,1107 \mathrm{~cm}^{-1}$; HRMS (ESI): calcd. for $\mathrm{C}_{18} \mathrm{H}_{28} \mathrm{NaO}_{5}\left([\mathrm{M}+\mathrm{Na}]^{+}\right) 347.1829$, found 347.1826.

tert-Butyl 2-(2,2-dimethoxyethoxy)-2-methyl-3-(naphthalen-2-yl)propanoate (6f). According to the procedure of 1 , compound $\mathbf{6 f}$ was synthesized using $1 \mathrm{mmol}$ of $\mathbf{S 2}$. Instead of 3-methoxybenzyl bromide, 2-(bromomethyl)naphthalene was used, and the reaction mixture was stirred for $16 \mathrm{~h}$. The crude mixture was purified by flash chromatography on silica gel (hexane/EtOAc, $\left.8: 1, \mathrm{R}_{\mathrm{f}}=0.3\right)$ to give $\mathbf{6 f}(261 \mathrm{mg}, 68 \%)$ as a colorless oil; ${ }^{1} \mathrm{H}$ NMR $\left(\mathrm{CDCl}_{3}, 400 \mathrm{MHz}\right) \delta 7.70$ $7.82(\mathrm{~m}, 4 \mathrm{H}), 7.42-7.47(\mathrm{~m}, 3 \mathrm{H}), 4.57(\mathrm{t}, J=5.3 \mathrm{~Hz}, 1 \mathrm{H}), 3.57(\mathrm{dd}, J=9.7,4.9 \mathrm{~Hz}, 1 \mathrm{H}), 3.51(\mathrm{dd}, J=9.7,5.7 \mathrm{~Hz}, 1 \mathrm{H})$, $3.42(\mathrm{~s}, 3 \mathrm{H}), 3.41(\mathrm{~s}, 3 \mathrm{H}), 3.20(\mathrm{~s}, 2 \mathrm{H}), 1.42(\mathrm{~s}, 9 \mathrm{H}), 1.37(\mathrm{~s}, 3 \mathrm{H}) ;{ }^{13} \mathrm{C} \mathrm{NMR}\left(\mathrm{CDCl}_{3}, 100 \mathrm{MHz}\right) \delta 172.8,134.2,133.3$, 132.5, 129.4, 129.4, 127.7, 127.7, 127.3, 125.9, 125.5, 103.4, 81.6, 81.1, 64.5, 54.1, 54.0, 45.4, 28.1 (3C), 20.7; IR (neat) $v_{\max }=2932,1724,1367,1162,1106 \mathrm{~cm}^{-1}$; HRMS (FAB): calcd. for $\mathrm{C}_{22} \mathrm{H}_{30} \mathrm{O}_{5}\left([\mathrm{M}]^{+}\right)$374.2093, found 374.2090.

tert-Butyl 3-(3-bromophenyl)-2-(2,2-dimethoxyethoxy)-2-methylpropanoate (6g). According to the procedure of $\mathbf{1}$, compound $\mathbf{6 g}$ was synthesized using $1 \mathrm{mmol}$ of $\mathbf{S 2}$. Instead of 3-methoxybenzyl bromide, 3-bromobenzyl bromide was used, and the reaction mixture was stirred for $4 \mathrm{~h}$. The crude mixture was purified by flash chromatography on silica gel (hexane/EtOAc, 7:1, $\left.\mathrm{R}_{\mathrm{f}}=0.25\right)$ to give $\mathbf{6 g}(92 \mathrm{mg}, 23 \%)$ as a light yellow oil; ${ }^{1} \mathrm{H} \mathrm{NMR}\left(\mathrm{CDCl}_{3}, 400 \mathrm{MHz}\right) \delta 7.46(\mathrm{t}, J$ $=1.8 \mathrm{~Hz}, 1 \mathrm{H}), 7.34(\mathrm{ddd}, J=7.8,2.1,1.3 \mathrm{~Hz}, 1 \mathrm{H}), 7.18(\mathrm{dt}, J=7.7,1.4 \mathrm{~Hz}, 1 \mathrm{H}), 7.11(\mathrm{t}, J=7.7 \mathrm{~Hz}, 1 \mathrm{H}), 4.52(\mathrm{dd}, J=$ 5.8, 4.8 Hz, 1H), 3.43-3.55 (m, 2H), $3.41(\mathrm{~s}, 3 \mathrm{H}), 3.40(\mathrm{~s}, 3 \mathrm{H}), 2.96(\mathrm{~d}, J=1.9 \mathrm{~Hz}, 2 \mathrm{H}), 1.41(\mathrm{~s}, 9 \mathrm{H}), 1.32(\mathrm{~s}, 3 \mathrm{H}) ;{ }^{13} \mathrm{C}$ NMR $\left(\mathrm{CDCl}_{3}, 100 \mathrm{MHz}\right) \delta 172.5,138.9,133.8,129.8,129.5,129.4,122.0,103.4$, 81.9, 80.7, 64.5, 54.3, 54.1, 44.9, 28.1 (3C), 20.8; IR (neat) $v_{\max } 2928,1728,1369,1112=\mathrm{cm}^{-1}$; HRMS (CI): calcd. for $\mathrm{C}_{18} \mathrm{H}_{28} \mathrm{BrO}_{5}\left([\mathrm{M}+\mathrm{H}]^{+}\right) 403.1120$, found 403.1111 .

tert-Butyl 2-(2,2-dimethoxyethoxy)-2-methyl-3-(1-tosyl-1H-indol-2-yl)propanoate

(6h). Lithium bis(trimethylsilyl)amide solution (1.0 M in THF, 1.5 equiv., $1.83 \mathrm{mmol}, 1.8 \mathrm{~mL})$ was added to a solution of $\mathbf{S 2}(1.0$ equiv, $1.22 \mathrm{mmol}, 286 \mathrm{mg})$ in tetrahydrofuran $(8 \mathrm{~mL})$ at $-78^{\circ} \mathrm{C}$, and the mixture was stirred at the same temperature for 
$20 \mathrm{~min}$. Then, a solution of 2-(bromomethyl)-1-tosyl- $1 H$-indole ${ }^{8}$ (1.3 equiv., $1.6 \mathrm{mmol}, 578 \mathrm{mg}$ ) in tetrahydrofuran (4 $\mathrm{mL}$ ) was added to the reaction mixture, which was allowed to slowly warm to room temperature and stirred for $14 \mathrm{~h}$. The reaction was quenched with saturated aqueous $\mathrm{NH}_{4} \mathrm{Cl}$ solution, poured into water, extracted with EtOAc twice, dried over $\mathrm{Na}_{2} \mathrm{SO}_{4}$ and then concentrated in vacuo. The crude mixture was purified by flash chromatography on silica gel (hexane/EtOAc, $\left.9: 1, \mathrm{R}_{\mathrm{f}}=0.25\right)$ to give $\mathbf{6 h}(0.65 \mathrm{mmol}, 335 \mathrm{mg}, 53 \%)$ as a yellow oil. ${ }^{1} \mathrm{H} \mathrm{NMR}\left(\mathrm{CDCl}_{3}, 400 \mathrm{MHz}\right)$ $\delta 8.15(\mathrm{~d}, J=8.4 \mathrm{~Hz}, 1 \mathrm{H}), 7.55(\mathrm{~d}, J=8.4 \mathrm{~Hz}, 2 \mathrm{H}), 7.38(\mathrm{~d}, J=7.6 \mathrm{~Hz}, 1 \mathrm{H}), 7.12-7.25(\mathrm{~m}, 4 \mathrm{H}), 6.75(\mathrm{~s}, 1 \mathrm{H}), 4.53(\mathrm{t}, J$ $=5.2 \mathrm{~Hz}, 1 \mathrm{H}), 3.55-3.60(\mathrm{~m}, 3 \mathrm{H}), 3.44-3.49(\mathrm{~m}, 1 \mathrm{H}), 3.38(\mathrm{~s}, 3 \mathrm{H}), 3.35(\mathrm{~s}, 3 \mathrm{H}), 2.29(\mathrm{~s}, 3 \mathrm{H}), 1.44(\mathrm{~s}, 3 \mathrm{H}), 1.37(\mathrm{~s}, 9 \mathrm{H})$; ${ }^{13} \mathrm{C} \mathrm{NMR}\left(\mathrm{CDCl}_{3}, 100 \mathrm{MHz}\right) \delta 172.5,144.9,137.1,136.4,136.1,130.3,129.9$ (2C), 126.5 (2C), 124.3, 123.8, 120.6, $115.3,112.5,103.4,81.9,80.2,64.4,54.3,54.2,36.1,28.1$ (3C), 21.8, 21.7; IR (neat) $v_{\max }=2930,1728,1450,1367$, 1173, $1089 \mathrm{~cm}^{-1}$; HRMS (FAB): calcd. for $\mathrm{C}_{27} \mathrm{H}_{35} \mathrm{NO}_{7} \mathrm{~S}\left([\mathrm{M}]^{+}\right)$517.2134, found 517.2128.

tert-Butyl 2-(2,2-dimethoxyethoxy)-2-methyl-3-(thiophen-2-yl)propanoate (6i). According to the procedure of 1, compound 6i was synthesized using $1 \mathrm{mmol}$ of $\mathbf{S 2}$. Instead of 3-methoxybenzyl bromide, 3-bromomethylthiophene was used, and the reaction mixture was stirred for $7 \mathrm{~h}$. The crude mixture was purified by flash chromatography on silica gel (hexane/EtOAc, 8:1, $\left.\mathrm{R}_{\mathrm{f}}=0.25\right)$ to give $\mathbf{6 i}(247 \mathrm{mg}, 75 \%)$ as a colorless oil; ${ }^{1} \mathrm{H}$ NMR $\left(\mathrm{CDCl}_{3}, 400 \mathrm{MHz}\right) \delta 7.16(\mathrm{dd}, J=$ $5.1,1.2 \mathrm{~Hz}, 1 \mathrm{H}), 6.91(\mathrm{dd}, J=5.1,3.4 \mathrm{~Hz}, 1 \mathrm{H}), 6.86(\mathrm{dd}, J=3.4,1.2 \mathrm{~Hz}, 1 \mathrm{H}), 4.57$ (dd, $J=5.6,4.8 \mathrm{~Hz}, 1 \mathrm{H}), 3.58$ (dd, $J=9.6,4.8 \mathrm{~Hz}, 1 \mathrm{H}), 3.50(\mathrm{dd}, J=9.7,5.7 \mathrm{~Hz}, 1 \mathrm{H}), 3.42$ (s, 3H), $3.41(\mathrm{~s}, 3 \mathrm{H}), 3.23$ (d, $J=0.9 \mathrm{~Hz}, 2 \mathrm{H}), 1.43(\mathrm{~s}, 9 \mathrm{H})$, 1.35 (s, 3H); ${ }^{13} \mathrm{C} \mathrm{NMR}\left(\mathrm{CDCl}_{3}, 100 \mathrm{MHz}\right) \delta 172.5,138.0,127.5,126.3,125.0,103.4,81.8,80.5,64.8,54.3,54.1,39.3$, 28.1 (3C), 20.8; IR (neat) $v_{\max }=2932,1728,1369,1252,1112 \mathrm{~cm}^{-1}$; HRMS (CI): calcd. for $\mathrm{C}_{16} \mathrm{H}_{25} \mathrm{O}_{5} \mathrm{~S}\left([\mathrm{M}-\mathrm{H}]^{+}\right)$ 329.1423, found 329.1423 .

\section{tert-Butyl 3-(benzo[b]thiophen-2-yl)-2-(2,2-dimethoxyethoxy)-2-methylpropanoate $(6 \mathbf{6})$. According to the} procedure of $\mathbf{1}$, compound $\mathbf{6 j}$ was synthesized using $1 \mathrm{mmol}$ of $\mathbf{S 2}$. Instead of 3-methoxybenzyl bromide, 2(bromomethyl)benzo[ $b]$ thiophene ${ }^{9}$ was used, and the reaction mixture was stirred for $1 \mathrm{~h}$ at $0{ }^{\circ} \mathrm{C}$. The crude mixture was purified by flash chromatography on silica gel (hexane/EtOAc, 10:1, $\left.\mathrm{R}_{\mathrm{f}}=0.2\right)$ to give $\mathbf{6 j}(285 \mathrm{mg}, 75 \%$ ) as a colorless oil. ${ }^{1} \mathrm{H}$ NMR $\left(\mathrm{CD}_{3} \mathrm{OD}, 400 \mathrm{MHz}\right) \delta 7.75-7.77(\mathrm{~m}, 1 \mathrm{H}), 7.68$ (dd, $\left.J=7.1,1.6 \mathrm{~Hz}, 1 \mathrm{H}\right), 7.22-7.30$ (m, 2H), 7.14 (s, 1H), 4.59 (t, $J=5.3 \mathrm{~Hz}, 1 \mathrm{H}), 3.59$ (dd, $J=9.6,5.2 \mathrm{~Hz}, 1 \mathrm{H}), 3.52(\mathrm{dd}, J=10.0,5.2 \mathrm{~Hz}, 1 \mathrm{H}), 3.42(\mathrm{~s}, 3 \mathrm{H}), 3.41$ (s, $3 \mathrm{H}), 3.29$ (br s, 2H), 1.41 (s, 9H), 1.40 (s, 3H); ${ }^{13} \mathrm{C} \mathrm{NMR}\left(\mathrm{CD}_{3} \mathrm{OD}, 100 \mathrm{MHz}\right) \delta 173.8,141.9,141.1,140.5,125.4,125.2$, 125.0, 124.0, 122.9, 104.9, 83.2, 81.6, 65.8, 54.8, 54.6, 41.0, 28.3 (3C), 21.4; IR (neat) $v_{\max }=2978,1725,1368,1253$, $1109 \mathrm{~cm}^{-1}$; HRMS (FAB): calcd. for $\mathrm{C}_{20} \mathrm{H}_{28} \mathrm{O}_{5} \mathrm{~S}\left([\mathrm{M}]^{+}\right) 380.1657$, found 380.1657 .

tert-Butyl 3-(benzofuran-2-yl)-2-(2,2-dimethoxyethoxy)-2-methylpropanoate (6k). According to the procedure of 1, compound $\mathbf{6 k}$ was synthesized using $1 \mathrm{mmol}$ of $\mathbf{S 2}$. Instead of 3-methoxybenzyl bromide, 2-(bromomethyl)-1benzofuran was used, and the reaction mixture was stirred for $1 \mathrm{~h}$ at $0{ }^{\circ} \mathrm{C}$. The crude mixture was purified by flash chromatography on silica gel (hexane/EtOAc, $\left.10: 1, \mathrm{R}_{\mathrm{f}}=0.2\right)$ to give $\mathbf{6 k}(313 \mathrm{mg}, 83 \%)$ as a colorless oil. ${ }^{1} \mathrm{H}$ NMR $\left(\mathrm{CDCl}_{3}, 400 \mathrm{MHz}\right) \delta 7.47-7.51(\mathrm{~m}, 1 \mathrm{H}), 7.38(\mathrm{~d}, J=7.68 \mathrm{~Hz}, 1 \mathrm{H}), 7.13-7.23(\mathrm{~m}, 2 \mathrm{H}), 6.54(\mathrm{~s}, 1 \mathrm{H}), 4.53(\mathrm{t}, J=5.2 \mathrm{~Hz}$, $1 \mathrm{H}), 3.58(\mathrm{dd}, J=9.6,4.9 \mathrm{~Hz}, 1 \mathrm{H}), 3.52(\mathrm{dd}, J=9.6,5.6 \mathrm{~Hz}, 1 \mathrm{H}), 3.40(\mathrm{~s}, 3 \mathrm{H}), 3.39(\mathrm{~s}, 3 \mathrm{H}), 3.23(\mathrm{q}, J=8.0 \mathrm{~Hz}, 2 \mathrm{H})$,

\footnotetext{
${ }^{8}$ For the synthetic procedure of 2-(bromomethyl)-1-tosyl-1H-indole, see: Chem. Commun. 2014, 50, 8904-8907. $N$-Bromosuccinimide was used instead of $N$-chlorosuccinimide. $65 \%$ yield; White solid; $\mathrm{mp} 71{ }^{\circ} \mathrm{C} ;{ }^{1} \mathrm{H}$ NMR $\left(\mathrm{CDCl}_{3}, 400 \mathrm{MHz}\right) \delta 8.09(\mathrm{~d}, J=8.2 \mathrm{~Hz}, 1 \mathrm{H}), 7.81(\mathrm{~d}, J=8.2 \mathrm{~Hz}$, $2 \mathrm{H}), 7.50(\mathrm{~d}, J=7.8 \mathrm{~Hz}, 1 \mathrm{H}), 7.33-7.37(\mathrm{~m}, 1 \mathrm{H}), 7.22-7.28(\mathrm{~m}, 3 \mathrm{H}), 6.83(\mathrm{~s}, 1 \mathrm{H}), 5.04(\mathrm{~s}, 2 \mathrm{H}), 2.36(\mathrm{~s}, 3 \mathrm{H}) ;{ }^{13} \mathrm{C} \mathrm{NMR}\left(\mathrm{CDCl}_{3}, 100 \mathrm{MHz}\right) \delta 145.3$, $137.5,136.7,135.7,130.0(2 \mathrm{C}), 128.9,127.0(2 \mathrm{C}), 125.8,124.1,121.4,115.0,114.0,25.2,21.8$; IR (neat) $v_{\max }=1446,1369,1298,1250,1170$, $1150 \mathrm{~cm}^{-1}$; HRMS(FAB): calcd. for $\mathrm{C}_{16} \mathrm{H}_{14} \mathrm{BrNO}_{2} \mathrm{~S}\left([\mathrm{M}]^{+}\right) 362.9929$, found 362.9933 .

${ }^{9}$ For the synthetic procedure of 2-(bromomethyl)benzo[b]thiophene, see: ACS Med. Chem. Lett. 2011, 2, 919-923. ${ }^{1} \mathrm{H} \mathrm{NMR}\left(\mathrm{CDCl}_{3}, 400 \mathrm{MHz}\right)$ 7.76-7.78 (m, 1H), 7.69-7.72 (m, 1H), 7.30-7.34 (m, 2H), $7.31(\mathrm{~s}, 1 \mathrm{H}), 4.78(\mathrm{~s}, 2 \mathrm{H})$.
} 
1.46 (s, 9H), 1.44 (s, 3H); ${ }^{13} \mathrm{C} \mathrm{NMR}\left(\mathrm{CDCl}_{3}, 100 \mathrm{MHz}\right) \delta 172.1,154.8,154.1,128.9,123.6,122.6,120.6,110.9,105.5$, 103.3, 81.9, 80.0, 64.7, 54.3, 54.0, 37.4, 28.1 (3C), 21.4.; IR (neat) $v_{\max }=2935,1729,1454,1369,1253,1109 \mathrm{~cm}^{-1}$; HRMS (FAB): calcd. for $\mathrm{C}_{20} \mathrm{H}_{28} \mathrm{O}_{6}\left([\mathrm{M}]^{+}\right)$364.1886, found 364.1880.
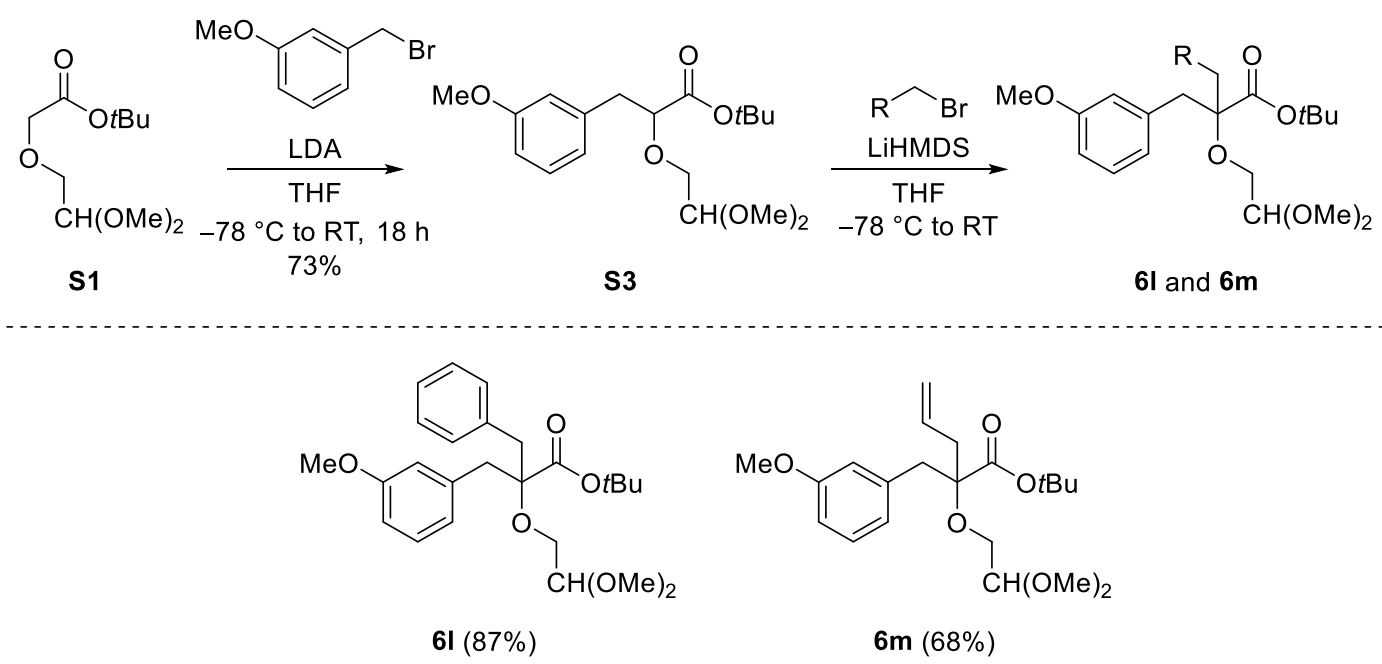

Scheme S2. Synthesis of Friedel-Crafts substrates $6 \mathbf{l}$ and $\mathbf{6 m}$

tert-Butyl 2-(2,2-dimethoxyethoxy)-3-(3-methoxyphenyl)propanoate (S3). Lithium diisopropylamide solution (2.0 $\mathrm{M}$ in THF/heptane/ethylbenzene, 1.1 equiv, $15.0 \mathrm{mmol}, 7.5 \mathrm{~mL}$ ) was added to a solution of compound $\mathbf{S 1}$ (1 equiv, 13.6 mmol, $3.0 \mathrm{~g})$ in tetrahydrofuran $(68 \mathrm{~mL})$ at $-78{ }^{\circ} \mathrm{C}$, and the mixture was stirred at the same temperature for $10 \mathrm{~min}$. Then, 3-methoxybenzyl bromide (1.1 equiv, $15.0 \mathrm{mmol}, 2.1 \mathrm{~mL}$ ) was added to the reaction mixture, which was allowed to slowly warm to room temperature and stirred for $18 \mathrm{~h}$. The reaction was quenched with saturated aqueous $\mathrm{NH}_{4} \mathrm{Cl}$ solution, poured into water, extracted with EtOAc twice, dried over $\mathrm{MgSO}_{4}$ and then concentrated in vacuo. The crude mixture was purified by flash chromatography on silica gel (hexane/EtOAc, 10:1, $\mathrm{R}_{\mathrm{f}}=0.2$ ) to give compound $\mathbf{S 3}$ (9.9 mmol, $3.4 \mathrm{~g}, 73 \%)$ as a light yellow oil. ${ }^{1} \mathrm{H} \mathrm{NMR}\left(\mathrm{CDCl}_{3}, 400 \mathrm{MHz}\right) \delta 7.16(\mathrm{dd}, J=8.0,8.0 \mathrm{~Hz}, 1 \mathrm{H}), 6.80-6.83(\mathrm{~m}$, 2H), $6.73(\mathrm{dd}, J=8.4,2.4 \mathrm{~Hz}, 1 \mathrm{H}), 4.41(\mathrm{t}, J=5.0 \mathrm{~Hz}, 1 \mathrm{H}), 3.97(\mathrm{dd}, J=7.8,5.4 \mathrm{~Hz}, 1 \mathrm{H}), 3.76(\mathrm{~s}, 3 \mathrm{H}), 3.60(\mathrm{dd}, J=$ 10.4, $4.8 \mathrm{~Hz}, 1 \mathrm{H}), 3.29-3.34(\mathrm{~m}, 1 \mathrm{H}), 3.31(\mathrm{~s}, 3 \mathrm{H}), 3.24(\mathrm{~s}, 3 \mathrm{H}), 2.90-3.00(\mathrm{~m}, 2 \mathrm{H}), 1.40(\mathrm{~s}, 9 \mathrm{H}) ;{ }^{13} \mathrm{C} \mathrm{NMR}\left(\mathrm{CDCl}_{3}\right.$, $100 \mathrm{MHz}) \delta 171.3,159.6,139.0,129.3,122.0,115.2,112.3,102.9,81.8,81.1,70.2,55.3,54.2,53.8,39.4,28.2$ (3C); IR (neat) $v_{\max }=2933,1738,1601,1454,1367,1256,1151,1141,1043 \mathrm{~cm}^{-1}$; HRMS (FAB): calcd. for $\mathrm{C}_{18} \mathrm{H}_{28} \mathrm{O}_{6}\left([\mathrm{M}]^{+}\right)$ 340.1886 , found 340.1881 .

\section{tert-Butyl 2-benzyl-2-(2,2-dimethoxyethoxy)-3-(3-methoxyphenyl)propanoate}

(61).

Lithium

bis(trimethylsilyl)amide solution (1.0 M in THF, 1.5 equiv., $1.3 \mathrm{mmol}, 1.3 \mathrm{~mL}$ ) was added to a solution of compound S3 (1 equiv, $0.88 \mathrm{mmol}, 300 \mathrm{mg}$ ) in tetrahydrofuran $\left(9 \mathrm{~mL}\right.$ ) at $-78{ }^{\circ} \mathrm{C}$, and the mixture was stirred at the same temperature for $20 \mathrm{~min}$. Then, benzyl bromide (1.5 equiv, $1.3 \mathrm{mmol}, 157 \mu \mathrm{L}$ ) was added to the reaction mixture, which was allowed to slowly warm to room temperature and stirred for $2 \mathrm{~h}$. The reaction was quenched with saturated aqueous $\mathrm{NH}_{4} \mathrm{Cl}$ solution, poured into water, extracted with EtOAc twice, dried over $\mathrm{MgSO}_{4}$ and then concentrated in vacuo. The crude mixture was purified by flash chromatography on silica gel (hexane/EtOAc, 10:1, $R_{f}=0.25$ ) to give compound 61 $(0.77 \mathrm{mmol}, 330 \mathrm{mg}, 87 \%)$ as a light yellow oil. ${ }^{1} \mathrm{H} \mathrm{NMR}\left(\mathrm{CDCl}_{3}, 400 \mathrm{MHz}\right) \delta 7.19-7.27(\mathrm{~m}, 5 \mathrm{H}), 7.15(\mathrm{dd}, J=8.0$, $8.0 \mathrm{~Hz}, 1 \mathrm{H}), 6.83-6.86(\mathrm{~m}, 2 \mathrm{H}), 6.75(\mathrm{dd}, J=8.0,2.0 \mathrm{~Hz}, 1 \mathrm{H}), 4.59(\mathrm{t}, J=5.2 \mathrm{~Hz}, 1 \mathrm{H}), 3.76(\mathrm{~s}, 3 \mathrm{H}), 3.69$ (d, $J=5.6$

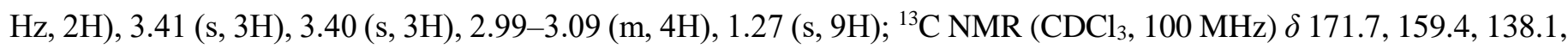
136.6, 130.7 (2C), 129.0, 128.1 (2C), 126.7, 123.1, 116.2, 112.4, 103.5, 84.1, 82.0, 64.8, 55.3, 54.3, 54.2, 41.2, 41.1, 
28.0 (3C); IR (neat) $v_{\max }=2932,1717,1584,1453,1367,1259,1152 \mathrm{~cm}^{-1}$; HRMS (FAB): calcd. for $\mathrm{C}_{25} \mathrm{H}_{34} \mathrm{O}_{6}\left([\mathrm{M}]^{+}\right)$ 430.2355 , found 430.2352 .

tert-Butyl 2-(2,2-dimethoxyethoxy)-2-(3-methoxybenzyl)pent-4-enoate $(6 \mathrm{~m})$. According to the procedure of 61 , compound $\mathbf{6 m}$ was synthesized using $1 \mathrm{mmol}$ of $\mathbf{S 3}$. Instead of benzyl bromide, allyl bromide was used, and the reaction mixture was stirred for $14 \mathrm{~h}$. The crude mixture was purified by flash chromatography on silica gel (hexane/EtOAc, 7:1, $\left.\mathrm{R}_{\mathrm{f}}=0.3\right)$ to give $6 \mathrm{~m}(258 \mathrm{mg}, 68 \%)$ as a yellow oil. ${ }^{1} \mathrm{H}$ NMR $\left(\mathrm{CD}_{3} \mathrm{OD}, 400 \mathrm{MHz}\right) \delta 7.13(\mathrm{dd}, J=7.8,7.8 \mathrm{~Hz}, 1 \mathrm{H}), 6.87$ (br s, 1H), $6.82(\mathrm{~d}, J=7.8 \mathrm{~Hz}, 1 \mathrm{H}), 6.76$ (dd, $J=8.3,2.8 \mathrm{~Hz}, 1 \mathrm{H}), 5.82-5.94$ (m, 1H), 5.17-5.19 (m, 1H), 5.14 (br s, 1H), 4.53 (t, $J=5.1 \mathrm{~Hz}, 1 \mathrm{H}), 3.75$ (d, $J=4.6 \mathrm{~Hz}, 3 \mathrm{H}), 3.57$ (AB q, $J=9.6 \mathrm{~Hz}, 1 \mathrm{H}), 3.56$ (AB q, $J=8.0 \mathrm{~Hz}, 1 \mathrm{H}), 3.38$ (s, 3H), 3.37 (s, 3H), $2.98(\mathrm{AB} \mathrm{q}, J=14.0 \mathrm{~Hz}, 2 \mathrm{H}), 2.56(\mathrm{qd}, J=14.9,7.2 \mathrm{~Hz}, 2 \mathrm{H}), 1.36(\mathrm{~s}, 9 \mathrm{H}) ;{ }^{13} \mathrm{C}$ NMR $\left(\mathrm{CD}_{3} \mathrm{OD}\right.$, $100 \mathrm{MHz}) \delta 173.1,160.9,139.2,134.0,129.9,124.1,119.3,117.4,113.2,104.9,84.2,83.2,65.4,55.7,54.7,54.6,42.4$, 39.8, 28.4 (3C); IR (neat) $v_{\max }=2933,1724,1559,1439,1368,1259,1152 \mathrm{~cm}^{-1}$; HRMS (FAB): calcd. for $\mathrm{C}_{21} \mathrm{H}_{32} \mathrm{O}_{6}$ $\left([\mathrm{M}]^{+}\right) 380.2199$, found 380.2196 .

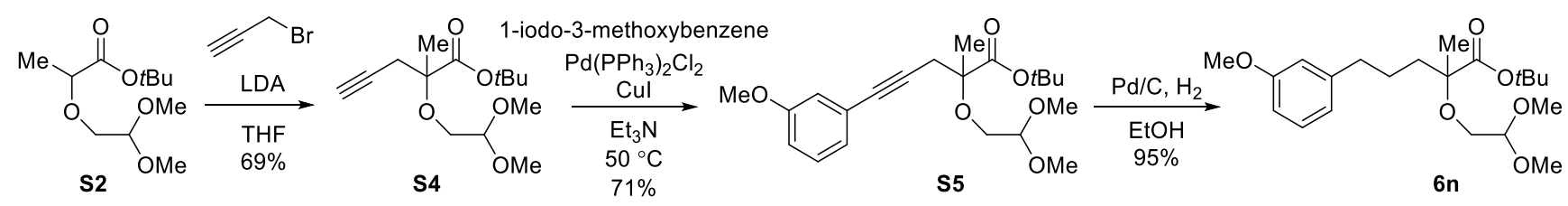

Scheme S3. Synthesis of Friedel-Crafts substrates 6n

tert-Butyl 2-(2,2-dimethoxyethoxy)-2-methylpent-4-ynoate (S4). Lithium diisopropylamide solution (2.0 M in THF/heptane/ethylbenzene, 1.1 equiv, $1.98 \mathrm{mmol}, 0.99 \mathrm{~mL}$ ) was added to a solution of compound $\mathbf{S 2}$ (1 equiv, $1.8 \mathrm{mmol}$, $396 \mathrm{mg}$ ) in tetrahydrofuran $(9 \mathrm{~mL})$ at $-78^{\circ} \mathrm{C}$, and the mixture was stirred at the same temperature for $10 \mathrm{~min}$. Then, propargyl bromide ( $80 \mathrm{wt} . \%$ in toluene, 3.0 equiv, $5.4 \mathrm{mmol}, 0.6 \mathrm{~mL}$ ) was added to the reaction mixture, which was allowed to slowly warm to room temperature and stirred for $2 \mathrm{~h}$. The reaction was quenched with saturated aqueous $\mathrm{NH}_{4} \mathrm{Cl}$ solution, poured into water, extracted with EtOAc twice, dried over $\mathrm{MgSO}_{4}$ and then concentrated in vacuo. The crude mixture was purified by flash chromatography on silica gel (hexane/EtOAc, 3:1, $\mathrm{R}_{\mathrm{f}}=0.3$ ) to give compound $\mathbf{S} \mathbf{4}$ $(1.25 \mathrm{mmol}, 340 \mathrm{mg}, 69 \%)$ as a light yellow oil. ${ }^{1} \mathrm{H} \mathrm{NMR}\left(\mathrm{CDCl}_{3}, 400 \mathrm{MHz}\right) \delta 4.50(\mathrm{t}, J=5.2 \mathrm{~Hz}, 1 \mathrm{H}), 3.48(\mathrm{~d}, J=5.3$ $\mathrm{Hz}, 2 \mathrm{H}), 3.38(\mathrm{~d}, J=4.0 \mathrm{~Hz}, 6 \mathrm{H}), 2.63(\mathrm{dd}, J=4.7,2.7 \mathrm{~Hz}, 2 \mathrm{H}), 2.01(\mathrm{t}, J=2.7 \mathrm{~Hz}, 1 \mathrm{H}), 1.47(\mathrm{~s}, 12 \mathrm{H}) ;{ }^{13} \mathrm{C} \mathrm{NMR}$ $\left(\mathrm{CDCl}_{3}, 100 \mathrm{MHz}\right) \delta 171.6,103.0,82.0,79.5,79.4,71.2,64.9,54.1$ (2C), 53.8, 28.1 (3C), 21.7; IR (neat) $v_{\max }=2935$, 1723, 1601, 1454, 1367, 1260, 1154, 1100, $1047 \mathrm{~cm}^{-1}$; HRMS (CI): calcd. for $\mathrm{C}_{14} \mathrm{H}_{25} \mathrm{O}_{5}\left([\mathrm{M}+\mathrm{H}]^{+}\right) 273.1702$, found 273.1701.

tert-Butyl 2-(2,2-dimethoxyethoxy)-5-(3-methoxyphenyl)-2-methylpent-4-ynoate (S5). 1-iodo-3-methoxybenzene (1.3 equiv, $1.3 \mathrm{mmol}, 0.17 \mathrm{~mL}$ ), bis(triphenylphosphine)palladium(II) dichloride (0.02 equiv, $0.02 \mathrm{mmol}, 14 \mathrm{mg}$ ), and copper(I) iodide (0.02 equiv, $0.02 \mathrm{mmol}, 4 \mathrm{mg}$ ) were added to a solution of compound $\mathbf{S 4}$ (1 equiv, $1.0 \mathrm{mmol}, 272 \mathrm{mg}$ ) in triethylamine $(3 \mathrm{~mL})$ at room temperature. The reaction mixture was warmed to $50{ }^{\circ} \mathrm{C}$ and stirred for $2 \mathrm{~h}$. The reaction mixture was cooled to room temperature and then filtered through a pad of Celite and rinsed with EtOAc. The combined organic phase was concentrated in vacuo. The crude mixture was purified by flash chromatography on silica gel (hexane/EtOAc, 3:1, $\left.\mathrm{R}_{\mathrm{f}}=0.40\right)$ to give compound $\mathbf{S 5}(268 \mathrm{mg}, 71 \%)$ as a light yellow oil. ${ }^{1} \mathrm{H} \mathrm{NMR}\left(\mathrm{CDCl}_{3}, 400 \mathrm{MHz}\right)$ $\delta 7.21-7.14(\mathrm{~m}, 1 \mathrm{H}), 6.97(\mathrm{dt}, J=7.6,1.2 \mathrm{~Hz}, 1 \mathrm{H}), 6.91(\mathrm{dd}, J=2.7,1.4 \mathrm{~Hz}, 1 \mathrm{H}), 6.83(\mathrm{ddd}, J=8.3,2.7,1.0 \mathrm{~Hz}, 1 \mathrm{H})$, 
$4.54(\mathrm{t}, J=5.2 \mathrm{~Hz}, 1 \mathrm{H}), 3.78(\mathrm{~s}, 3 \mathrm{H}), 3.54(\mathrm{~d}, J=5.3 \mathrm{~Hz}, 2 \mathrm{H}), 3.41(\mathrm{~s}, 3 \mathrm{H}), 3.39(\mathrm{~s}, 3 \mathrm{H}), 2.78-2.93$ (m, 2H), 1.53 (s, $3 \mathrm{H}), 1.49$ (s, 9H); ${ }^{13} \mathrm{C} \mathrm{NMR}\left(\mathrm{CDCl}_{3}, 100 \mathrm{MHz}\right) \delta 171.7,159.4,129.4,124.7,124.2,116.6,114.5,103.0,85.0,83.1$, 81.9, 80.0, 65.0, 55.3, 54.2, 53.8, 29.2, 28.1 (3C), 21.8; IR (neat) $v_{\max }=2935,1731,1574,13691288,1165,1114,1047$ $\mathrm{cm}^{-1}$; HRMS (CI): calcd. for $\mathrm{C}_{21} \mathrm{H}_{29} \mathrm{O}_{6}\left([\mathrm{M}-\mathrm{H}]^{+}\right)$377.1964, found 377.1965.

tert-Butyl 2-(2,2-dimethoxyethoxy)-5-(3-methoxyphenyl)-2-methylpentanoate (6n). $10 \mathrm{wt} \% \mathrm{Pd} / \mathrm{C}$ was added to a solution of compound $\mathbf{S 5}$ (1 equiv, $0.53 \mathrm{mmol}, 200 \mathrm{mg}$ ) in ethanol $(4 \mathrm{~mL}$ ) at room temperature. The reaction mixture was bubbled with $\mathrm{H}_{2}$ gas for $3 \mathrm{~h}$, and then filtered through a pad of Celite and rinsed with EtOAc. The combined organic phase was concentrated under reduced pressure. The crude mixture was purified by flash chromatography on silica gel (hexane/EtOAc, 4:1, $\left.\mathrm{R}_{\mathrm{f}}=0.40\right)$ to give compound $\mathbf{6 n}(192 \mathrm{mg}, 95 \%)$ as a colorless oil. ${ }^{1} \mathrm{H} \mathrm{NMR}\left(\mathrm{CDCl}_{3}, 400 \mathrm{MHz}\right) \delta$ 7.14-7.22 (m, 1H), 6.68-6.78 (m, 3H), 4.49 (t, $J=5.2 \mathrm{~Hz}, 1 \mathrm{H}), 3.78$ (s, 3H), 3.43 (d, $J=5.1 \mathrm{~Hz}, 2 \mathrm{H}), 3.40(\mathrm{~s}, 3 \mathrm{H}), 3.39$ (s, 3H), $2.58(\mathrm{td}, J=6.4,2.5 \mathrm{~Hz}, 2 \mathrm{H}), 1.58-1.77(\mathrm{~m}, 4 \mathrm{H}), 1.44(\mathrm{~s}, 9 \mathrm{H}), 1.33(\mathrm{~s}, 3 \mathrm{H}) ;{ }^{13} \mathrm{C} \mathrm{NMR}\left(\mathrm{CDCl}_{3}, 100 \mathrm{MHz}\right) \delta$ 173.3, 159.7, 143.9, 129.3, 120.9, 114.3, 111.1, 103.4, 81.2, 80.4, 64.5, 55.2, 54.3, 54.0, 38.2, 36.1, 28.1 (3C), 25.2, 21.2; IR (neat) $v_{\max }=2934,1726,1602,1585,1488,1368,1258,1152,1151,1110 \mathrm{~cm}^{-1} ; \mathrm{HRMS}(\mathrm{CI}):$ calcd. for $\mathrm{C}_{21} \mathrm{H}_{33} \mathrm{O}_{6}$ $\left([\mathrm{M}-\mathrm{H}]^{+}\right)$381.2277, found 381.2277.
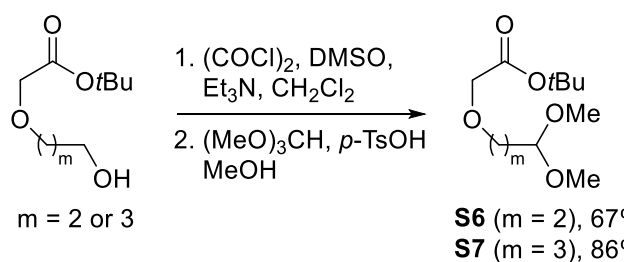

S6 $(m=2), 67 \%$ S7 $(m=3), 86 \%$

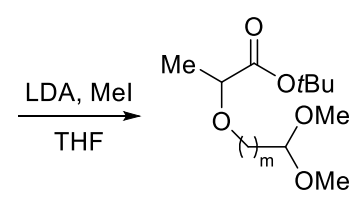

S8 $(m=2), 86 \%$ S9 $(m=3), 71 \%$

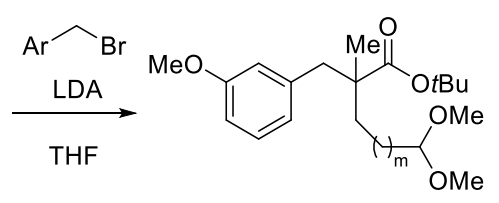

$60(m=2), 49 \%$ $6 p(m=3), 72 \%$

\section{Scheme S4. Synthesis of Friedel-Crafts substrates 60 and $\mathbf{6 p}$}

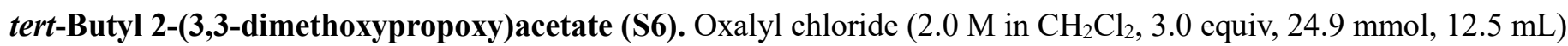
was added to a solution of DMSO (4.0 equiv, $33.2 \mathrm{mmol}, 2.4 \mathrm{~mL})$ in dichloromethane $(9 \mathrm{~mL})$ at $-78^{\circ} \mathrm{C}$, and the mixture was stirred at the same temperature for $30 \mathrm{~min}$. A solution of tert-butyl 2-(3-hydroxypropoxy)acetate ${ }^{10}$ (1.0 equiv, 8.3 mmol, $1.58 \mathrm{~g})$ in dichloromethane $(20 \mathrm{~mL})$ was added to the reaction mixture at $-78{ }^{\circ} \mathrm{C}$, and the mixture was stirred at the same temperature for $30 \mathrm{~min}$. Then, triethylamine ( 8.0 equiv, $66.4 \mathrm{mmol}, 9.2 \mathrm{~mL}$ ) was added to the reaction mixture at $-78{ }^{\circ} \mathrm{C}$, which was allowed to slowly warm to room temperature and stirred for $2 \mathrm{~h}$. The reaction was quenched with saturated aqueous $\mathrm{NH}_{4} \mathrm{Cl}$ solution, poured into water, extracted with EtOAc twice, dried over $\mathrm{MgSO}_{4}$ and then concentrated in vacuo. The residue was filtered through a pad of silica gel and rinsed with EtOAc. The crude mixture was used in next step without further purification. Trimethoxymethane (5.0 equiv, $41.5 \mathrm{mmol}, 4.5 \mathrm{~mL})$ and $p$ toluenesulfonic acid ( 0.05 equiv, $0.42 \mathrm{mmol}, 79 \mathrm{mg}$ ) were added to a solution of crude mixture in methanol $(40 \mathrm{~mL})$ at room temperature. The reaction mixture was stirred for $10 \mathrm{~min}$, quenched with saturated aqueous $\mathrm{NaHCO}_{3}$ solution, poured into water, extracted with EtOAc twice, dried over $\mathrm{MgSO}_{4}$ and then concentrated in vacuo. The crude mixture was purified by flash chromatography on silica gel (hexane/EtOAc, 3:1, $\left.\mathrm{R}_{\mathrm{f}}=0.40\right)$ to give compound $\mathbf{S 6}(1.30 \mathrm{~g}, 67 \%)$ as a colorless oil. ${ }^{1} \mathrm{H} \mathrm{NMR}\left(\mathrm{CDCl}_{3}, 400 \mathrm{MHz}\right) \delta 4.55(\mathrm{t}, J=5.8 \mathrm{~Hz}, 1 \mathrm{H}), 3.93(\mathrm{~s}, 2 \mathrm{H}), 3.57(\mathrm{t}, J=6.5 \mathrm{~Hz}, 2 \mathrm{H}), 3.34$ (s, $6 \mathrm{H}), 1.92(\mathrm{q}, J=6.1 \mathrm{~Hz}, 2 \mathrm{H}), 1.47(\mathrm{~s}, 9 \mathrm{H}) ;{ }^{13} \mathrm{C} \mathrm{NMR}\left(\mathrm{CDCl}_{3}, 100 \mathrm{MHz}\right) \delta 169.8,102.2,81.7,76.8,69.0,67.7,53.3$,

\footnotetext{
${ }^{10}$ For the synthetic procedure of tert-butyl 2-(3-hydroxypropoxy)acetate, see: ACS Med. Chem. Lett. 2020, 11, 1539-1547. ${ }^{1} \mathrm{H}$ NMR $\left(\mathrm{CDCl}_{3}\right.$, $400 \mathrm{MHz}) 3.94$ (s, 1H), 3.79 (t, $J=5.6 \mathrm{~Hz}, 2 \mathrm{H}), 3.66$ (t, $J=5.7 \mathrm{~Hz}, 2 \mathrm{H}), 2.29$ (brs, 1H), 1.81 (p, $J=5.7 \mathrm{~Hz}, 2 \mathrm{H}), 1.46$ (s, 9H).
} 
33.2, 28.2; IR (neat) $v_{\max }=2945,1748,1368,1226,1123 \mathrm{~cm}^{-1}$; HRMS (CI): calcd. for $\mathrm{C}_{11} \mathrm{H}_{23} \mathrm{O}_{5}\left([\mathrm{M}+\mathrm{H}]^{+}\right) 235.1545$, found 235.1538 .

tert-Butyl 2-(4,4-dimethoxybutoxy)acetate (S7). According to the procedure of S6, compound S7 was synthesized using tert-butyl 2-(4-hydroxybutoxy)acetate ${ }^{11}$ (1.0 equiv, $4.9 \mathrm{mmol}, 1.00 \mathrm{~g}$ ). The crude mixture was purified by flash chromatography on silica gel (hexane/EtOAc, $\left.3: 1, \mathbf{R}_{\mathrm{f}}=0.40\right)$ to give compound $\mathbf{S} 7(1.05 \mathrm{~g}, 86 \%)$ as a colorless oil. ${ }^{1} \mathrm{H}$ NMR $\left(\mathrm{CDCl}_{3}, 400 \mathrm{MHz}\right) \delta 4.36-4.43(\mathrm{~m}, 1 \mathrm{H}), 3.94(\mathrm{~s}, 2 \mathrm{H}), 3.50-3.57(\mathrm{~m}, 2 \mathrm{H}), 3.32(\mathrm{~s}, 6 \mathrm{H}), 1.69(\mathrm{t}, J=3.0 \mathrm{~Hz}, 4 \mathrm{H})$, 1.47 (s, 9H); ${ }^{13} \mathrm{C} \mathrm{NMR}\left(\mathrm{CDCl}_{3}, 100 \mathrm{MHz}\right) \delta 169.85,104.45,81.54,71.33,68.82,52.84$ (2C), 29.21, 28.18(3C), 24.84; IR (neat) $v_{\max }=2940,1747,1369,1228,1106 \mathrm{~cm}^{-1}$; HRMS (CI): calcd. for $\mathrm{C}_{12} \mathrm{H}_{23} \mathrm{O}_{5}\left([\mathrm{M}-\mathrm{H}]^{+}\right) 247.1545$, found 247.1543.

tert-Butyl 2-(3,3-dimethoxypropoxy)propanoate (S8). According to the procedure of S2, compound S8 was synthesized using $\mathbf{S 6}$ (1.0 equiv, $1.8 \mathrm{mmol}, 425 \mathrm{mg}$ ). The crude mixture was purified by flash chromatography on silica gel (hexane/EtOAc, $\left.4: 1, \mathrm{R}_{\mathrm{f}}=0.30\right)$ to give compound $\mathbf{S 8}(387 \mathrm{mg}, 86 \%)$ as a colorless oil. $\delta 4.55(\mathrm{t}, J=5.8 \mathrm{~Hz}, 1 \mathrm{H})$, 3.80 (q, $J=6.8 \mathrm{~Hz}, 1 \mathrm{H}), 3.62(\mathrm{dt}, J=9.1,6.3 \mathrm{~Hz}, 1 \mathrm{H}), 3.42$ (dt, $J=9.1,6.5 \mathrm{~Hz}, 1 \mathrm{H}), 3.34(\mathrm{~s}, 3 \mathrm{H}), 3.33$ (s, 3H), $1.84-$

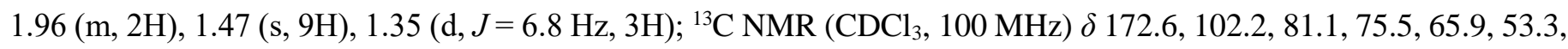
52.9, 33.2, 28.0 (3C), 18.6; IR (neat) $v_{\max }=2979,1741,1453,1368,1219,1141,1071 \mathrm{~cm}^{-1}$; HRMS (CI): calcd. for $\mathrm{C}_{12} \mathrm{H}_{25} \mathrm{O}_{5}\left([\mathrm{M}+\mathrm{H}]^{+}\right) 249.1702$, found 249.1695.

tert-Butyl 2-(4,4-dimethoxybutoxy)propanoate (S9). According to the procedure of S2, compound S9 was synthesized using S7 (1.0 equiv, $4.2 \mathrm{mmol}, 1.04 \mathrm{~g}$ ). The crude mixture was purified by flash chromatography on silica gel (hexane/EtOAc, $\left.4: 1, \mathrm{R}_{\mathrm{f}}=0.30\right)$ to give compound $\mathbf{S 9}(780 \mathrm{mg}, 71 \%)$ as a colorless oil. ${ }^{1} \mathrm{H}$ NMR $\left(\mathrm{CDCl}_{3}, 400 \mathrm{MHz}\right) \delta$ 4.38 (t, $J=5.4 \mathrm{~Hz}, 1 \mathrm{H}), 3.79$ (q, $J=6.9 \mathrm{~Hz}, 1 \mathrm{H}), 3.56$ (dt, $J=8.9,6.0 \mathrm{~Hz}, 1 \mathrm{H}), 3.35$ (dt, $J=9.2,6.1 \mathrm{~Hz}, 1 \mathrm{H}), 3.31$ (s, $6 \mathrm{H}), 1.65(\mathrm{~m}, 4 \mathrm{H}), 1.46(\mathrm{~s}, 9 \mathrm{H}), 1.34(\mathrm{~d}, J=6.7 \mathrm{~Hz}, 3 \mathrm{H}) ;{ }^{13} \mathrm{C} \mathrm{NMR}\left(\mathrm{CDCl}_{3}, 100 \mathrm{MHz}\right) \delta 172.9,104.5,81.2,75.5,69.7$, 52.9, 52.8, 29.3, 28.2 (3C), 25.1, 18.8; IR (neat) $v_{\max }=2980,1728,1368,1118,1056 \mathrm{~cm}^{-1}$; HRMS (CI): calcd. for $\mathrm{C}_{13} \mathrm{H}_{25} \mathrm{O}_{5}\left([\mathrm{M}-\mathrm{H}]^{+}\right)$261.1702, found 261.1699.

tert-Butyl 2-(3,3-dimethoxypropoxy)-3-(3-methoxyphenyl)-2-methylpropanoate (6o). According to the procedure of $\mathbf{1}$, compound $\mathbf{6 0}$ was synthesized using $\mathbf{S 8}$ (1.0 equiv, $0.8 \mathrm{mmol}, 200 \mathrm{mg}$ ). The crude mixture was purified by flash chromatography on silica gel (hexane/EtOAc, $4: 1, \mathrm{R}_{\mathrm{f}}=0.30$ ) to give compound $\mathbf{6 o}\left(145 \mathrm{mg}, 49 \%\right.$ ) as a colorless oil. ${ }^{1} \mathrm{H}$ $\operatorname{NMR}\left(\mathrm{CDCl}_{3}, 400 \mathrm{MHz}\right) \delta 7.16(\mathrm{t}, J=8.0 \mathrm{~Hz}, 1 \mathrm{H}), 6.81(\mathrm{dt}, J=3.7,1.6 \mathrm{~Hz}, 2 \mathrm{H}), 6.72-6.80(\mathrm{~m}, 1 \mathrm{H}), 4.54(\mathrm{t}, J=5.7$ $\mathrm{Hz}, 1 \mathrm{H}), 3.78$ (s, 3H), 3.47 (t, $J=6.2 \mathrm{~Hz}, 2 \mathrm{H}), 3.33(\mathrm{~s}, 3 \mathrm{H}), 3.30(\mathrm{~s}, 3 \mathrm{H}), 3.02-2.89$ (m, 2H), 1.81-1.95 (m, 2H), 1.43 (s, 9H), $1.28(\mathrm{~s}, 3 \mathrm{H}) ;{ }^{13} \mathrm{C} \mathrm{NMR}\left(\mathrm{CDCl}_{3}, 100 \mathrm{MHz}\right) \delta 173.2,159.2,138.2,128.8,123.3,116.6,112.0,102.4,81.4,80.6$, $60.3,55.2,53.3,53.2,45.2,33.7,28.1$ (3C), 20.5; IR (neat) $v_{\max }=2935,1723,1601,1584,1489,1454,1260,1153$, 1101, $1047 \mathrm{~cm}^{-1}$; HRMS (FAB): calcd. for $\mathrm{C}_{20} \mathrm{H}_{32} \mathrm{O}_{6}\left([\mathrm{M}]^{+}\right) 368.2199$, found 368.2193.

tert-Butyl 2-(4,4-dimethoxybutoxy)-3-(3-methoxyphenyl)-2-methylpropanoate (6p). According to the procedure of 1, compound $\mathbf{6 p}$ was synthesized using $\mathbf{S 9}$ (1.0 equiv, $0.8 \mathrm{mmol}, 200 \mathrm{mg}$ ). The crude mixture was purified by flash chromatography on silica gel (hexane/EtOAc, $4: 1, \mathrm{R}_{\mathrm{f}}=0.30$ ) to give compound $\mathbf{6 p}(240 \mathrm{mg}, 72 \%)$ as a colorless oil. ${ }^{1} \mathrm{H}$ $\operatorname{NMR}\left(\mathrm{CDCl}_{3}, 400 \mathrm{MHz}\right) \delta 7.16(\mathrm{t}, J=8.0 \mathrm{~Hz}, 1 \mathrm{H}), 6.72-6.84(\mathrm{~m}, 3 \mathrm{H}), 4.39(\mathrm{t}, J=5.5 \mathrm{~Hz}, 1 \mathrm{H}), 3.78(\mathrm{~s}, 3 \mathrm{H}), 3.41(\mathrm{td}$,

${ }^{11}$ For the synthetic procedure of tert-butyl 2-(4-hydroxybutoxy)acetate, see: $J$. Med. Chem. 2018, 61, 583-598. ${ }^{1} \mathrm{H} \mathrm{NMR}\left(\mathrm{CDCl}_{3}, 400 \mathrm{MHz}\right)$ $3.96(\mathrm{~s}, 2 \mathrm{H}), 3.67$ (t, $J=5.8 \mathrm{~Hz}, 2 \mathrm{H}), 3.56$ (t, $J=5.7 \mathrm{~Hz}, 2 \mathrm{H}), 1.97$ (brs, 1H), 1.77-1.60 (m, 4H), 1.47 (s, 9H). 
$J=6.0,1.4 \mathrm{~Hz}, 2 \mathrm{H}), 3.31(\mathrm{~s}, 6 \mathrm{H}), 2.90-3.03(\mathrm{~m}, 2 \mathrm{H}), 1.60-1.76(\mathrm{~m}, 4 \mathrm{H}), 1.43(\mathrm{~s}, 9 \mathrm{H}), 1.28(\mathrm{~s}, 3 \mathrm{H}) ;{ }^{13} \mathrm{C} \mathrm{NMR}\left(\mathrm{CDCl}_{3}\right.$, $100 \mathrm{MHz}) \delta 173.3,159.2,138.3,128.8,123.3,116.5,112.1,104.6,81.3,80.6,64.0,55.2,52.8$ (2C), 45.2, 29.5, 28.1 (3C), 25.6, 20.6; IR (neat) $v_{\max }=2933,1726,1602,1490,1262,1157,1113 \mathrm{~cm}^{-1}$; HRMS (FAB): calcd. for $\mathrm{C}_{21} \mathrm{H}_{34} \mathrm{O}_{6}$ $\left([\mathrm{M}]^{+}\right)$382.2355, found 382.2346.

\section{Synthesis of oxygen-bridged bicyclic products}

General procedure for the optimization of reaction conditions (Table 1): The Lewis or Brønsted acid was added to a solution of compound 1 (1 equiv, $0.1 \mathrm{mmol}, 35 \mathrm{mg}$ ) in dichloromethane $(2 \mathrm{~mL}, 0.05 \mathrm{M})$. The reaction mixture was stirred under the optimized condition given in Table 1, quenched with saturated aqueous $\mathrm{NaHCO}_{3}$ solution, poured into water, extracted with EtOAc twice, dried over $\mathrm{MgSO}_{4}$ and then concentrated in vacuo. The chemical yield and regioisomeric ratio (rr) were estimated by ${ }^{1} \mathrm{H}$ NMR analysis of crude reaction mixtures using dibromomethane $\left(\mathrm{CH}_{2} \mathrm{Br}_{2}\right)$ as an internal standard.

Scale-up reaction for the synthesis of compound 2: Triflimidic acid (2 equiv, $2.49 \mathrm{mmol}, 701 \mathrm{mg}$ ) was added to a solution of compound 1 (1 equiv, $1.25 \mathrm{mmol}, 422 \mathrm{mg}$ ) in dichloromethane $(25 \mathrm{~mL}, 0.05 \mathrm{M})$ at room temperature. The reaction mixture was stirred for $0.5 \mathrm{~h}$, quenched with saturated aqueous $\mathrm{NaHCO}_{3}$ solution, poured into water, extracted with EtOAc twice, dried over $\mathrm{MgSO}_{4}$ and then concentrated in vacuo. The crude mixture was purified by flash chromatography on silica gel (hexane/EtOAc, 6:1) to give two regioisomers $\mathbf{2 a}(254 \mathrm{mg}, 87 \%)$ and $\mathbf{2 b}$ (21 $\mathrm{mg}, 7 \%$ ) as a white solid.

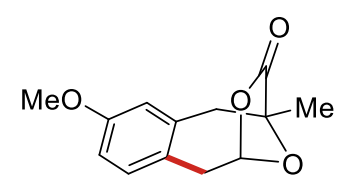

$2 \mathbf{a}$

8-Methoxy-5-methyl-1,2,5,6-tetrahydro-4H-2,5-epoxybenzo[d] oxocin-4-one (2a). $\mathrm{R}_{\mathrm{f}}=0.3$ (hexane/EtOAc, 6:1); mp $107{ }^{\circ} \mathrm{C} ;{ }^{1} \mathrm{H}$ NMR $\left(\mathrm{CDCl}_{3}, 500 \mathrm{MHz}\right) \delta 6.97(\mathrm{~d}, J=8.2 \mathrm{~Hz}, 1 \mathrm{H}), 6.69$ $(\mathrm{dd}, J=8.2,2.7 \mathrm{~Hz}, 1 \mathrm{H}), 6.60(\mathrm{~d}, J=2.7 \mathrm{~Hz}, 1 \mathrm{H}), 5.96(\mathrm{~d}, J=4.1 \mathrm{~Hz}, 1 \mathrm{H}), 3.73(\mathrm{~s}, 3 \mathrm{H}), 3.36-$ $3.41(\mathrm{~m}, 2 \mathrm{H}), 3.19(\mathrm{AB} \mathrm{q}, J=16.3 \mathrm{~Hz}, 1 \mathrm{H}) 3.18(\mathrm{dd}, J=16.2,3.9 \mathrm{~Hz}, 1 \mathrm{H}), 1.59(\mathrm{~s}, 3 \mathrm{H}) ;{ }^{13} \mathrm{C}$ $\operatorname{NMR}\left(\mathrm{CDCl}_{3}, 125 \mathrm{MHz}\right) \delta 174.7,158.7,136.3,133.4,124.7,116.7,113.0,100.3,77.4,55.4$, 47.5, 42.7, 23.3; IR (neat) $v_{\max }=2940,1785,1610,1574,1503,1250,1150 \mathrm{~cm}^{-1}$; HRMS (FAB): calcd. for $\mathrm{C}_{13} \mathrm{H}_{14} \mathrm{O}_{4}$ $\left([\mathrm{M}]^{+}\right)$234.0892, found 234.0899 .

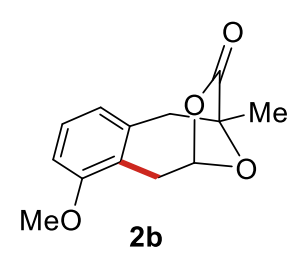

10-Methoxy-5-methyl-1,2,5,6-tetrahydro-4H-2,5-epoxybenzo[d]oxocin-4-one $(2 \mathrm{~b}) . \mathrm{R}_{\mathrm{f}}=0.35$ (hexane/EtOAc, 6:1); mp $102{ }^{\circ} \mathrm{C} ;{ }^{1} \mathrm{H}$ NMR $\left(\mathrm{CDCl}_{3}, 600 \mathrm{MHz}\right) \delta 7.12(\mathrm{t}, J=8.0 \mathrm{~Hz}, 1 \mathrm{H}), 6.78(\mathrm{~d}$, $J=8.3 \mathrm{~Hz}, 1 \mathrm{H}), 6.70(\mathrm{~d}, J=7.3 \mathrm{~Hz}, 1 \mathrm{H}), 6.03(\mathrm{~d}, J=4.1 \mathrm{~Hz}, 1 \mathrm{H}), 3.99$ (dd, $J=17.2,4.4 \mathrm{~Hz}, 1 \mathrm{H})$, 3.80 (s, 3H), 3.42 (d, $J=16.0 \mathrm{~Hz}, 1 \mathrm{H}), 3.09$ (d, $J=16.0 \mathrm{~Hz}, 1 \mathrm{H}), 2.88$ (d, $J=17.4 \mathrm{~Hz}, 1 \mathrm{H}), 1.61$ $(\mathrm{s}, 3 \mathrm{H}) ;{ }^{13} \mathrm{C} \mathrm{NMR}\left(\mathrm{CDCl}_{3}, 125 \mathrm{MHz}\right) \delta 174.7,158.3,136.8,128.0,123.4,121.7,109.7,100.5,76.9$, 55.9, 47.7, 33.4, 23.0; $v_{\max }=2901,1779,1581,1461,1253,1153,958 \mathrm{~cm}^{-1} ;$ HRMS (FAB): calcd. for $\mathrm{C}_{13} \mathrm{H}_{14} \mathrm{O}_{4}\left([\mathrm{M}]^{+}\right)$ 234.0892 , found 234.0899 .

General procedure for the synthesis of compound 7 (Table 2): Triflimidic acid (2 equiv, $0.4 \mathrm{mmol}, 112 \mathrm{mg}$ ) was added to a solution of compound 6 ( 1 equiv, $0.2 \mathrm{mmol}$ ) in dichloromethane $(4 \mathrm{~mL}, 0.05 \mathrm{M})$ at room temperature. The reaction mixture was stirred under the optimized conditions given in Table 2, quenched with saturated aqueous $\mathrm{NaHCO}_{3}$ solution, poured into water, extracted with EtOAc twice, dried over $\mathrm{MgSO}_{4}$ and then concentrated in vacuo. The crude mixture was purified by flash chromatography on silica gel. 


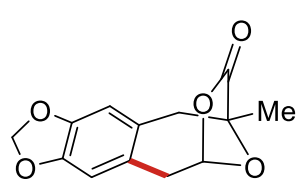

$7 a$

9-Methyl-5,6,9,10-tetrahydro-8H-6,9-epoxy[1,3]dioxolo[4',5' :4,5]benzo[1,2-d] oxocin-8-one

(7a). According to the procedure of $\mathbf{2}$, compound $7 \mathbf{a}$ was synthesized using $0.2 \mathrm{mmol}$ of $\mathbf{6 a}$. The crude mixture was purified by flash chromatography on silica gel (hexane/EtOAc, $5: 1, \mathrm{R}_{\mathrm{f}}=0.28$ ) to give 7a (46 mg, 93\%, $\mathrm{rr} 1: 0$ ) as a white solid. $\mathrm{mp} 132{ }^{\circ} \mathrm{C}$; ${ }^{1} \mathrm{H}$ NMR (major regioisomer, $\mathrm{CDCl}_{3}$, $400 \mathrm{MHz}) \delta 6.55(\mathrm{~s}, 1 \mathrm{H}), 6.53(\mathrm{~s}, 1 \mathrm{H}), 5.94(\mathrm{~d}, J=4.1 \mathrm{~Hz}, 1 \mathrm{H}), 5.91(\mathrm{~d}, J=1.4 \mathrm{~Hz}, 1 \mathrm{H}), 5.89(\mathrm{~d}$, $J=1.4 \mathrm{~Hz}, 1 \mathrm{H}), 3.34(\mathrm{~d}, J=17.2 \mathrm{~Hz}, 1 \mathrm{H}), 3.15(\mathrm{AB} \mathrm{q}, J=16.0 \mathrm{~Hz}, 2 \mathrm{H}), 3.10(\mathrm{dd}, J=16.2,4.2 \mathrm{~Hz}, 1 \mathrm{H}), 1.56(\mathrm{~d}, J=$ $7.8 \mathrm{~Hz}, 3 \mathrm{H}$ ); ${ }^{13} \mathrm{C}$ NMR (major regioisomer, $\left.\mathrm{CDCl}_{3}, 100 \mathrm{MHz}\right) \delta 174.7,147.2,146.9,128.5,126.2,112.0,111.3,101.5$, 100.0, 77.3, 47.0, 43.2, 23.0; IR (neat) $v_{\max }=2921,1785,1502,1373,1245,1132,1035 \mathrm{~cm}^{-1}$; HRMS (FAB): calcd. for $\mathrm{C}_{13} \mathrm{H}_{12} \mathrm{O}_{5}\left([\mathrm{M}]^{+}\right)$248.0685, found 248.0685.

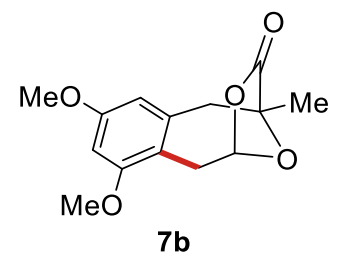

\section{8,10-Dimethoxy-5-methyl-1,2,5,6-tetrahydro-4H-2,5-epoxybenzo[d]oxocin-4-one} According to the procedure of $\mathbf{2}$, compound $\mathbf{7 b}$ was synthesized using $0.2 \mathrm{mmol}$ of $\mathbf{6 b}$. Instead, the reaction was performed under more diluted condition $(0.01 \mathrm{M})$ for $1 \mathrm{~h}$. The crude mixture was purified by flash chromatography on silica gel (hexane/EtOAc, 5:1, $\mathrm{R}_{\mathrm{f}}=0.25$ ) to give $\mathbf{7 b}$ (46 mg, 88\%) as a white solid. mp $120{ }^{\circ} \mathrm{C} ;{ }^{1} \mathrm{H}$ NMR $\left(\mathrm{CDCl}_{3}, 400 \mathrm{MHz}\right) \delta 6.34(\mathrm{~d}, J=2.3 \mathrm{~Hz}$,

$1 \mathrm{H}), 6.20(\mathrm{~d}, J=1.8 \mathrm{~Hz}, 1 \mathrm{H}), 5.98(\mathrm{~d}, J=3.7 \mathrm{~Hz}, 1 \mathrm{H}), 3.85(\mathrm{dd}, J=17.0,4.1 \mathrm{~Hz}, 1 \mathrm{H}), 3.75(\mathrm{~s}, 3 \mathrm{H}), 3.74(\mathrm{~s}, 3 \mathrm{H}), 3.21$ $(\mathrm{AB} \mathrm{q}, J=16.1 \mathrm{~Hz}, 2 \mathrm{H}), 2.79(\mathrm{~d}, J=17.0 \mathrm{~Hz}, 1 \mathrm{H}), 1.58(\mathrm{~s}, 3 \mathrm{H}) ;{ }^{13} \mathrm{C} \mathrm{NMR}\left(\mathrm{CDCl}_{3}, 100 \mathrm{MHz}\right) \delta 174.8,159.29,159.27$, 137.3, 113.9, 107.2, 100.6, 97.7, 76.9, 55.9, 55.4, 48.0, 32.9, 23.1; IR (neat) $v_{\max }=2974,1783,1582,1314,1195,1144$ $\mathrm{cm}^{-1}$; HRMS (FAB): calcd. for $\mathrm{C}_{14} \mathrm{H}_{16} \mathrm{O}_{5}\left([\mathrm{M}]^{+}\right)$264.0998, found 264.0996 .

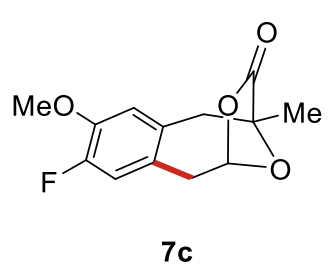

9-Fluoro-8-methoxy-5-methyl-1,2,5,6-tetrahydro-4H-2,5-epoxybenzo[d]oxocin-4-one (7c). According to the procedure of $\mathbf{2}$, compound $\mathbf{7} \mathbf{c}$ was synthesized using $0.2 \mathrm{mmol}$ of $\mathbf{6 c}$. Instead, the reaction was performed at reflux temperature for $24 \mathrm{~h}$ and the reaction concentration was $0.01 \mathrm{M}$. The crude mixture was purified by flash chromatography on silica gel (hexane/EtOAc, 6:1, $\left.\mathrm{R}_{\mathrm{f}}=0.3\right)$ to give $7 \mathrm{c}\left(26 \mathrm{mg}, 52 \%\right.$, rr 1:0) as a white solid. $\mathrm{mp} 134{ }^{\circ} \mathrm{C} ;{ }^{1} \mathrm{H} \mathrm{NMR}\left(\mathrm{CDCl}_{3}, 400\right.$ MHz) $\delta 6.81(\mathrm{~d}, J=11.8 \mathrm{~Hz}, 1 \mathrm{H}), 6.67(\mathrm{~d}, J=8.4 \mathrm{~Hz}, 1 \mathrm{H}), 5.98$ (d, $J=3.9 \mathrm{~Hz}, 1 \mathrm{H}), 3.84(\mathrm{~s}, 3 \mathrm{H}), 3.40$ (m, 2H), 3.13 $(\mathrm{dd}, J=16.4,4.0 \mathrm{~Hz}, 1 \mathrm{H}), 3.01(\mathrm{~d}, J=16.1 \mathrm{~Hz}, 1 \mathrm{H}), 1.61(\mathrm{~s}, 3 \mathrm{H}) ;{ }^{13} \mathrm{C} \mathrm{NMR}\left(\mathrm{CDCl}_{3}, 100 \mathrm{MHz}\right) \delta 174.5,152.5,150.1$, $146.18(\mathrm{~d}, J=10.6 \mathrm{~Hz}), 131.10$ (d, $J=3.8 \mathrm{~Hz}), 125.39$ (d, $J=6.3 \mathrm{~Hz}), 119.66$ (d, $J=18.7 \mathrm{~Hz}), 116.38$ (d, $J=2.4 \mathrm{~Hz})$, 100.0, 56.4, 47.0, 42.5, 23.1; IR (neat) $v_{\max }=2949,1793,1525,1213,980 \mathrm{~cm}^{-1} ; \mathrm{HRMS}$ (EI): calcd. for $\mathrm{C}_{13} \mathrm{H}_{13} \mathrm{FO}_{4}\left([\mathrm{M}]^{+}\right)$ 252.0781, found 252.0776.

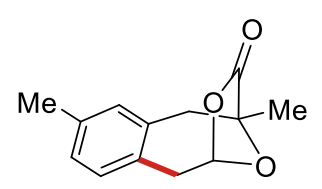

7d

5,8-Dimethyl-1,2,5,6-tetrahydro-4H-2,5-epoxybenzo[d]oxocin-4-one (7d). According to the procedure of $\mathbf{2}$, compound $\mathbf{7 d}$ was synthesized using $0.2 \mathrm{mmol}$ of $\mathbf{6 d}$. Instead, the reaction was performed for $3.5 \mathrm{~h}$ using 20 equiv of methanesulfonic acid. The crude mixture was purified by flash chromatography on silica gel (hexane/EtOAc, 5:1, $\left.\mathrm{R}_{\mathrm{f}}=0.3\right)$ to give $7 \mathbf{d}(17 \mathrm{mg}, 38 \%$, rr 18:1) as a white solid. $\mathrm{mp} 110-11{ }^{\circ} \mathrm{C} ;{ }^{1} \mathrm{H}$ NMR (major regioisomer, $\left.\mathrm{CDCl}_{3}, 400 \mathrm{MHz}\right) \delta 6.94-6.98(\mathrm{~m}$, 2H), $6.89(\mathrm{~s}, 1 \mathrm{H}), 5.98(\mathrm{~d}, J=4.1 \mathrm{~Hz}, 1 \mathrm{H}), 3.40(\mathrm{dd}, J=16.0,12.8 \mathrm{~Hz}, 2 \mathrm{H}), 3.20$ (dd, $J=16.5,4.1 \mathrm{~Hz}, 1 \mathrm{H}), 3.00(\mathrm{~d}, J$ $=16.0 \mathrm{~Hz}, 1 \mathrm{H}), 2.25(\mathrm{~s}, 3 \mathrm{H}), 1.59(\mathrm{~s}, 3 \mathrm{H}) ;{ }^{13} \mathrm{C} \mathrm{NMR}$ (major regioisomer, $\left.\mathrm{CDCl}_{3}, 200 \mathrm{MHz}\right) \delta 174.8,137.1,134.8,132.2$, 132.0, 129.7, 128.5, 100.2, 77.4, 47.4, 43.1, 23.2, 21.0; IR (neat) $v_{\max }=2901,1788,1377,1252,1156,981 \mathrm{~cm}^{-1} ; \mathrm{HRMS}$ (ESI): calcd. for $\mathrm{C}_{13} \mathrm{H}_{15} \mathrm{O}_{3}\left([\mathrm{M}+\mathrm{H}]^{+}\right) 219.1016$, found 219.1026 . 


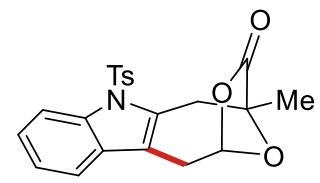

$7 h$

According to the procedure of $\mathbf{2}$, compound $\mathbf{7 h}$ was synthesized using $0.2 \mathrm{mmol}$ of $\mathbf{6 h}$. Instead, the reaction was conducted for $1 \mathrm{~h}$. The crude mixture was purified by flash chromatography on silica gel (hexane/acetone, $\left.7: 1, \mathrm{R}_{\mathrm{f}}=0.25\right)$ to give $7 \mathrm{~h}(46 \mathrm{mg}, 58 \%)$ as a white solid. $\mathrm{mp} 179{ }^{\circ} \mathrm{C}$; ${ }^{1} \mathrm{H}$ NMR $\left(\mathrm{CDCl}_{3}, 400 \mathrm{MHz}\right) \delta 8.13(\mathrm{~d}, J=8.2 \mathrm{~Hz}, 1 \mathrm{H}), 7.55(\mathrm{~d}, J=8.7 \mathrm{~Hz}, 2 \mathrm{H}), 7.27-7.32(\mathrm{~m}$, 2H), 7.17-7.22 (m, 3H), 6.15 (t, $J=2.1 \mathrm{~Hz}, 1 \mathrm{H}), 4.08$ (d, $J=18.3 \mathrm{~Hz}, 1 \mathrm{H}), 3.44$ (dd, $J=18.3,2.3 \mathrm{~Hz}, 1 \mathrm{H}), 3.31(\mathrm{~d}, J=$ $17.4 \mathrm{~Hz}, 1 \mathrm{H}), 3.14(\operatorname{app~d}, J=15.2 \mathrm{~Hz}, 1 \mathrm{H}), 2.31$ (s, 3H), 1.67 (s, 3H); ${ }^{13} \mathrm{C} \mathrm{NMR}\left(\mathrm{CDCl}_{3}, 100 \mathrm{MHz}\right) \delta 174.8,145.2$, 136.9, 135.5, 131.0, 130.5, 130.2 (2C), 126.5 (2C), 125.5, 123.9, 118.2, 115.4, 114.8, 101.2, 77.0, 39.2, 33.0, 24.4, 21.8; IR (neat) $v_{\max }=1788,1595,1454,1376,1169 \mathrm{~cm}^{-1}$; HRMS (FAB): calcd. for $\mathrm{C}_{21} \mathrm{H}_{19} \mathrm{NO}_{5} \mathrm{~S}\left([\mathrm{M}]^{+}\right) 397.0984$, found 397.0989 .

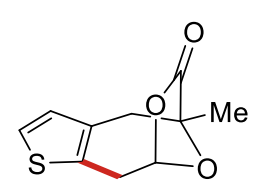

7i

5-Methyl-4,5,8,9-tetrahydro-6H-5,8-epoxythieno[2,3-d]oxocin-6-one (7i). According to the procedure of $\mathbf{2}$, compound $7 \mathbf{i}$ was synthesized using $0.2 \mathrm{mmol}$ of $\mathbf{6 i}$. Instead, the reaction was conducted at reflux temperature for $0.5 \mathrm{~h}$ and the reaction concentration was $0.01 \mathrm{M}$. The crude mixture was purified by flash chromatography on silica gel (hexane/acetone, 5:1, $\mathrm{R}_{\mathrm{f}}=0.25$ ) to give $7 \mathbf{i}$ $(36 \mathrm{mg}, 86 \%)$ as a colorless oil; ${ }^{1} \mathrm{H} \mathrm{NMR}\left(\mathrm{CDCl}_{3}, 400 \mathrm{MHz}\right) \delta 7.09(\mathrm{~d}, J=5.1 \mathrm{~Hz}, 1 \mathrm{H}), 6.69(\mathrm{~d}, J=$ $5.1 \mathrm{~Hz}, 1 \mathrm{H}), 6.07(\mathrm{t}, J=2.1 \mathrm{~Hz}, 1 \mathrm{H}), 3.14-3.46(\mathrm{~m}, 4 \mathrm{H}), 1.61(\mathrm{~s}, 3 \mathrm{H}) ;{ }^{13} \mathrm{C} \mathrm{NMR}\left(\mathrm{CDCl}_{3}, 100 \mathrm{MHz}\right) \delta 174.7,132.2$, 131.1, 130.7, 124.3, 101.5, 78.0, 39.8, 37.7, 23.4; IR (neat) $v_{\max }=2921,1793,1290,1166,983 \mathrm{~cm}^{-1}$; HRMS (FAB): calcd. for $\mathrm{C}_{10} \mathrm{H}_{11} \mathrm{O}_{3} \mathrm{~S}\left([\mathrm{M}+\mathrm{H}]^{+}\right)$211.0429, found 211.0421 .

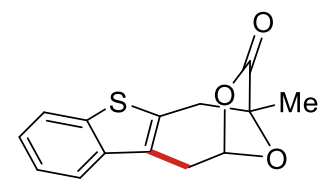

$7 \mathbf{j}$

\section{5-Methyl-1,2,5,6-tetrahydro-4H-2,5-epoxybenzo[4,5] thieno[3,2-d]oxocin-4-one}

According to the procedure of $\mathbf{2}$, compound $\mathbf{7 j}$ was synthesized using $0.2 \mathrm{mmol}$ of $\mathbf{6 j}$. Instead, 20 equiv of methanesulfonic acid was used. The crude mixture was purified by flash chromatography on silica gel (hexane/EtOAc, $\left.7: 1, \mathrm{R}_{\mathrm{f}}=0.25\right)$ to give $7 \mathbf{j}(43 \mathrm{mg}, 82 \%)$ as a white solid. $\mathrm{mp} 188{ }^{\circ} \mathrm{C}$; ${ }^{1} \mathrm{H}$ NMR $\left(\mathrm{CDCl}_{3}, 400 \mathrm{MHz}\right) \delta 7.71(\mathrm{~d}, J=8.0 \mathrm{~Hz}, 1 \mathrm{H}), 7.52(\mathrm{~d}, J=8.0 \mathrm{~Hz}, 1 \mathrm{H}), 7.28-7.37$ (m, 2H), 6.21-6.28 (m, 1H), 3.47-3.57 (m, 2H), $3.36(\mathrm{~d}, J=17.2 \mathrm{~Hz}, 1 \mathrm{H}), 3.25(\mathrm{~d}, J=17.2 \mathrm{~Hz}, 1 \mathrm{H}), 1.64(\mathrm{~s}, 3 \mathrm{H}) ;{ }^{13} \mathrm{C} \mathrm{NMR}$ $\left(\mathrm{CDCl}_{3}, 100 \mathrm{MHz}\right) \delta 174.4,141.0,139.2,133.5,124.8,124.6,124.5,122.3,121.0,101.6,77.8,40.8,35.7,23.5 ; \mathrm{IR}$ (neat) $v_{\max }=2922,1787,1433,1360,1294,1163 \mathrm{~cm}^{-1}$; HRMS (FAB): calcd. for $\mathrm{C}_{14} \mathrm{H}_{12} \mathrm{O}_{3} \mathrm{~S}\left([\mathrm{M}]^{+}\right) 260.0507$, found 260.0511 .

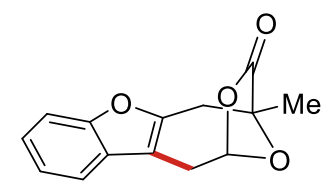

$7 \mathbf{k}$

5-Methyl-1,2,5,6-tetrahydro-4H-2,5-epoxyoxocino[5,4-b]benzofuran-4-one (7k). According to the procedure of $\mathbf{2}$, compound $\mathbf{7} \mathbf{k}$ was synthesized using $0.2 \mathrm{mmol}$ of $\mathbf{6 k}$. Instead, the reaction was conducted at reflux temperature for $0.5 \mathrm{~h}$ and the reaction concentration was $0.01 \mathrm{M}$. The crude mixture was purified by flash chromatography on silica gel (hexane/acetone, $4: 1, \mathrm{R}_{\mathrm{f}}=0.35$ ) to give $7 \mathbf{i}$ (39 mg, 81\%) as a colorless oil; ${ }^{1} \mathrm{H}$ NMR $\left(\mathrm{CDCl}_{3}, 400 \mathrm{MHz}\right) \delta 7.34-7.41(\mathrm{~m}, 2 \mathrm{H})$, 7.18-7.31 (m, 2H), $6.25(\mathrm{t}, J=2.1 \mathrm{~Hz}, 1 \mathrm{H}), 3.35-3.53(\mathrm{~m}, 2 \mathrm{H}), 3.10-3.32(\mathrm{~m}, 2 \mathrm{H}), 1.67(\mathrm{~s}, 3 \mathrm{H}) ;{ }^{13} \mathrm{C} \mathrm{NMR}\left(\mathrm{CDCl}_{3}, 100\right.$ MHz) $\delta 174.6,154.6,148.3,129.6,124.6,122.8,118.6,111.0,108.9,102.1,39.5,30.9,23.7$; IR (neat) $v_{\max }=2919$, 1797, 1457, 1174, $992 \mathrm{~cm}^{-1}$; HRMS (FAB): calcd. for $\mathrm{C}_{14} \mathrm{H}_{12} \mathrm{O}_{4}\left([\mathrm{M}]^{+}\right)$244.0736, found 244.0741. 


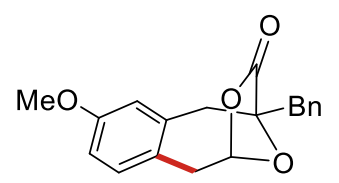

71

5-Benzyl-8-methoxy-1,2,5,6-tetrahydro-4H-2,5-epoxybenzo[d]oxocin-4-one (7l). According to the procedure of $\mathbf{2}$, compound $\mathbf{7 1}$ was synthesized using $0.2 \mathrm{mmol}$ of $\mathbf{6 1}$. The reaction was conducted for $15 \mathrm{~min}$. The crude mixture was purified by flash chromatography on silica gel (hexane/EtOAc, $\left.10: 1, \mathrm{R}_{\mathrm{f}}=0.25\right)$ to give $71\left(56 \mathrm{mg}, 91 \%\right.$, rr 12:1) as a white solid. $\mathrm{mp} 106-107^{\circ} \mathrm{C}$; ${ }^{1} \mathrm{H}$ NMR (major regioisomer, $\left.\mathrm{CDCl}_{3}, 400 \mathrm{MHz}\right) \delta 7.25-7.31(\mathrm{~m}, 5 \mathrm{H}), 6.96(\mathrm{~d}, J=8.7 \mathrm{~Hz}, 1 \mathrm{H}), 6.70(\mathrm{dd}, J=8.5,2.5$ $\mathrm{Hz}, 1 \mathrm{H}), 6.64(\mathrm{~d}, J=2.3 \mathrm{~Hz}, 1 \mathrm{H}), 5.90(\mathrm{~d}, J=3.2 \mathrm{~Hz}, 1 \mathrm{H}), 3.75(\mathrm{~s}, 3 \mathrm{H}), 3.23-3.39$ (m, 3H), 3.12-3.18 (m, 2H), $3.04(\mathrm{~d}$, $J=16.0 \mathrm{~Hz}, 1 \mathrm{H}$ ); ${ }^{13} \mathrm{C} \mathrm{NMR}$ (major regioisomer, $\left.\mathrm{CDCl}_{3}, 100 \mathrm{MHz}\right) \delta 173.9,158.7,136.2,134.8,133.4,130.8$ (2C), 128.5 (2C), 127.3, 124.6, 116.7, 113.1, 100.6, 79.9, 55.4, 46.42, 42.7, 42.3; IR (neat) $v_{\max }=1783,1504,1366,1271$, $1147 \mathrm{~cm}^{-1}$; HRMS (FAB): calcd. for $\mathrm{C}_{19} \mathrm{H}_{18} \mathrm{O}_{4}\left([\mathrm{M}]^{+}\right)$310.1205, found 310.1208 .

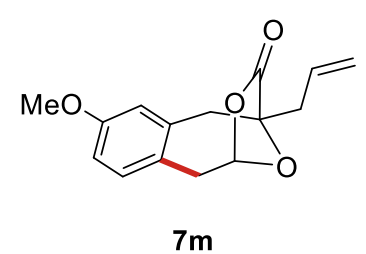

\section{5-Allyl-8-methoxy-1,2,5,6-tetrahydro-4H-2,5-epoxybenzo[d]oxocin-4-one}

(7m).

According to the procedure of $\mathbf{2}$, compound $\mathbf{7 m}$ was synthesized using $0.2 \mathrm{mmol}$ of $\mathbf{6 m}$. The crude mixture was purified by flash chromatography on silica gel (hexane/EtOAc, $8: 1, \mathrm{R}_{\mathrm{f}}=$ $0.28)$ to give $7 \mathbf{m}\left(42 \mathrm{mg}, 80 \%\right.$, rr 12:1) as a colorless oil. ${ }^{1} \mathrm{H}$ NMR (major regioisomer, $\mathrm{CDCl}_{3}$, $400 \mathrm{MHz}) \delta 6.99(\mathrm{~d}, J=8.4 \mathrm{~Hz}, 1 \mathrm{H}), 6.72(\mathrm{dd}, J=8.4,2.6 \mathrm{~Hz}, 1 \mathrm{H}), 6.64(\mathrm{~d}, J=2.5 \mathrm{~Hz}, 1 \mathrm{H}), 6.00(\mathrm{~d}, J=3.9 \mathrm{~Hz}, 1 \mathrm{H})$, $5.87(\mathrm{ddt}, J=17.3,10.1,7.2 \mathrm{~Hz}, 1 \mathrm{H}), 5.14-5.32(\mathrm{~m}, 2 \mathrm{H}), 3.76(\mathrm{~s}, 3 \mathrm{H}), 3.40(\mathrm{dd}, J=16.1,9.0 \mathrm{~Hz}, 2 \mathrm{H}), 3.20$ (dd, $J=$ 16.4, $4.0 \mathrm{~Hz}, 1 \mathrm{H}), 2.98(\mathrm{~d}, J=16.1 \mathrm{~Hz}, 1 \mathrm{H}), 2.57-2.76(\mathrm{~m}, 2 \mathrm{H}) ;{ }^{13} \mathrm{C}$ NMR (major regioisomer, $\left.\mathrm{CDCl}_{3}, 100 \mathrm{MHz}\right) \delta$ 173.8, 158.7, 136.2, 133.4, 131.1, 124.7, 120.2, 116.7, 113.1, 100.6, 79.3, 55.4, 46.1, 42.8, 40.7; IR (neat) $v_{\max }=2939$, 1788, 1609, 1504, 1247, $1144 \mathrm{~cm}^{-1}$; HRMS (FAB): calcd. for $\mathrm{C}_{15} \mathrm{H}_{16} \mathrm{O}_{4}\left([\mathrm{M}]^{+}\right)$260.1049, found 260.1049 .

\section{Synthesis of $O$-acyl hemiacetal intermediates $5 \mathbf{a}$ and $5 \mathbf{b}$}

6-Methoxy-3-(3-methoxybenzyl)-3-methyl-1,4-dioxan-2-one (5). Trifluoromethanesulfonic acid (0.2 equiv, 0.07 mmol, $6.5 \mu \mathrm{L}$ ) was added to a solution of compound $\mathbf{1}(1$ equiv, $131 \mathrm{mg}, 0.37 \mathrm{mmol})$ in dichloromethane $(7.4 \mathrm{~mL})$ using a $20 \mu \mathrm{L}$ MicroVolume syringe at room temperature. The reaction mixture was stirred at room temperature for $5 \mathrm{~min}$, quenched with saturated aqueous $\mathrm{NaHCO}_{3}$ solution, poured into water, extracted with dichloromethane twice, dried over $\mathrm{MgSO}_{4}$ and then concentrated in vacuo. The crude mixture was purified by flash chromatography on silica gel (hexane/EtOAc, 7:1) to give two diastereomers $\mathbf{5 a}(60 \mathrm{mg}, 61 \%)$ and $\mathbf{5 b}(37 \mathrm{mg}, 38 \%$ ) as a colorless oil.

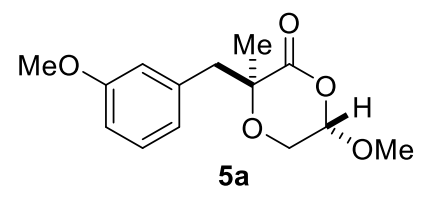

5a: $\mathrm{R}_{\mathrm{f}}=0.3$ (hexane/EtOAc, 7:1); ${ }^{1} \mathrm{H}$ NMR $\left(\mathrm{CDCl}_{3}, 600 \mathrm{MHz}\right) \delta 7.15-7.23(\mathrm{~m}, 1 \mathrm{H}), 6.83$ (dt, $J=7.8,1.4 \mathrm{~Hz}, 1 \mathrm{H}), 6.77-6.82(\mathrm{~m}, 2 \mathrm{H}), 4.78(\mathrm{dd}, J=6.9,2.8 \mathrm{~Hz}, 1 \mathrm{H}), 3.79(\mathrm{~s}, 3 \mathrm{H})$, $3.74(\mathrm{dd}, J=12.6,2.9 \mathrm{~Hz}, 1 \mathrm{H}), 3.51(\mathrm{dd}, J=12.8,6.9 \mathrm{~Hz}, 1 \mathrm{H}), 3.46(\mathrm{~s}, 3 \mathrm{H}), 3.22(\mathrm{~d}, J=$ $13.6 \mathrm{~Hz}, 1 \mathrm{H}), 2.89(\mathrm{~d}, J=13.5 \mathrm{~Hz}, 1 \mathrm{H}), 1.53(\mathrm{~s}, 3 \mathrm{H}) ;{ }^{13} \mathrm{C} \mathrm{NMR}\left(\mathrm{CDCl}_{3}, 100 \mathrm{MHz}\right) \delta 171.8$, 159.4, 137.1, 129.2, 123.1, 116.3, 112.7, 101.5, 79.4, 62.0, 57.0, 55.3, 46.0, 23.7; IR (neat) $v_{\max }=2937,1739,1583$, 1488, 1453, 1261, 1164, $1037 \mathrm{~cm}^{-1}$; HRMS (FAB): calcd. for $\mathrm{C}_{14} \mathrm{H}_{18} \mathrm{O}_{5}\left([\mathrm{M}]^{+}\right)$266.1154, found 266.1151 .

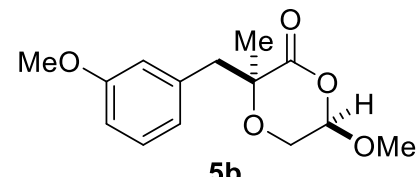

$5 b$

5b: $\mathrm{R}_{\mathrm{f}}=0.2$ (hexane/EtOAc, $\left.7: 1\right) ;{ }^{1} \mathrm{H}$ NMR $\left(\mathrm{CDCl}_{3}, 400 \mathrm{MHz}\right) \delta 7.18-7.22(\mathrm{~m}, 1 \mathrm{H}), 6.78-$ $6.50(\mathrm{~m}, 3 \mathrm{H}), 5.18(\mathrm{dd}, J=4.8,2.6 \mathrm{~Hz}, 1 \mathrm{H}), 3.85(\mathrm{dd}, J=12.7,2.6 \mathrm{~Hz}, 1 \mathrm{H}), 3.79(\mathrm{~s}, 3 \mathrm{H})$, $3.56(\mathrm{dd}, J=12.7,4.8 \mathrm{~Hz}, 1 \mathrm{H}), 3.40(\mathrm{~s}, 3 \mathrm{H}), 3.31(\mathrm{~d}, J=13.9 \mathrm{~Hz}, 1 \mathrm{H}), 2.91(\mathrm{~d}, J=13.8$ $\mathrm{Hz}, 1 \mathrm{H}), 1.45(\mathrm{~s}, 3 \mathrm{H}) ;{ }^{13} \mathrm{C} \mathrm{NMR}\left(\mathrm{CDCl}_{3}, 100 \mathrm{MHz}\right) \delta 172.0,159.4,136.9,129.1,123.4$, 
116.6, 112.5, 101.3, 79.1, 61.9, 56.7, 55.3, 43.7, 24.4; IR (neat) $v_{\max }=2936,1739,1600,1489,1452,1263,1152 \mathrm{~cm}^{-1}$; HRMS (FAB): calcd. for $\mathrm{C}_{14} \mathrm{H}_{18} \mathrm{O}_{5}\left([\mathrm{M}]^{+}\right)$266.1154, found 266.1150 . 


\section{X-ray Crystallographic Data}

\section{D-1. Crystal data for compound 2a}

Experimental details: Single crystals of compound 2a were obtained from ethyl acetate-hexane cosolvent system by slow evaporation for 1 day $\left(\mathrm{mp} 90-95^{\circ} \mathrm{C}\right)$. The single-crystal X-ray diffraction data was collected at 100.00(10)K on a SuperNova, Dual, $\mathrm{Cu}$ at home/near, AtlasS2 diffractometer. Using Olex2, ${ }^{12}$ the structure was solved with the SHELXT $^{13}$ structure solution program using Intrinsic Phasing and refined with the SHELXL ${ }^{13}$ refinement package using Least Squares minimization.

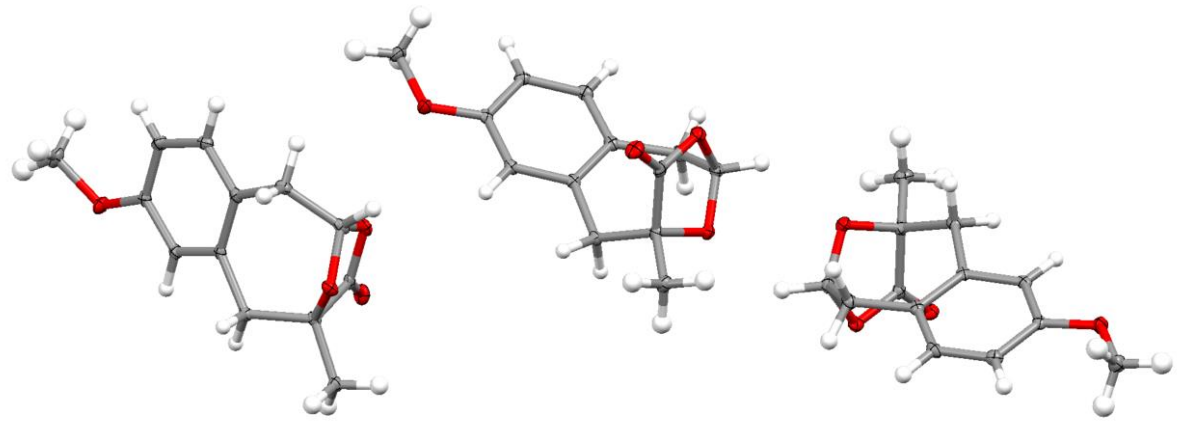

Figure S1. The molecular structure of the three independent molecules of compound $\mathbf{2 a}$; ellipsoid contour at the $30 \%$ probability level.

CCDC (1991588) contains the supplementary crystallographic data for this paper. These data can be obtained free of charge from the Cambridge Crystallographic Data Centre via www.ccdc.cam.ac.uk/data_request/cif.

Table S7. Crystal data and structure refinement for compound 2a

Identification code

Empirical formula

Formula weight

Temperature/K

Crystal system

Space group

$\mathrm{a} / \AA$

$\mathrm{b} / \AA$

$\mathrm{c} / \AA$

$\alpha /^{\circ}$

$\beta /{ }^{\circ}$

$\gamma /{ }^{\circ}$

Volume/ $\AA^{3}$

Z

$\rho_{\text {calc }} \mathrm{g} / \mathrm{cm}^{3}$

$\mu / \mathrm{mm}^{-1}$

$\mathrm{F}(000)$

$$
\begin{aligned}
& \text { exp_1427-2 } \\
& \mathrm{C}_{39} \mathrm{H}_{42} \mathrm{O}_{12} \\
& 702.72 \\
& 100.00(10) \\
& \text { monoclinic } \\
& \mathrm{P} 2_{1} / \mathrm{c} \\
& 7.7012(3) \\
& 24.7521(9) \\
& 17.4823(7) \\
& 90 \\
& 94.305(3) \\
& 90 \\
& 3323.1(2) \\
& 4 \\
& 1.405 \\
& 0.104 \\
& 1488.0
\end{aligned}
$$

12 O. V. Dolomanov, L. J. Bourhis, R. J. Gildea, J. A. K. Howard, H. Puschmann. OLEX2: a complete structure solution, refinement and analysis program. J. Appl. Cryst. 2009, 42, 339-341.

13 G. M. Sheldrick. SHELXT - Integrated space-group and crystal-structure determination. Acta Cryst. 2015, A71, 3-8. 


$\begin{array}{ll}\text { Crystal size } / \mathrm{mm}^{3} & 0.366 \times 0.268 \times 0.236 \\ \text { Radiation } & \mathrm{Mo} \mathrm{K \alpha}(\lambda=0.71073) \\ 2 \Theta \text { range for data collection } /{ }^{\circ} & 4.674 \text { to } 55 \\ \text { Index ranges } & -9 \leq \mathrm{h} \leq 10,-31 \leq \mathrm{k} \leq 32,-22 \leq 1 \leq 22 \\ \text { Reflections collected } & 40928 \\ \text { Independent reflections } & 40928\left[\mathrm{R}_{\text {int }}=?, \mathrm{R}_{\text {sigma }}=0.0586\right] \\ \text { Data/restraints/parameters } & 40928 / 0 / 467 \\ \text { Goodness-of-fit on } \mathrm{F}^{2} & 1.026 \\ \text { Final R indexes }[\mathrm{I}>=2 \sigma(\mathrm{I})] & \mathrm{R}_{1}=0.0494, \mathrm{wR}_{2}=0.1086 \\ \text { Final } \mathrm{R} \text { indexes }[\text { all data }] & \mathrm{R}_{1}=0.0658, \mathrm{wR}_{2}=0.1208 \\ \text { Largest diff. peak/hole } / \mathrm{e} \AA^{-3} & 0.27 /-0.28\end{array}$

\section{D-2. Crystal data for compound 71}

Experimental details: Single crystals of compound 7l were obtained from ethyl acetate-hexane cosolvent system by slow evaporation for 1 day $\left(\mathrm{mp} 100-105^{\circ} \mathrm{C}\right)$. The single-crystal X-ray diffraction data was collected at 100.00(10)K on a SuperNova, Dual, $\mathrm{Cu}$ at home/near, AtlasS2 diffractometer. Using Olex $2,{ }^{12}$ the structure was solved with the SHELXT ${ }^{13}$ structure solution program using Intrinsic Phasing and refined with the SHELXL ${ }^{13}$ refinement package using Least Squares minimization.

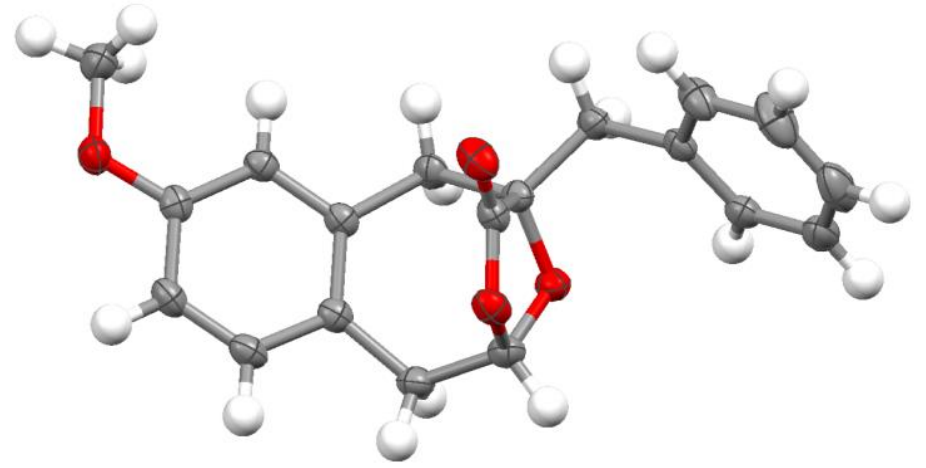

Figure S2. X-ray structure for compound 7l; ellipsoid contour at the $30 \%$ probability level.

CCDC (1991568) contains the supplementary crystallographic data for this paper. These data can be obtained free of charge from the Cambridge Crystallographic Data Centre via www.ccdc.cam.ac.uk/data_request/cif.

Table S8. Crystal data and structure refinement for compound 71

$\begin{array}{ll}\text { Identification code } & \text { exp_1424 } \\ \text { Empirical formula } & \mathrm{C}_{19} \mathrm{H}_{18} \mathrm{O}_{4} \\ \text { Formula weight } & 310.33 \\ \text { Temperature/K } & 170.00(10) \\ \text { Crystal system } & \text { monoclinic } \\ \text { Space group } & \mathrm{P} 2_{1} / \mathrm{c} \\ \mathrm{a} / \AA & 11.4432(4) \\ \mathrm{b} / \AA & 11.7458(4) \\ \mathrm{c} / \AA & 11.7368(4) \\ \alpha^{\circ} & 90\end{array}$




\begin{tabular}{|c|c|}
\hline$\beta /{ }^{\circ}$ & $101.563(4)$ \\
\hline$\gamma /{ }^{\circ}$ & 90 \\
\hline Volume $/ \AA^{3}$ & $1545.52(9)$ \\
\hline Z & 4 \\
\hline$\rho_{\text {calc }} \mathrm{g} / \mathrm{cm}^{3}$ & 1.334 \\
\hline$\mu / \mathrm{mm}^{-1}$ & 0.093 \\
\hline $\mathrm{F}(000)$ & 656.0 \\
\hline Crystal size $/ \mathrm{mm}^{3}$ & $0.562 \times 0.34 \times 0.299$ \\
\hline Radiation & Mo $\mathrm{K} \alpha(\lambda=0.71073)$ \\
\hline \multicolumn{2}{|c|}{$2 \Theta$ range for data collection $/{ }^{\circ} 4.958$ to 59.566} \\
\hline Index ranges & $-15 \leq \mathrm{h} \leq 14,-16 \leq \mathrm{k} \leq 16,-15 \leq 1 \leq 16$ \\
\hline Reflections collected & 17781 \\
\hline Independent reflections & $3926\left[\mathrm{R}_{\mathrm{int}}=0.0337, \mathrm{R}_{\text {sigma }}=0.0325\right]$ \\
\hline Data/restraints/parameters & $3926 / 0 / 209$ \\
\hline Goodness-of-fit on $\mathrm{F}^{2}$ & 1.032 \\
\hline Final $R$ indexes $[\mathrm{I}>=2 \sigma(\mathrm{I})]$ & $\mathrm{R}_{1}=0.0474, \mathrm{wR}_{2}=0.1059$ \\
\hline Final $\mathrm{R}$ indexes [all data] & $\mathrm{R}_{1}=0.0622, \mathrm{wR}_{2}=0.1150$ \\
\hline Largest diff. peak/hole / e & $0.25 /-0.29$ \\
\hline
\end{tabular}




\section{E. NMR study}

E-1. Treatment of the major diastereomer 5a with catalytic $\mathrm{SnBr}_{4}$ (Scheme 2c)

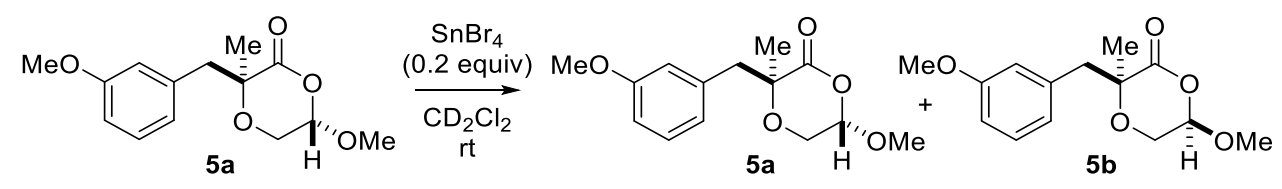

The major diastereomer $5 \mathbf{a}$ (1.0 equiv, $0.10 \mathrm{mmol}, 27 \mathrm{mg})$ in deuterated dichloromethane $\left(\mathrm{CD}_{2} \mathrm{Cl}_{2}, 2 \mathrm{~mL}\right)$ was treated with catalytic tin(IV) bromide ( 0.2 equiv, $0.020 \mathrm{mmol}, 8.8 \mathrm{mg}$ ), and the mixture was subjected to ${ }^{1} \mathrm{H}$-NMR spectroscopy at each time point (10 $\mathrm{min}, 30 \mathrm{~min}, 60 \mathrm{~min}$, and $120 \mathrm{~min})$.

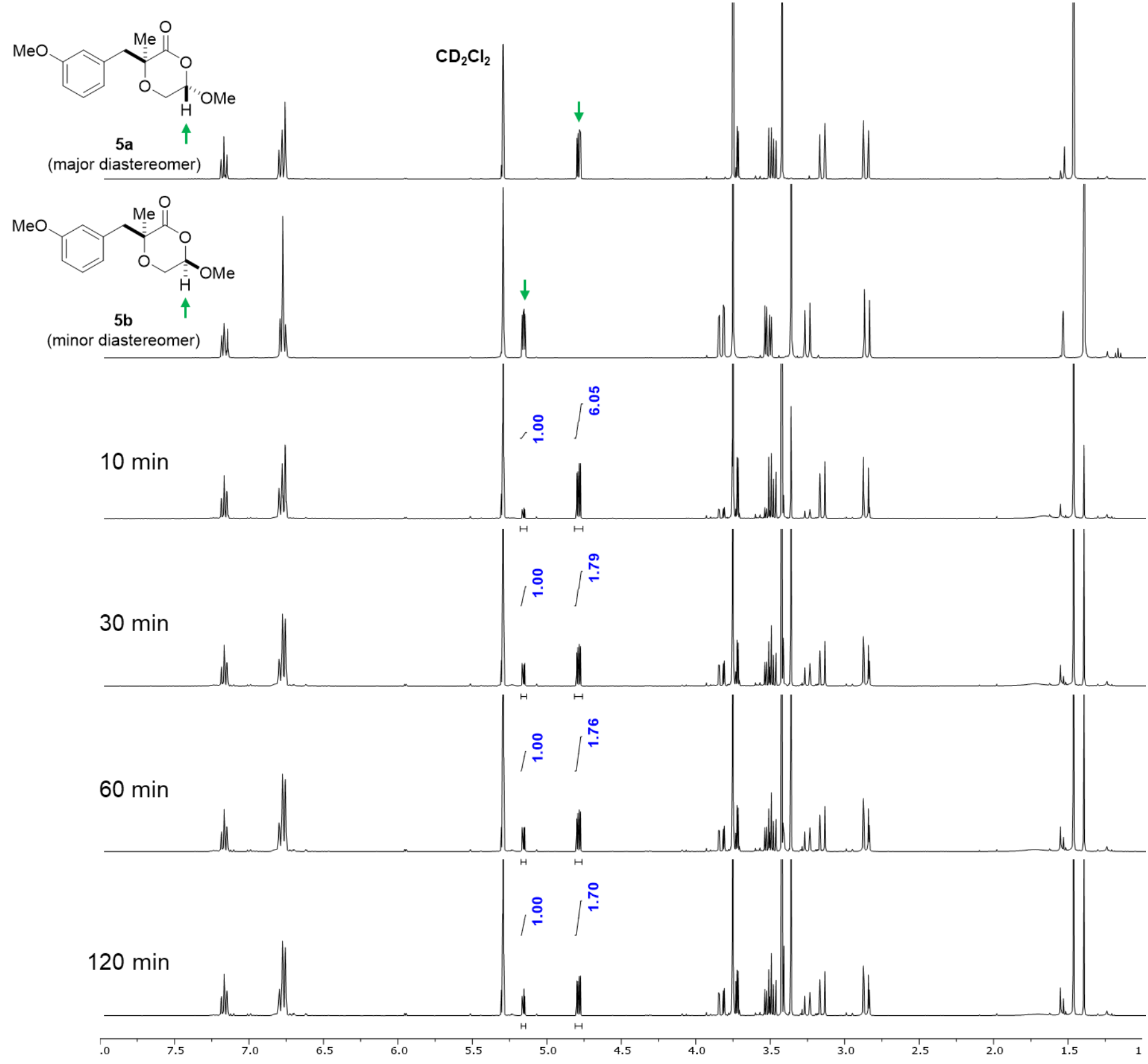

Figure S3. Time-dependent ${ }^{1} \mathrm{H}$ NMR spectra for $\mathbf{5 a}$ in the presence of catalytic $\mathrm{SnBr}_{4}$ 
E-2. Deuterium exchange experiment (Scheme 2d)

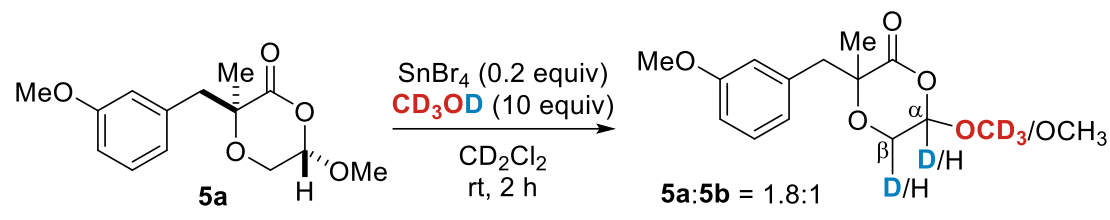

The major diastereomer $5 \mathbf{a}$ (1.0 equiv, $0.079 \mathrm{mmol}, 21 \mathrm{mg})$ in deuterated dichloromethane $\left(\mathrm{CD}_{2} \mathrm{Cl}_{2}, 1.6 \mathrm{~mL}\right)$ was treated with catalytic tin(IV) bromide in the presence of deuterated methanol $\left(\mathrm{CD}_{3} \mathrm{OD}, 10\right.$ equiv, $\left.0.79 \mathrm{mmol}, 32 \mu \mathrm{L}\right)$. After $2 \mathrm{~h}$, the organic solution was quenched by aqueous sodium bicarbonate, diluted with dichloromethane, and extracted by water to remove an unidentified water-soluble side product. The organic layer was concentrated in vacuo and directly subjected to ${ }^{1} \mathrm{H}-\mathrm{NMR}$ spectroscopy $\left(\mathrm{CD}_{2} \mathrm{Cl}_{2}\right.$ was used as the NMR solvent).
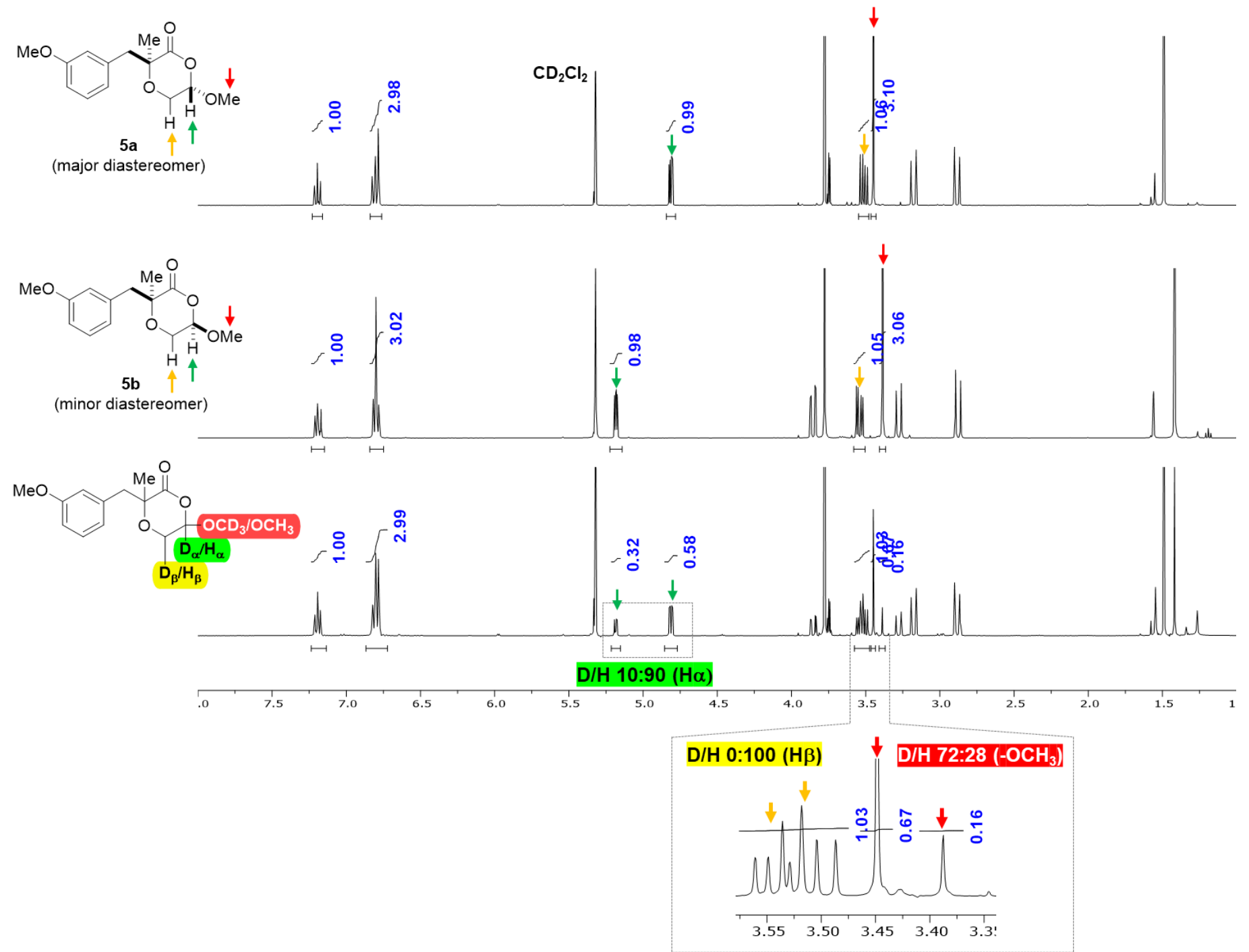

Figure S4. ${ }^{1} \mathrm{H}$ NMR spectra for the deuterium exchange experiment with $\mathbf{5 a}$ 
F. Copies of NMR data
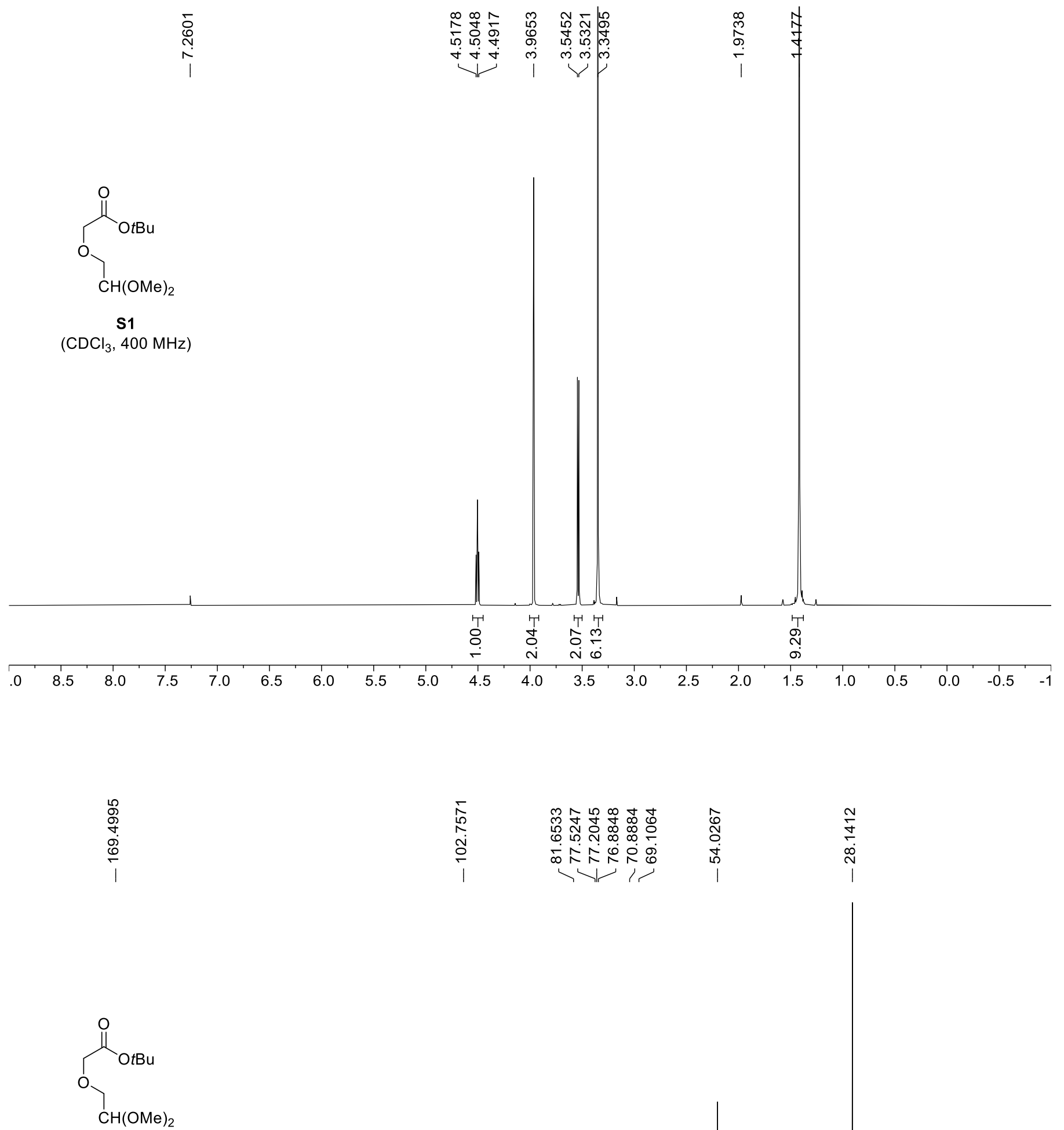

s1

$\left(\mathrm{CDCl}_{3}, 100 \mathrm{MHz}\right)$

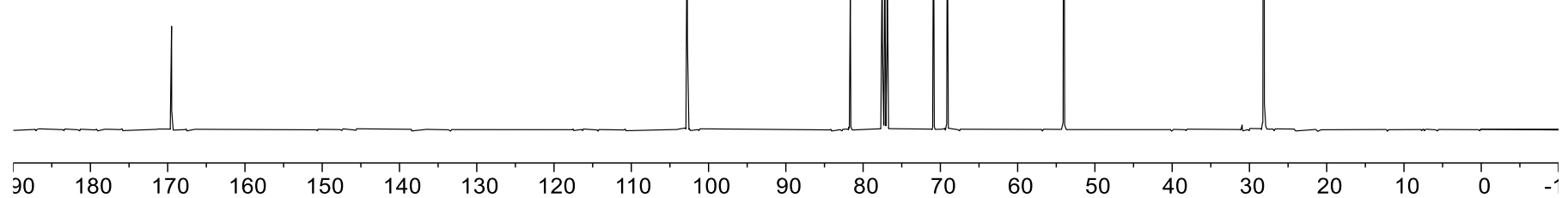


<smiles>CCCCOC(=O)C(C)OCC(C)C</smiles>

S2

(CDCl3, $400 \mathrm{MHz})$

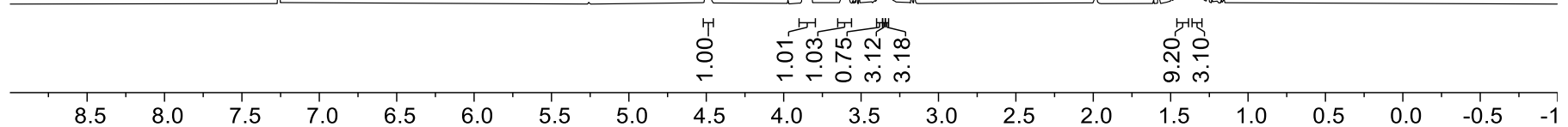<smiles>CCCCOC(=O)C(C)OCC(C)C</smiles>

S2

$\left(\mathrm{CDCl}_{3}, 100 \mathrm{MHz}\right)$

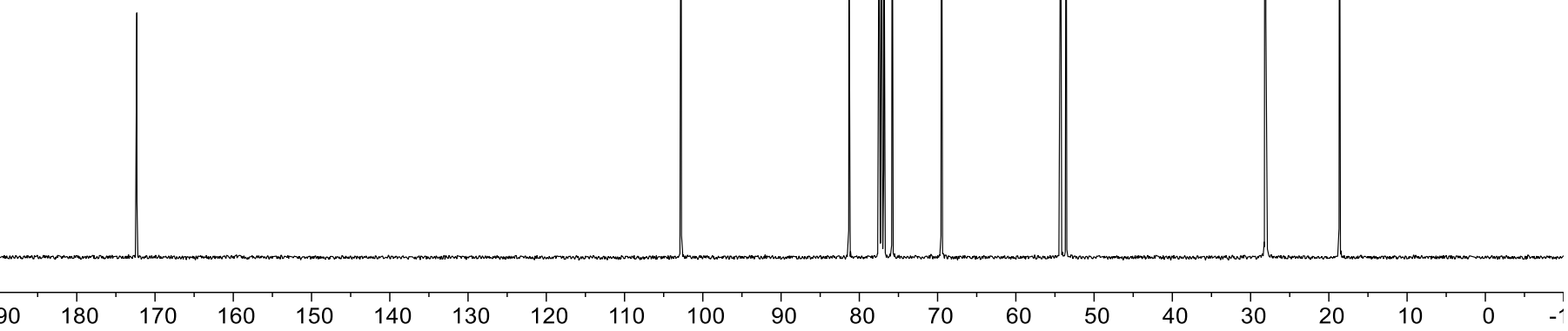


<smiles>CCOC(=O)C(C)(Cc1cccc(OC)c1)OCCOC</smiles>

$\left(\mathrm{CDCl}_{3}, 400 \mathrm{MHz}\right)$

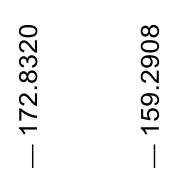

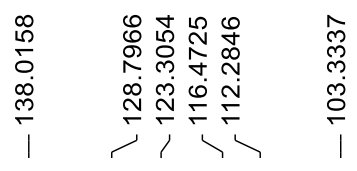<smiles>CCCOC(=O)C(C)(Cc1cccc(OC)c1)OCCC(C)C</smiles>

$\left(\mathrm{CDCl}_{3}, 100 \mathrm{MHz}\right)$

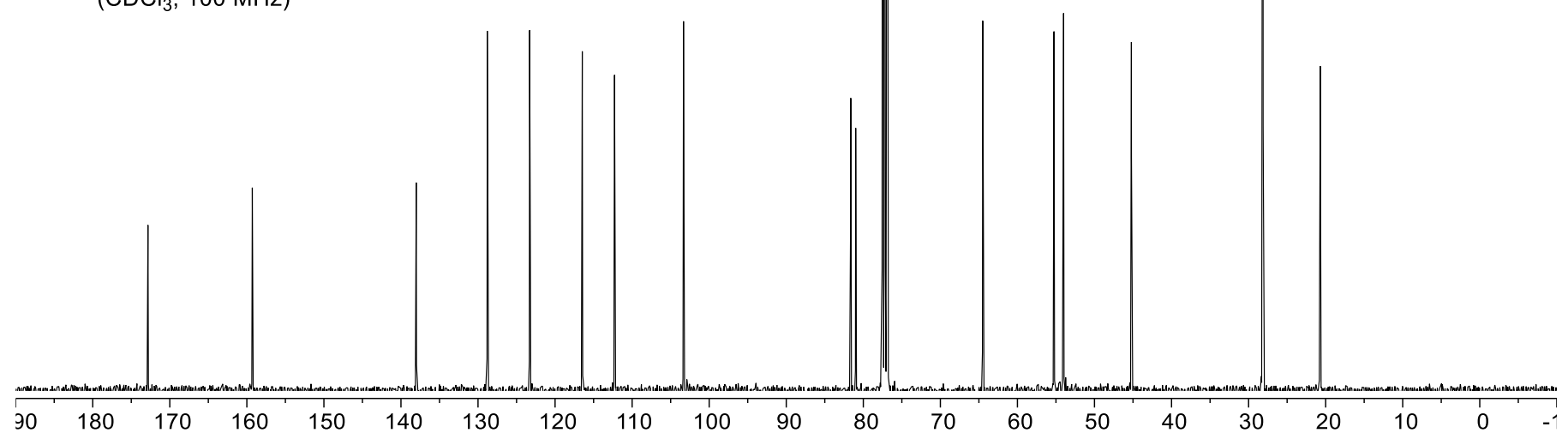


<smiles>CCCOC(=O)C(C)(Cc1ccc2c(c1)OCO2)OCC(C)C</smiles>

$6 \mathbf{a}$

$\left(\mathrm{CDCl}_{3}, 400 \mathrm{MHz}\right)$

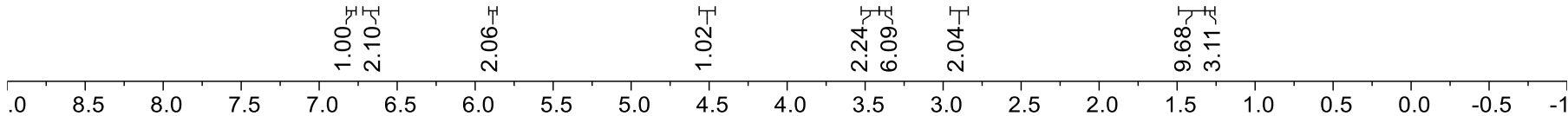<smiles>CCOC(=O)C(C)(Cc1ccc2c(c1)OCO2)OCC(C)C</smiles>

$\left(\mathrm{CDCl}_{3}, 100 \mathrm{MHz}\right)$

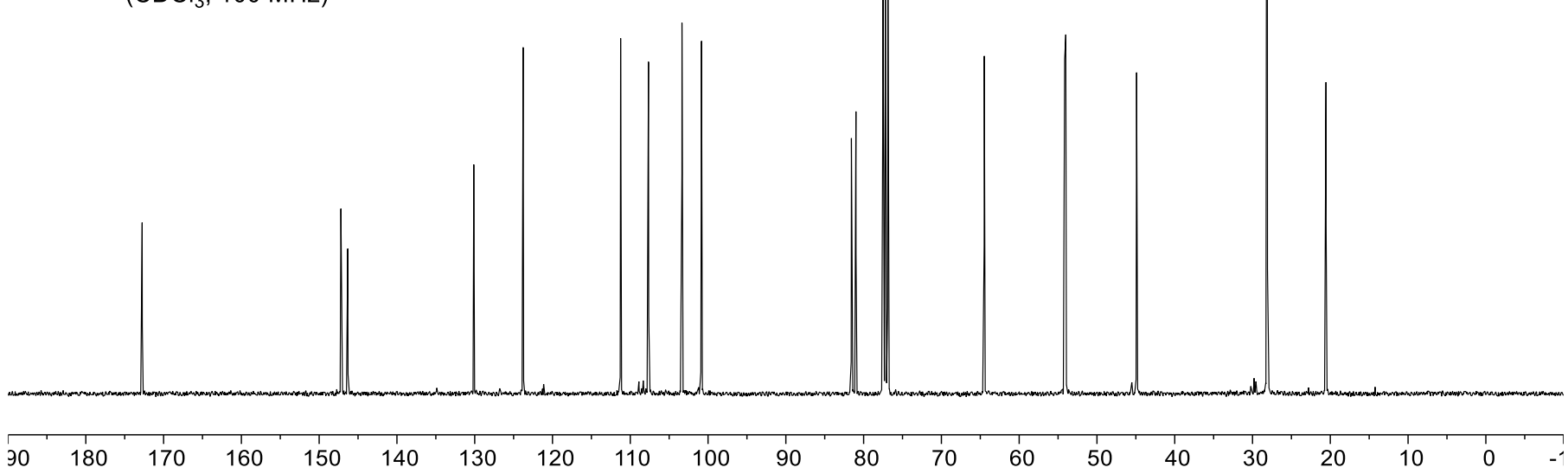




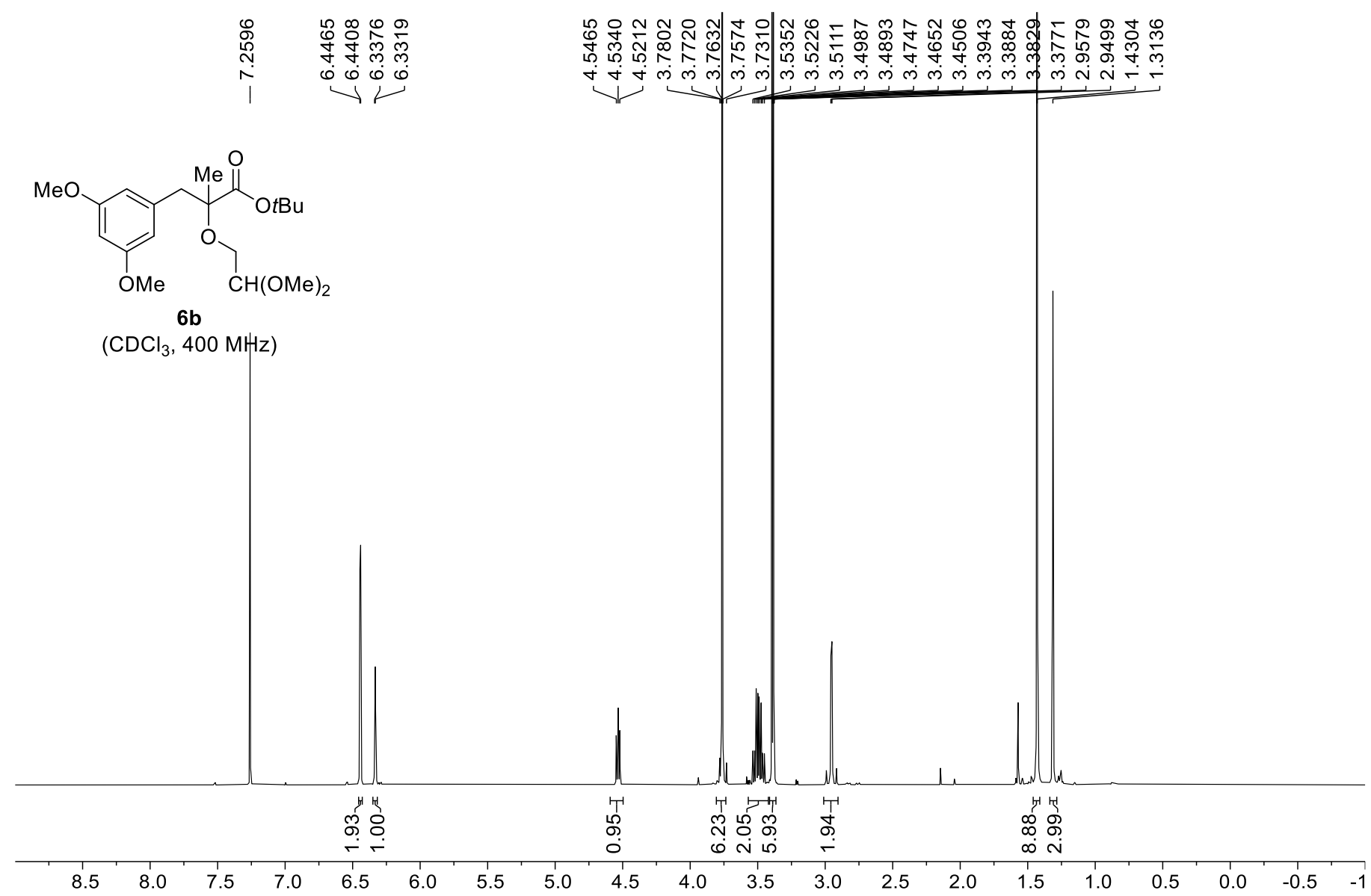

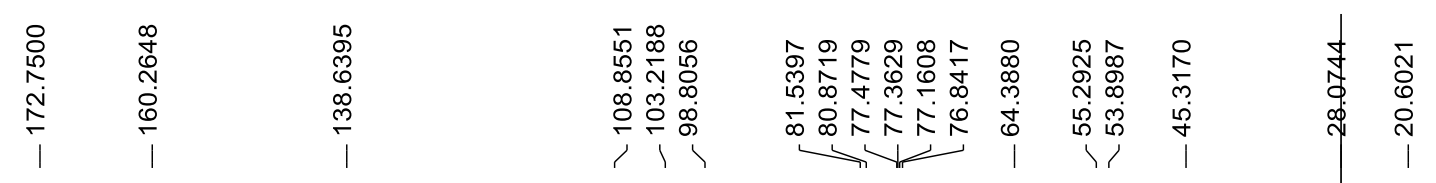<smiles>CCCC(=O)C(C)(Cc1cc(OC)cc(OC)c1)OCC(C)C</smiles>

6b

$\left(\mathrm{CDCl}_{3}, 100 \mathrm{MHz}\right)$

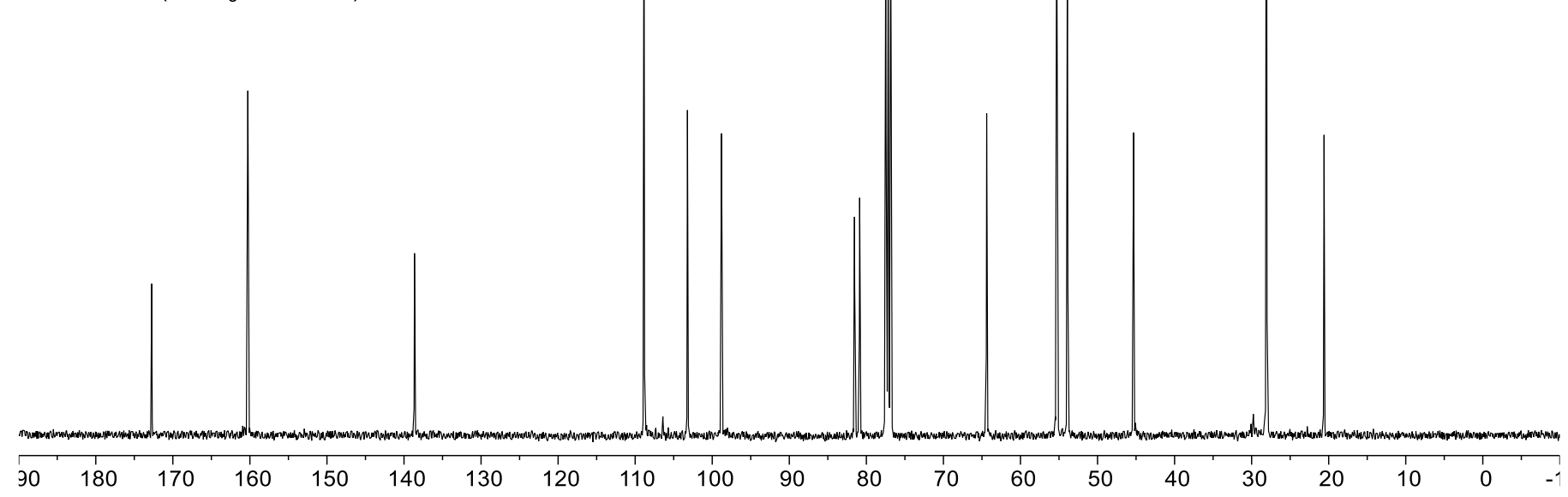


<smiles>CCOC(=O)C(C)(Cc1ccc(F)c(OC)c1)OCC(C)C</smiles>

$6 c$

$\left(\mathrm{CDCl}_{3}, 400 \mathrm{MHz}\right)$

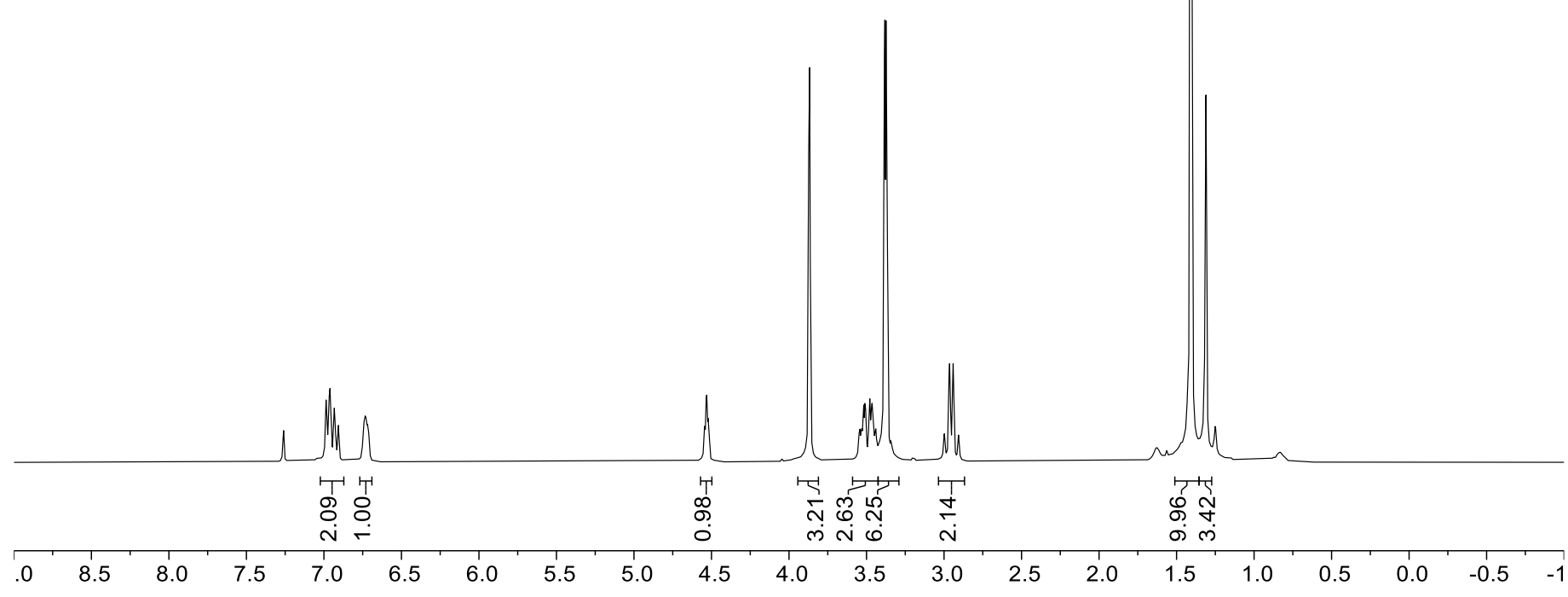<smiles>CCOC(=O)C(C)(Cc1ccc(F)c(O)c1)OCC(C)O</smiles>

6c

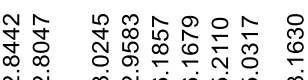

$\left(\mathrm{CDCl}_{3}, 100 \mathrm{MHz}\right)$

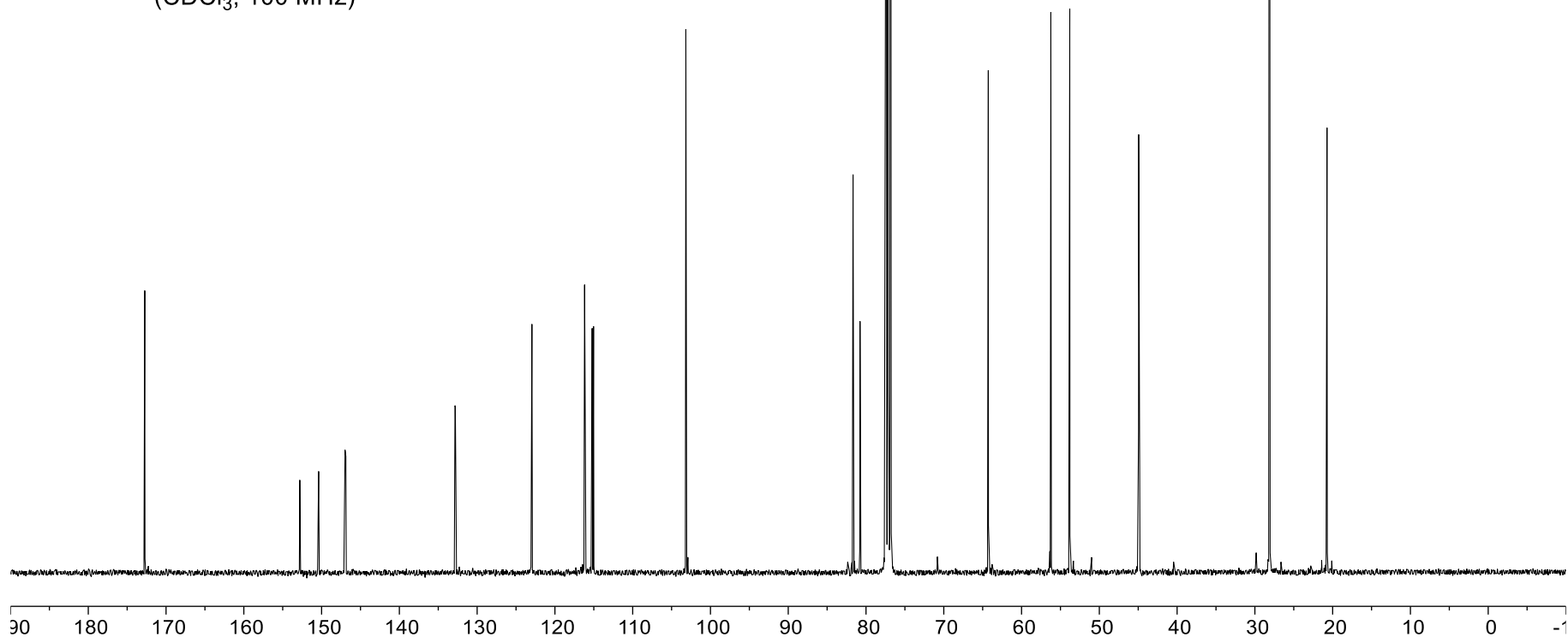


<smiles>CCOC(=O)C(C)(Cc1cccc(C)c1)OCCC(C)C</smiles>

$\left(\mathrm{CDCl}_{3}, 400 \mathrm{MHz}\right)$

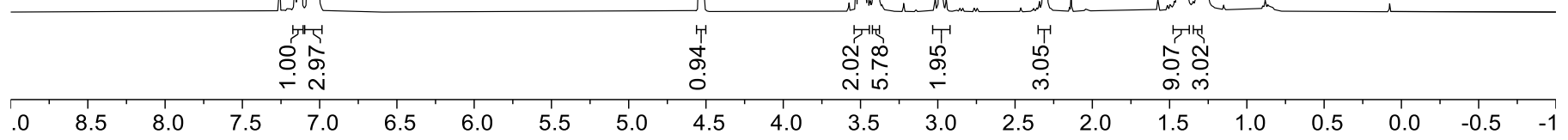

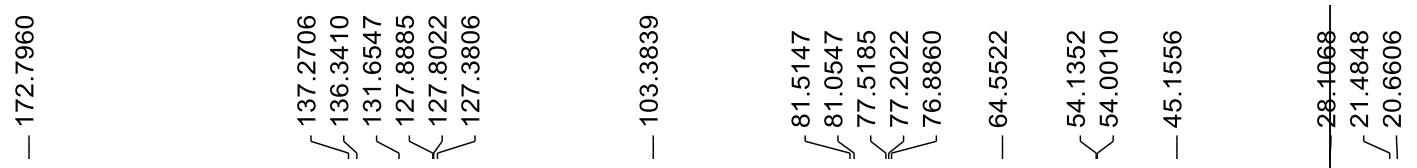<smiles>CCCCOC(=O)C(C)(Cc1cccc(C)c1)OCC(C)OC</smiles>
$\left(\mathrm{CDCl}_{3}, 100 \mathrm{MHz}\right)$
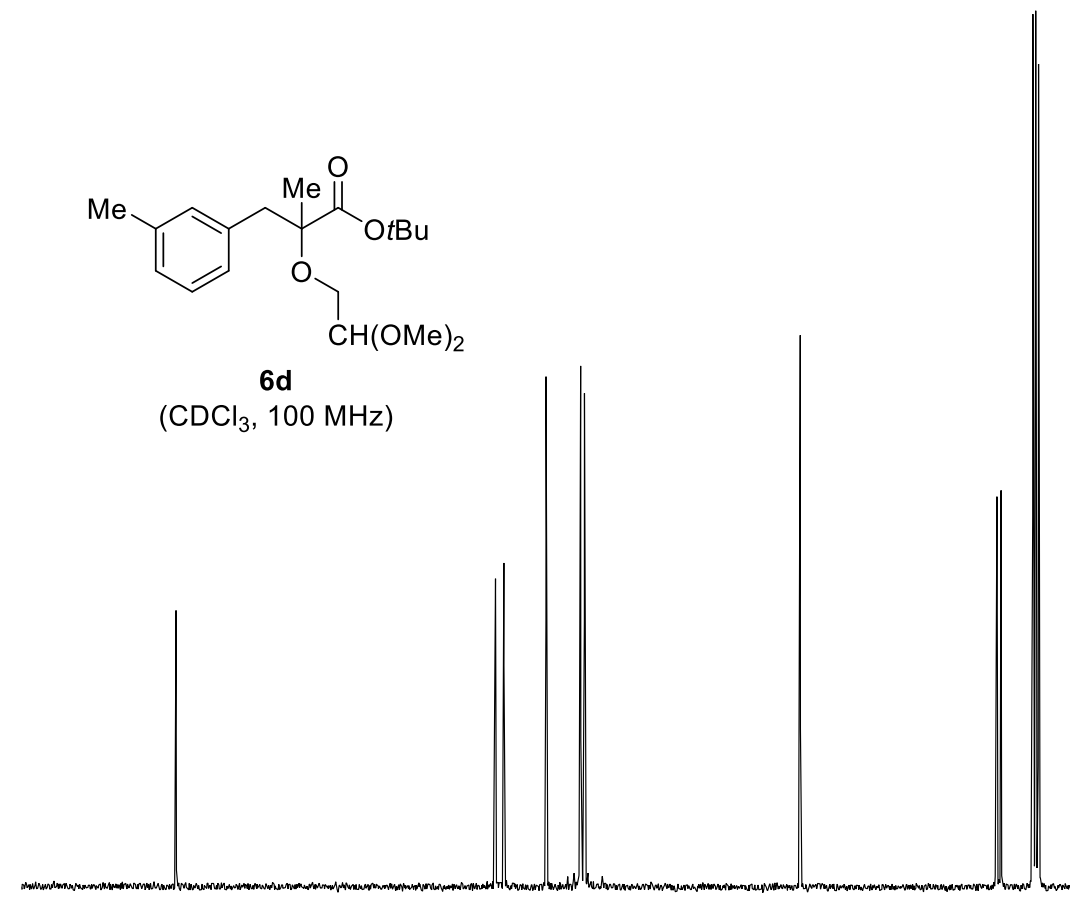
<smiles>CCCOC(=O)C(C)(Cc1ccccc1)OCC(C)C</smiles>

$\left(\mathrm{CDCl}_{3}, 400 \mathrm{MHz}\right)$

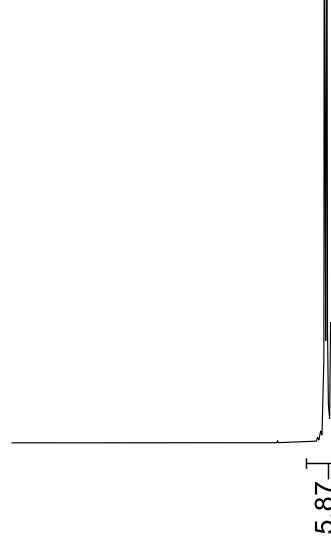

\begin{tabular}{lllllllll}
\hline 0 & 8.5 & 8.0 & 7.5 & 7.0 & 6.5 & 6.0 & 5.5 & 5.0
\end{tabular}

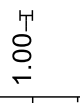

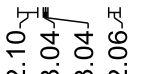

守皆

$\begin{array}{lllll}3.5 & 30 & 25 & 20 & 15\end{array}$

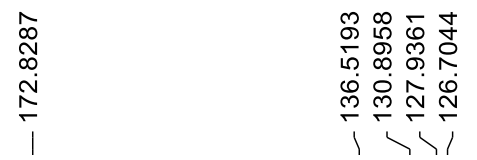

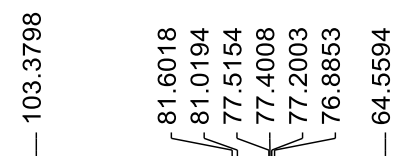

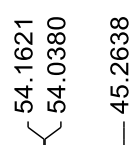

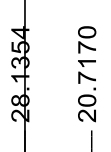<smiles>CCCOC(=O)C(C)(Cc1ccccc1)OCC(C)C</smiles>

$\left(\mathrm{CDCl}_{3}, 100 \mathrm{MHz}\right)$

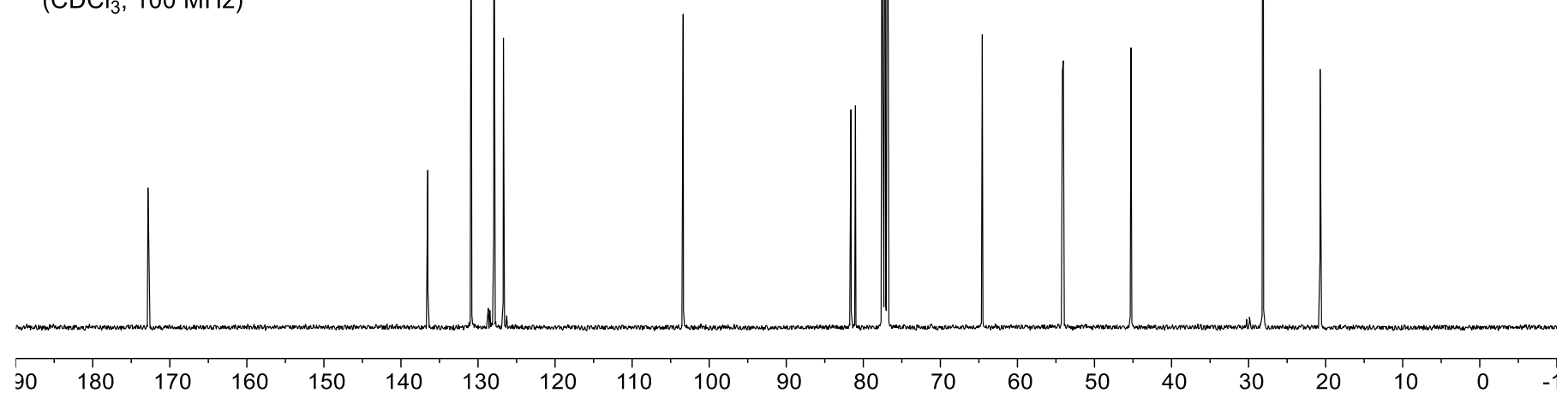




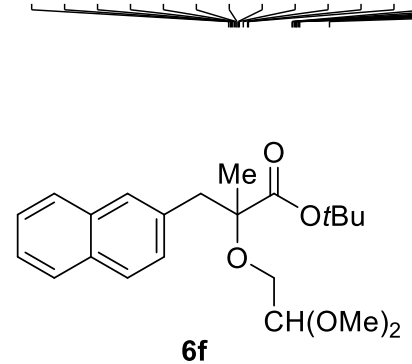

$\left(\mathrm{CDCl}_{3}, 400 \mathrm{MHz}\right)$
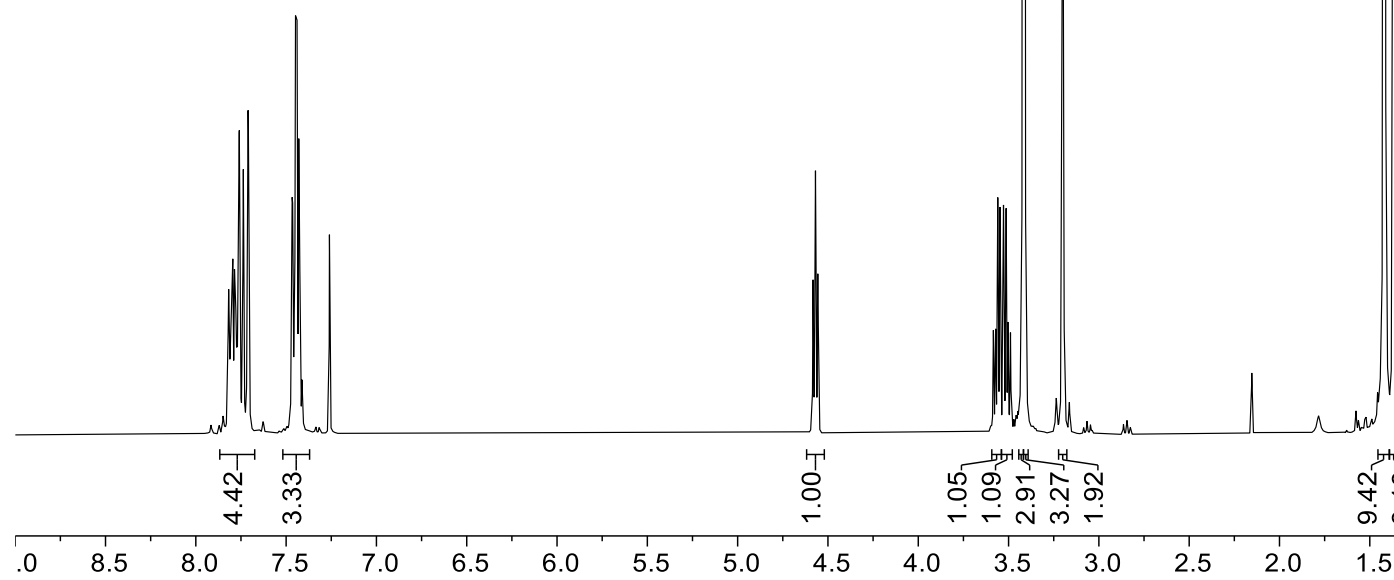

\section{\%"th}

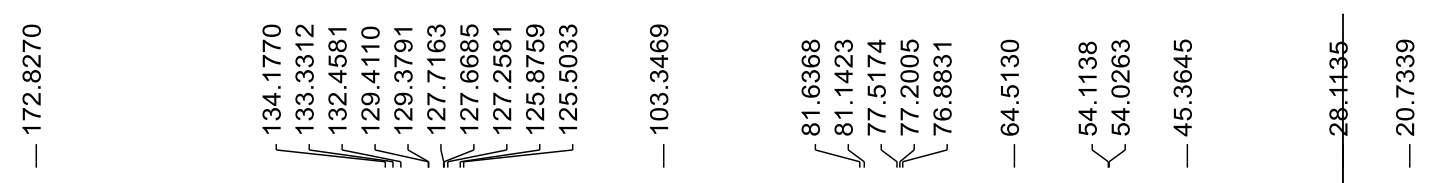

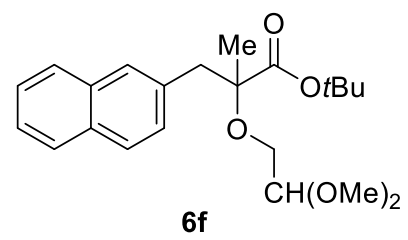

$\left(\mathrm{CDCl}_{3}, 100 \mathrm{MHz}\right)$

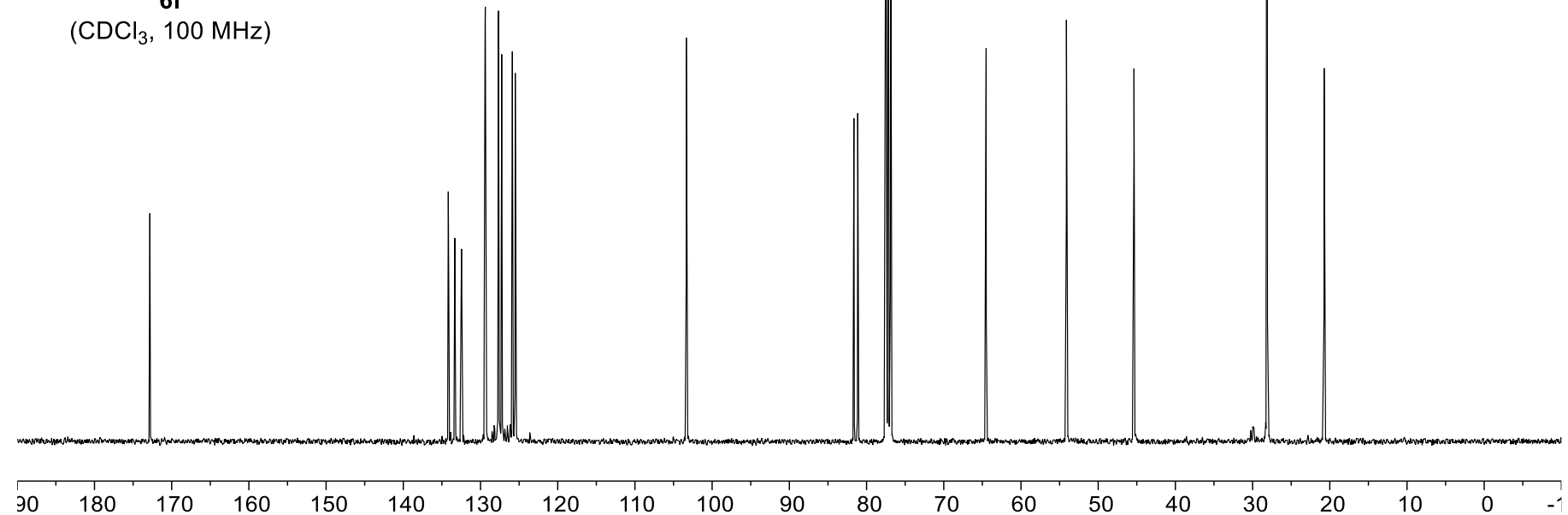


<smiles>CCOC(=O)C(C)(Cc1cccc(Br)c1)OCCC(C)C</smiles>

$6 \mathrm{~g}$

$\left(\mathrm{CDCl}_{3}, 400 \mathrm{MHz}\right)$
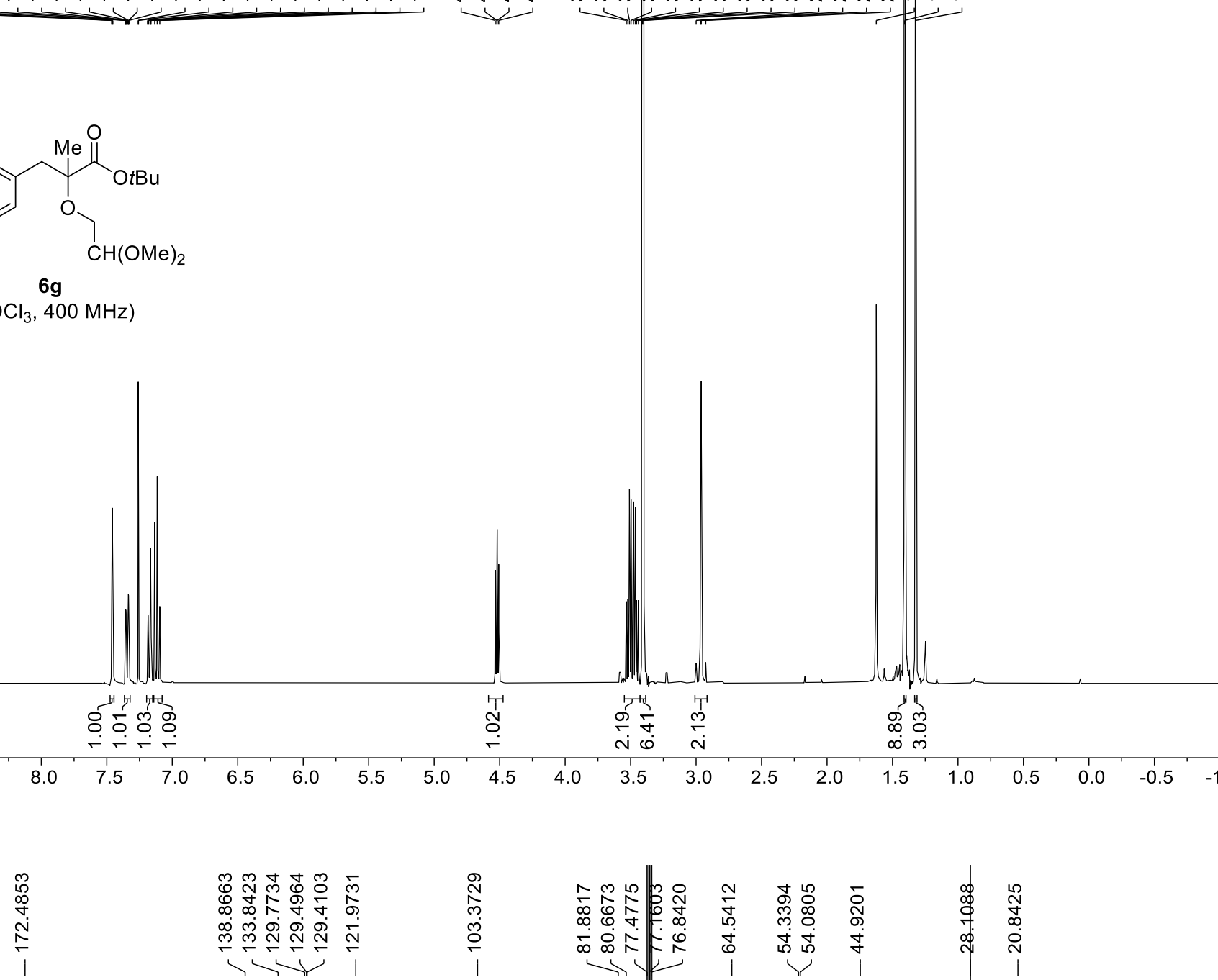<smiles>CCCOC(=O)C(C)(Cc1cccc(Br)c1)OCCC(C)C</smiles>

$$
6 \mathbf{g}
$$

$\left(\mathrm{CDCl}_{3}, 100 \mathrm{MHz}\right)$

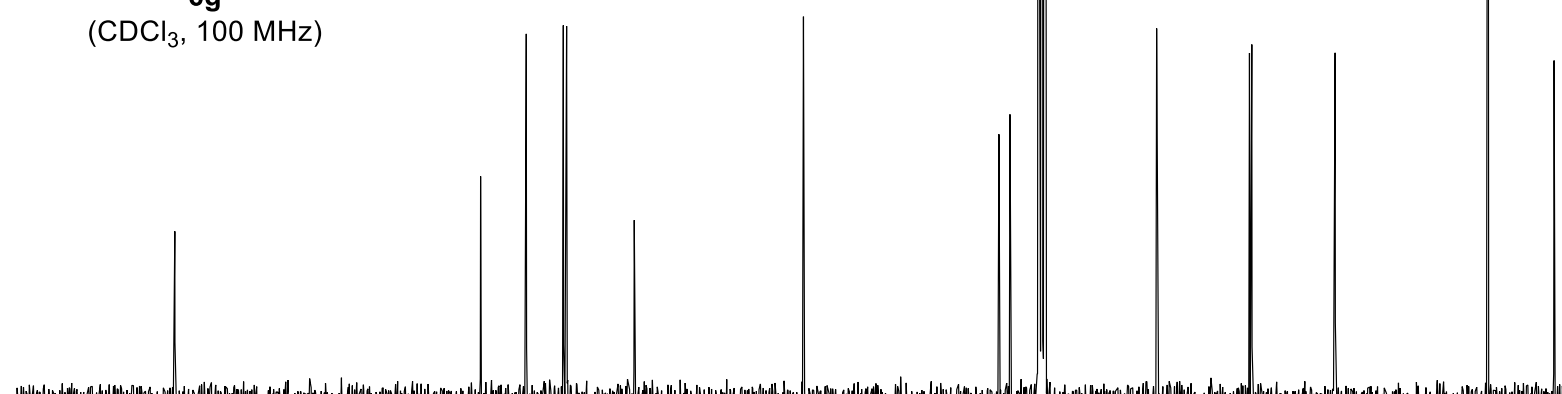




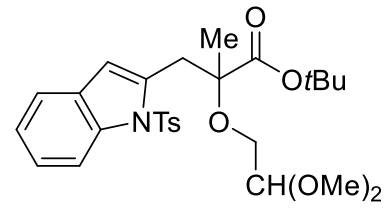

$6 h$

$\left(\mathrm{CDCl}_{3}, 400 \mathrm{MHz}\right)$
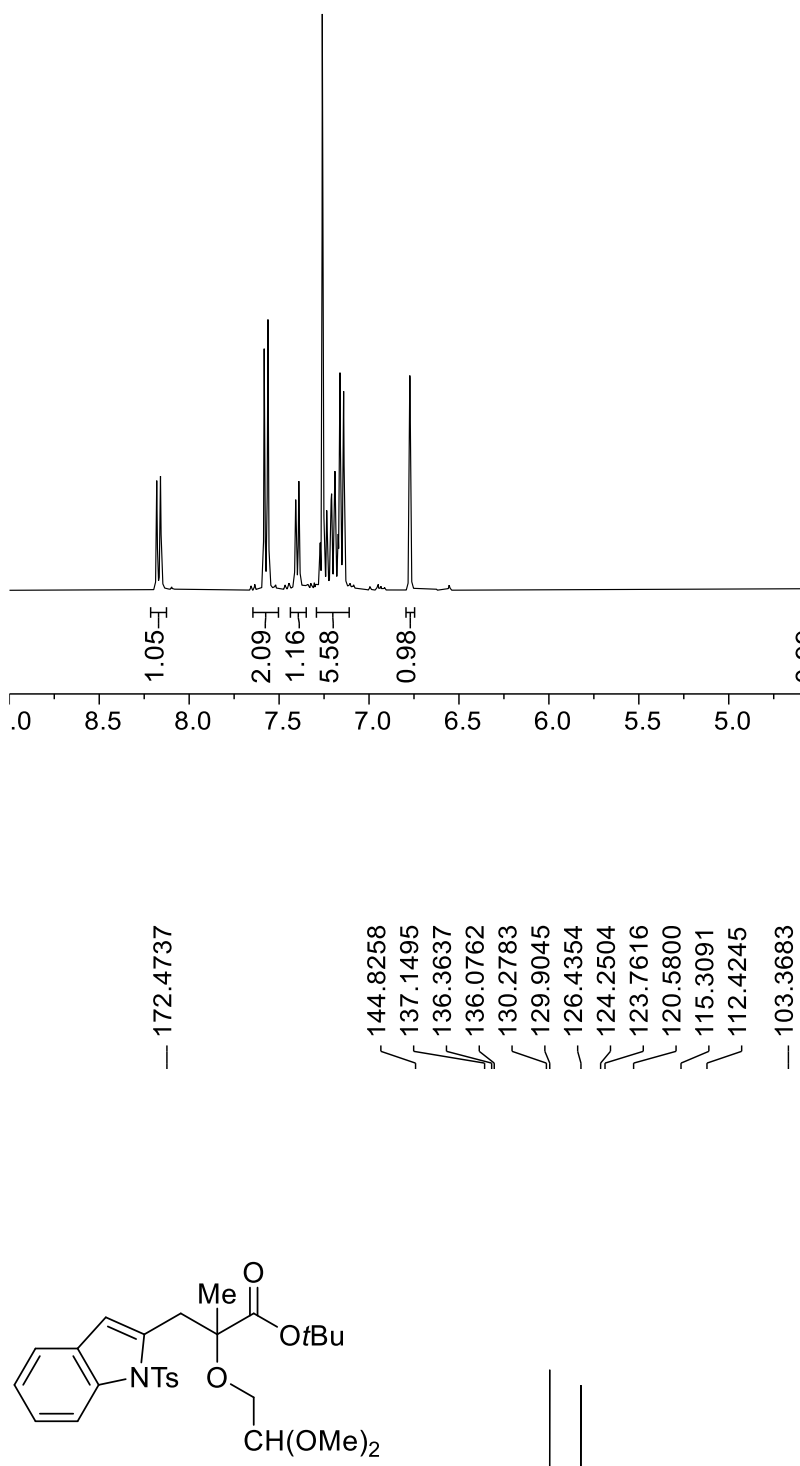

$6 h$

$\left(\mathrm{CDCl}_{3}, 100 \mathrm{MHz}\right)$
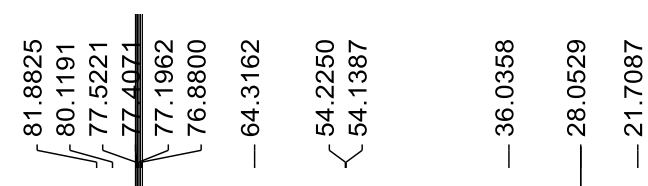

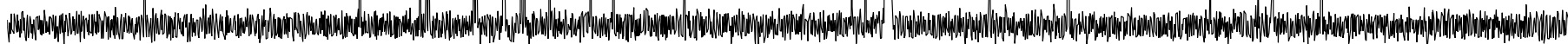

\begin{tabular}{|c|}
\hline $180 \quad 17$ \\
\hline
\end{tabular}




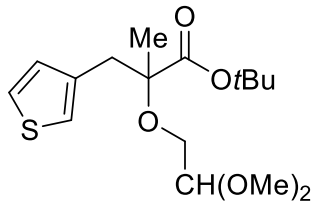

$6 i$

$\left(\mathrm{CDCl}_{3}, 400 \mathrm{MHz}\right)$
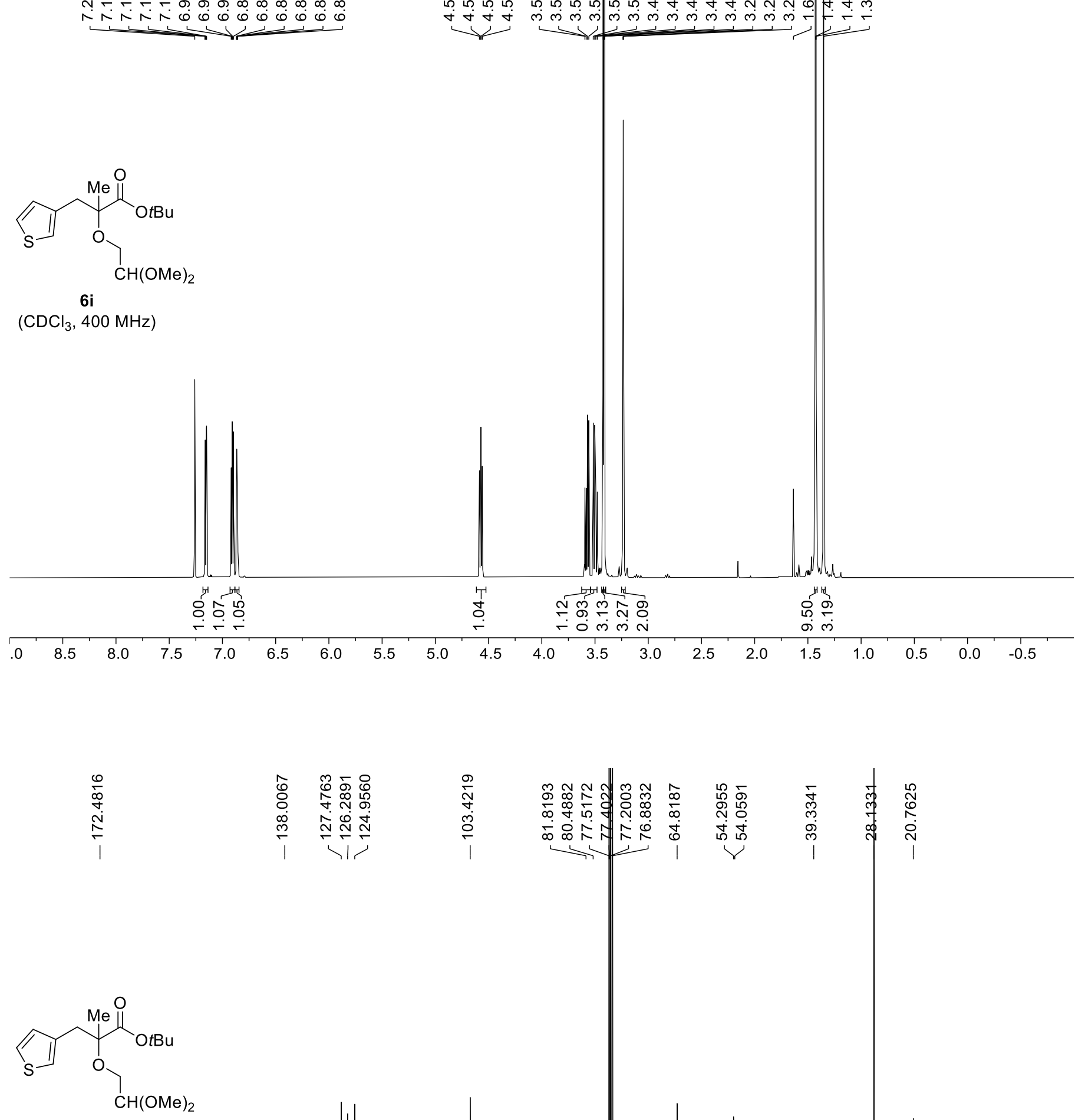

$\left(\mathrm{CDCl}_{3}, 100 \mathrm{MHz}\right)$

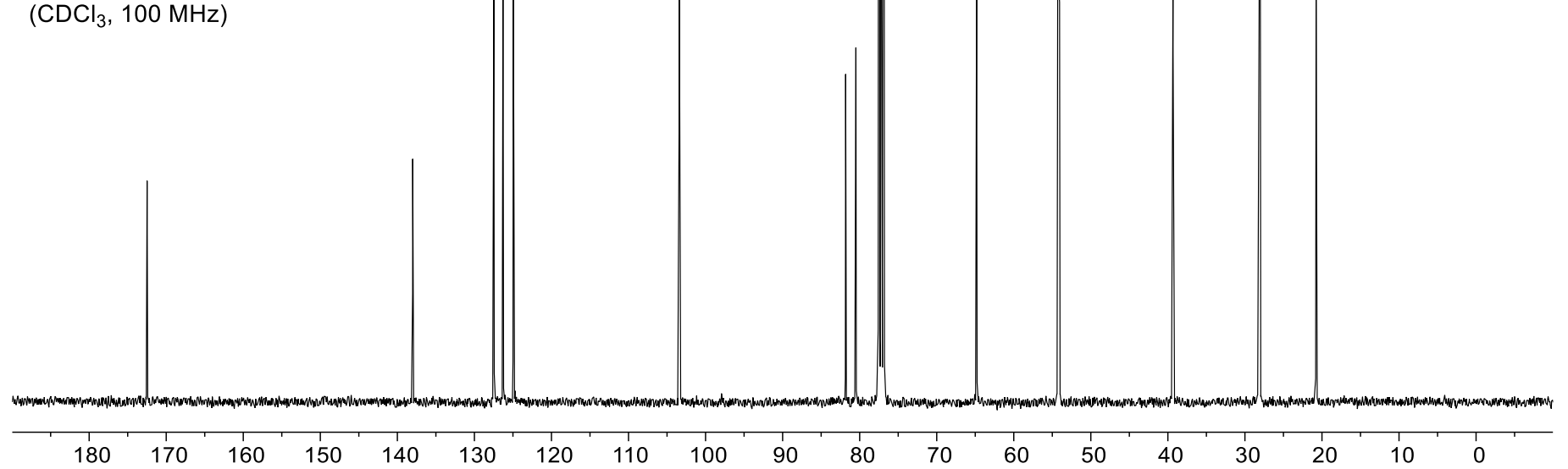




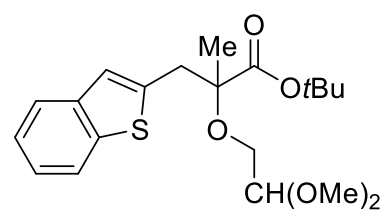

$6 \mathbf{j}$

$\left(\mathrm{CD}_{3} \mathrm{OD}, 400 \mathrm{MHz}\right)$
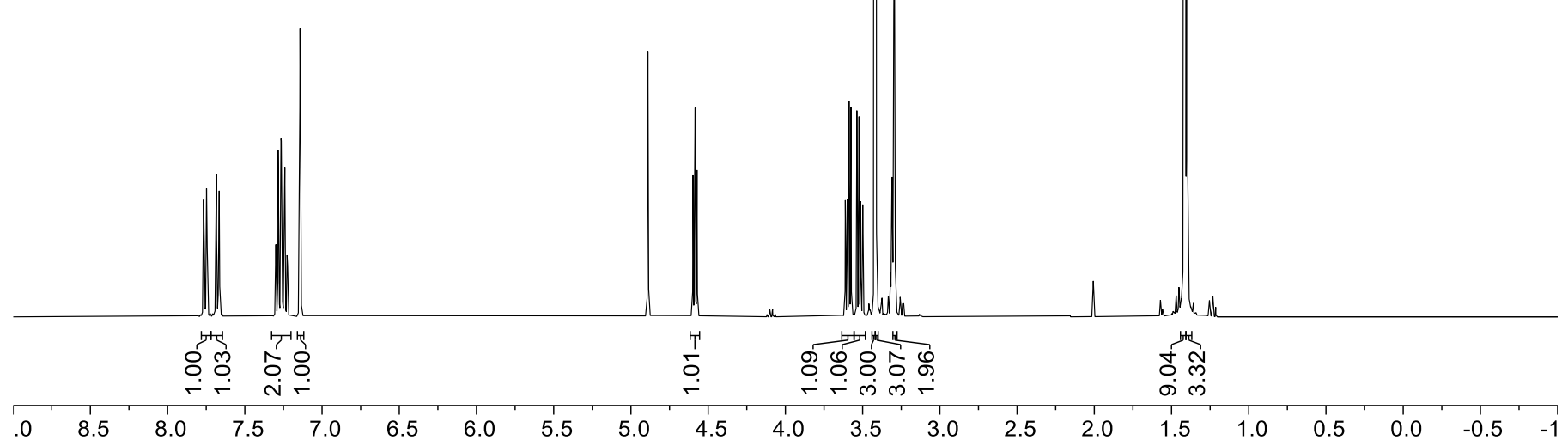

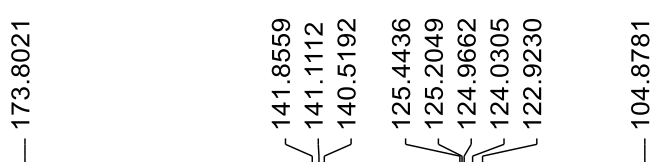

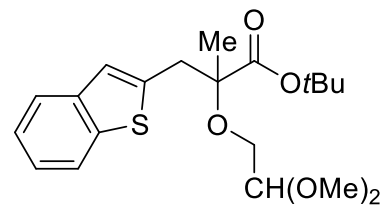

6j

$\left(\mathrm{CD}_{3} \mathrm{OD}, 100 \mathrm{MHz}\right)$

\section{$\infty$
$\infty$
0
$\dot{0}$
$\dot{0}$}

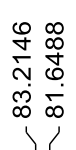
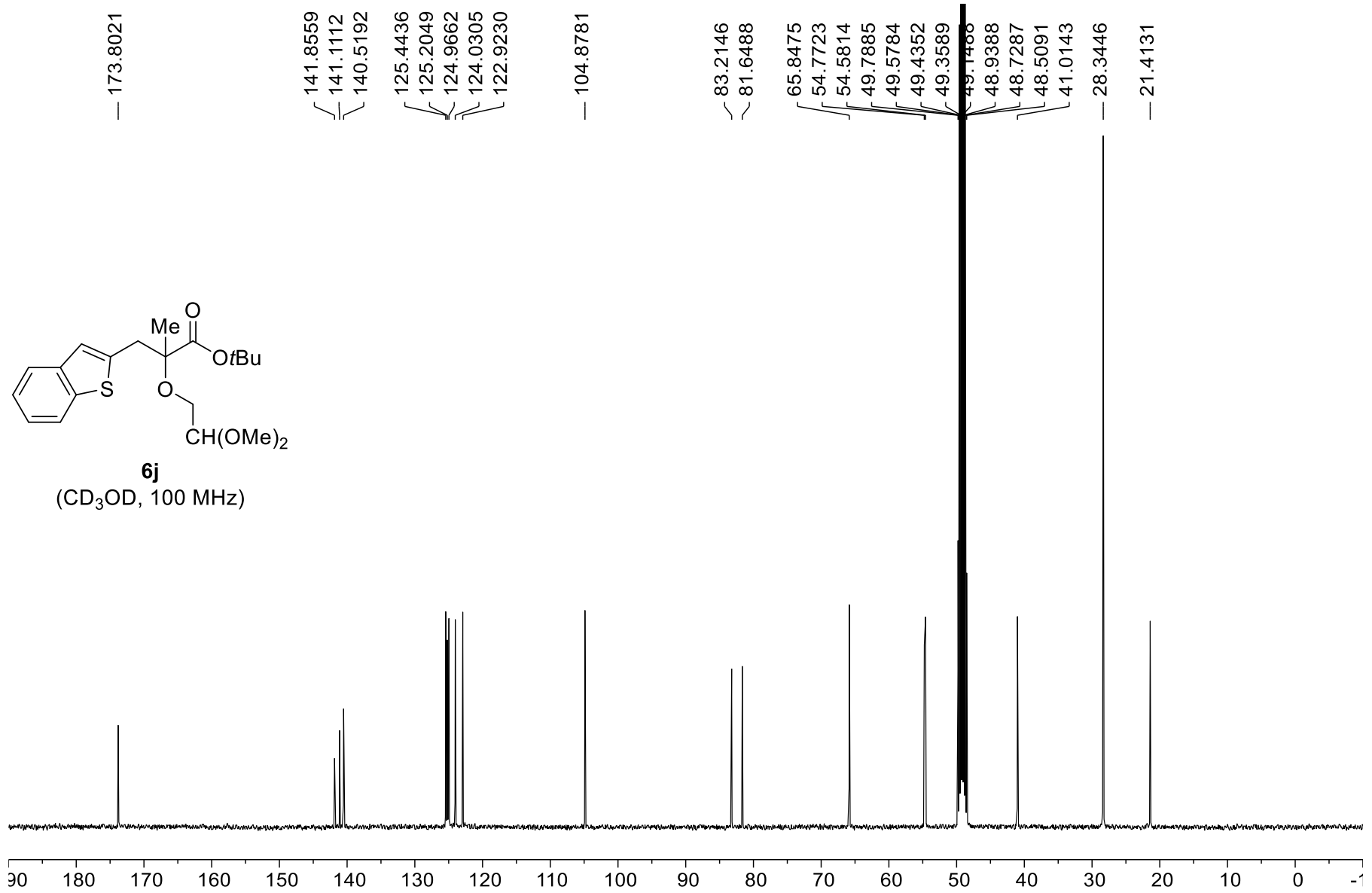


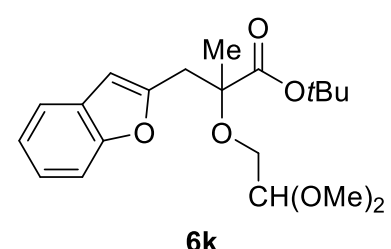

$\left(\mathrm{CDCl}_{3}, 400 \mathrm{MHz}\right)$

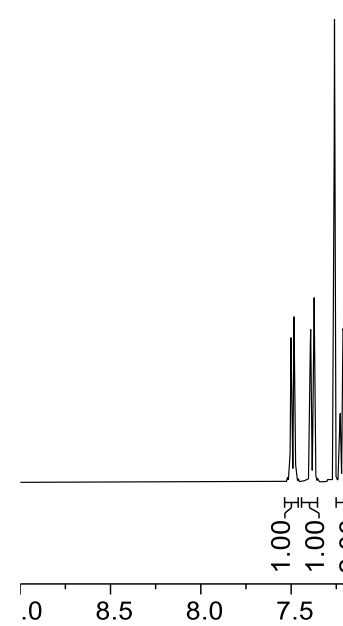

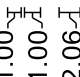

\section{तो}
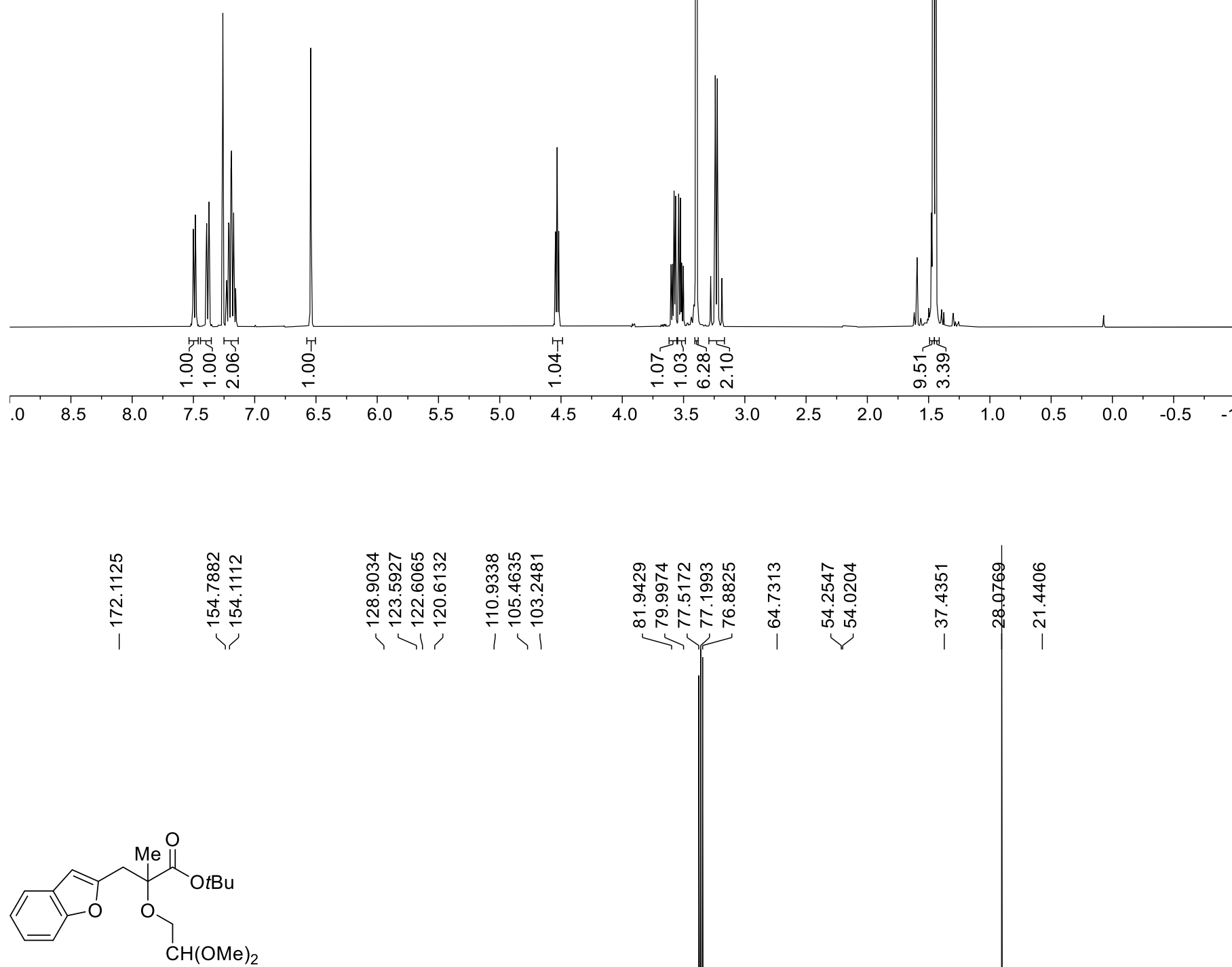

6k

$\left(\mathrm{CDCl}_{3}, 100 \mathrm{MHz}\right)$

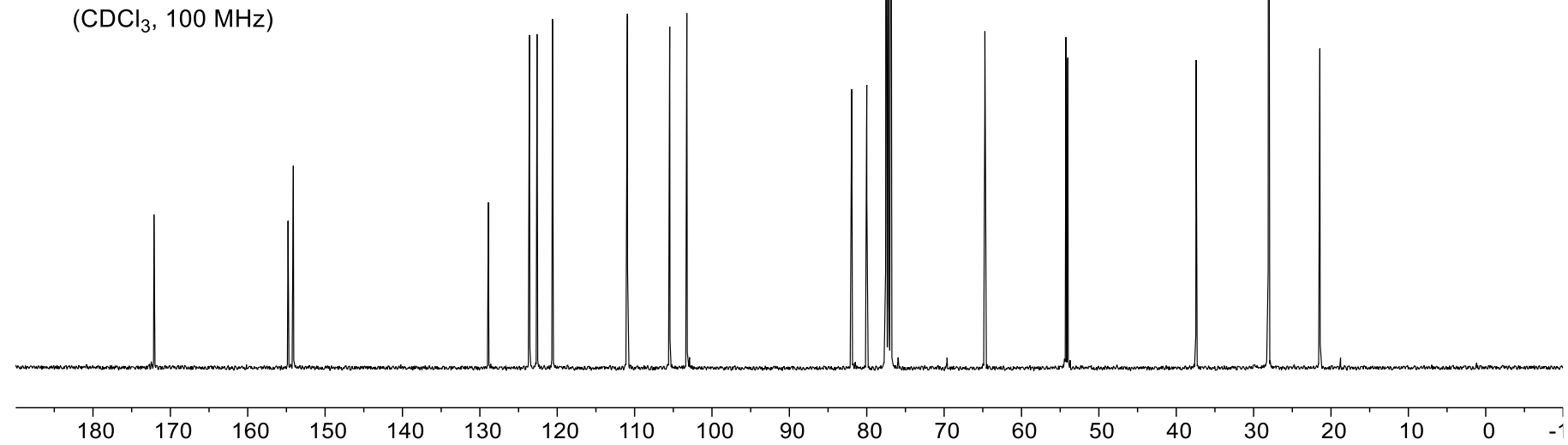




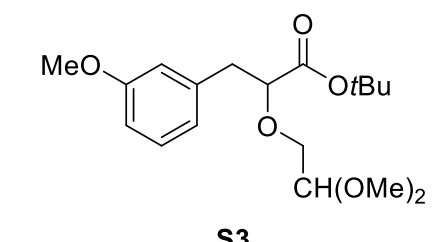

S3

$\left(\mathrm{CDCl}_{3}, 400 \mathrm{MHz}\right)$

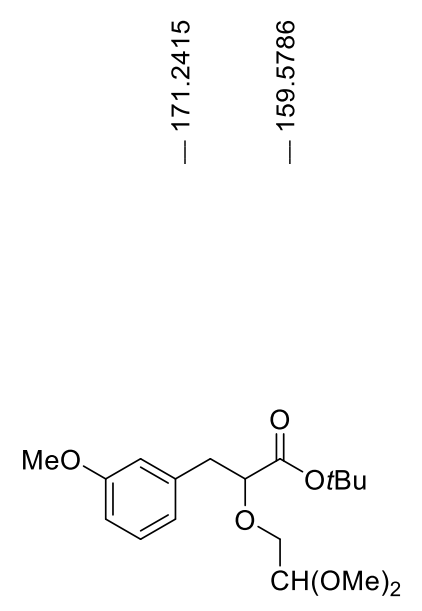

S3

$\left(\mathrm{CDCl}_{3}, 100 \mathrm{MHz}\right)$

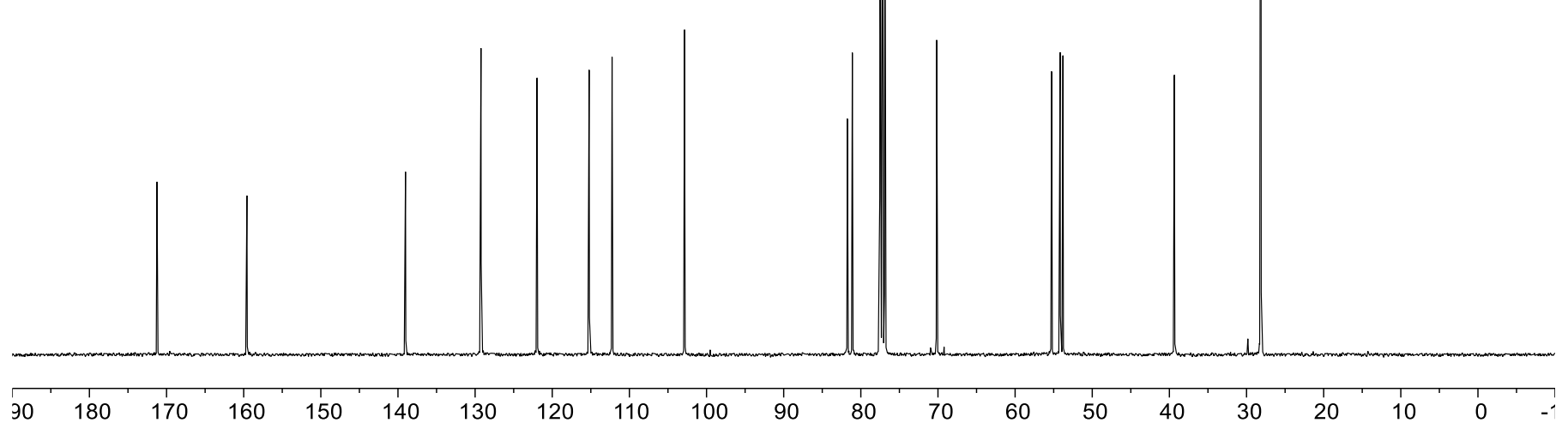


<smiles>CCCOC(=O)C(Cc1ccccc1)(Cc1cccc(OC)c1)OCC(C)C</smiles>

61

$\left(\mathrm{CDCl}_{3}, 400 \mathrm{MHz}\right)$

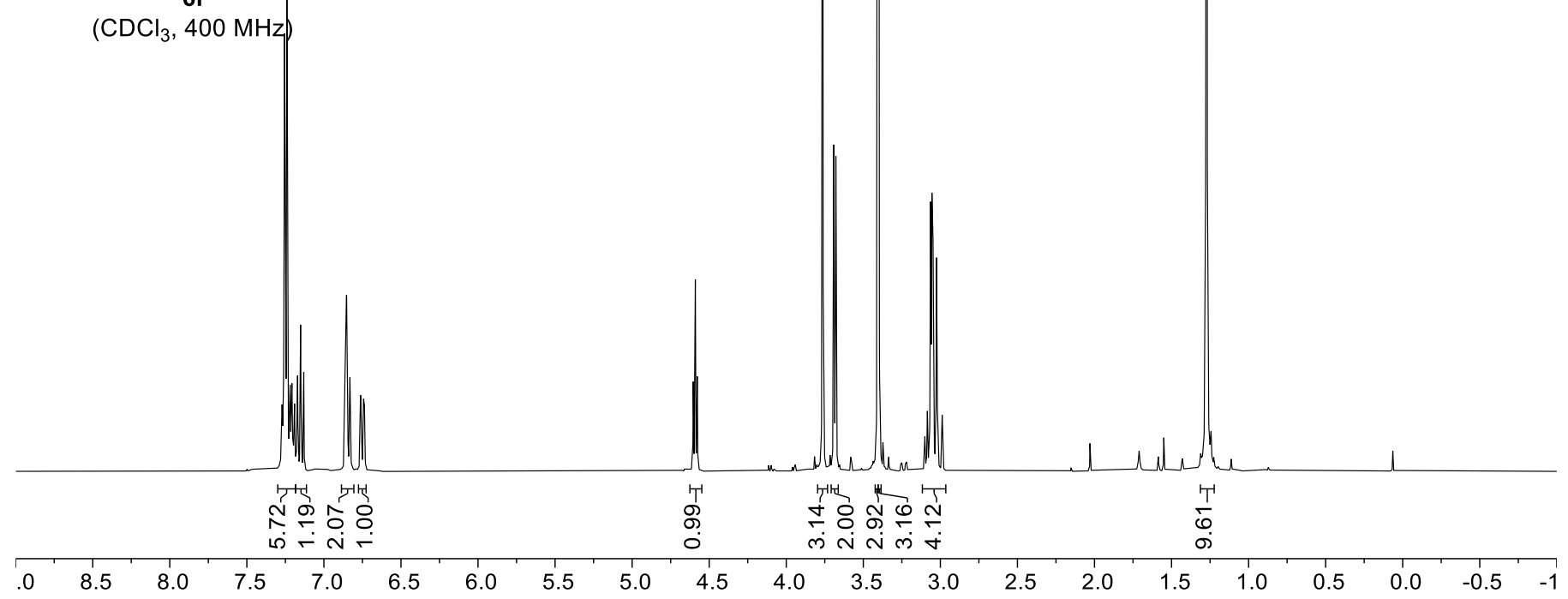

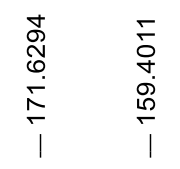

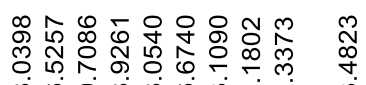

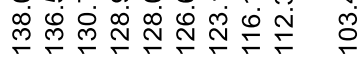

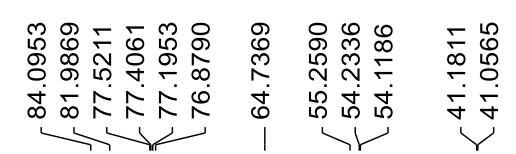

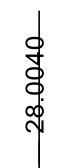<smiles>CCCCOC(Cc1ccccc1)(Cc1cccc(OC)c1)C(=O)OCC(C)C</smiles>

6I

$\left(\mathrm{CDCl}_{3}, 100 \mathrm{MHz}\right)$

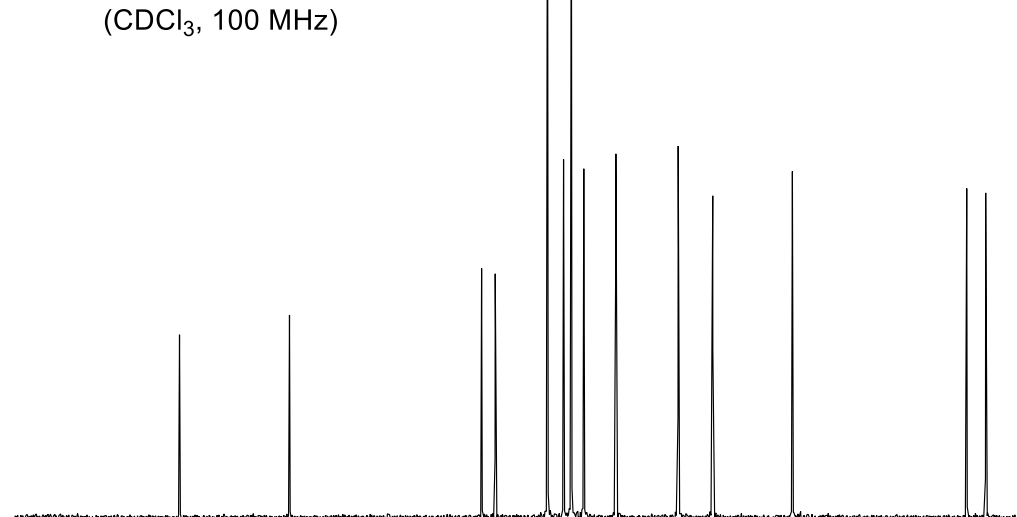

90

180

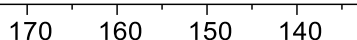

130

$\begin{array}{lll}120 & 110 \quad 100\end{array}$
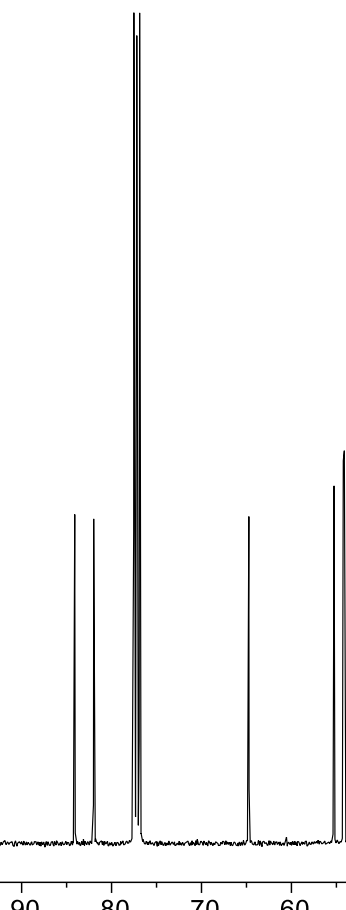
<smiles>C=CCC(Cc1cccc(OC)c1)(OCC(C)C)C(=O)OCC</smiles>

$6 \mathrm{~m}$

$\left(\mathrm{CD}_{3} \mathrm{OD}, 400 \mathrm{MHz}\right)$ $\begin{array}{ll}1 & 8 \\ 0 & 0 \\ 0 & \infty \\ 0 & 8 \\ & 0 \\ 1 & 1\end{array}$

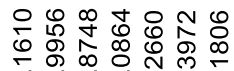

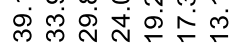

i<smiles>C=CCC(Cc1cccc(OC)c1)(OCC)C(=O)OCC</smiles>

$6 \mathrm{~m}$

$\left(\mathrm{CD}_{3} \mathrm{OD}, 100 \mathrm{MHz}\right)$

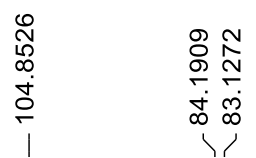

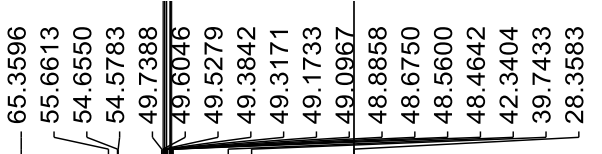



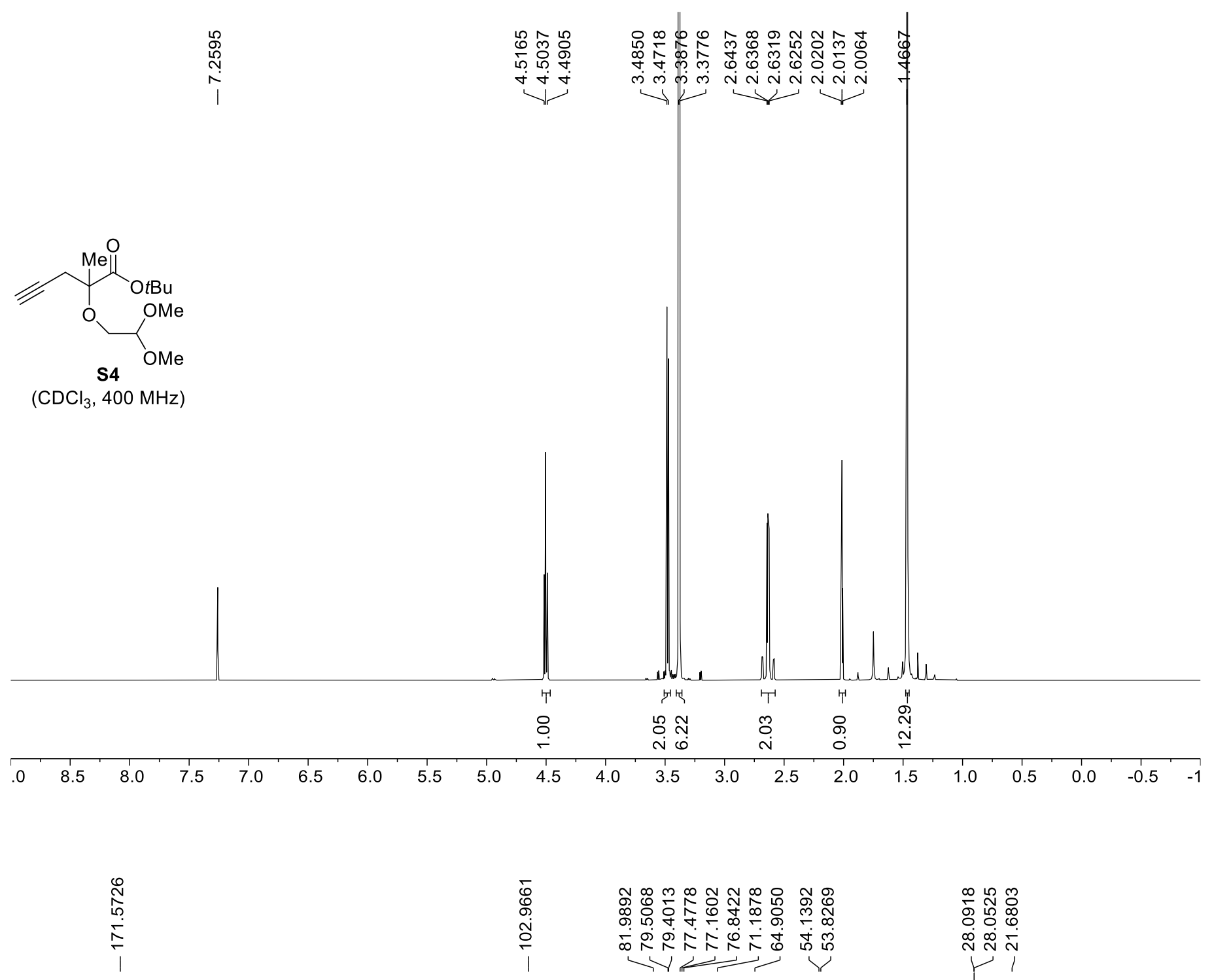

冓

$\stackrel{\infty}{\sim} \stackrel{\infty}{\sim}$

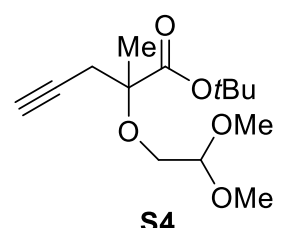

$\left(\mathrm{CDCl}_{3}, 100 \mathrm{MHz}\right)$

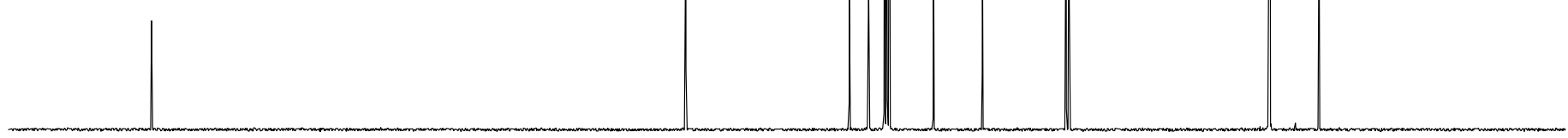




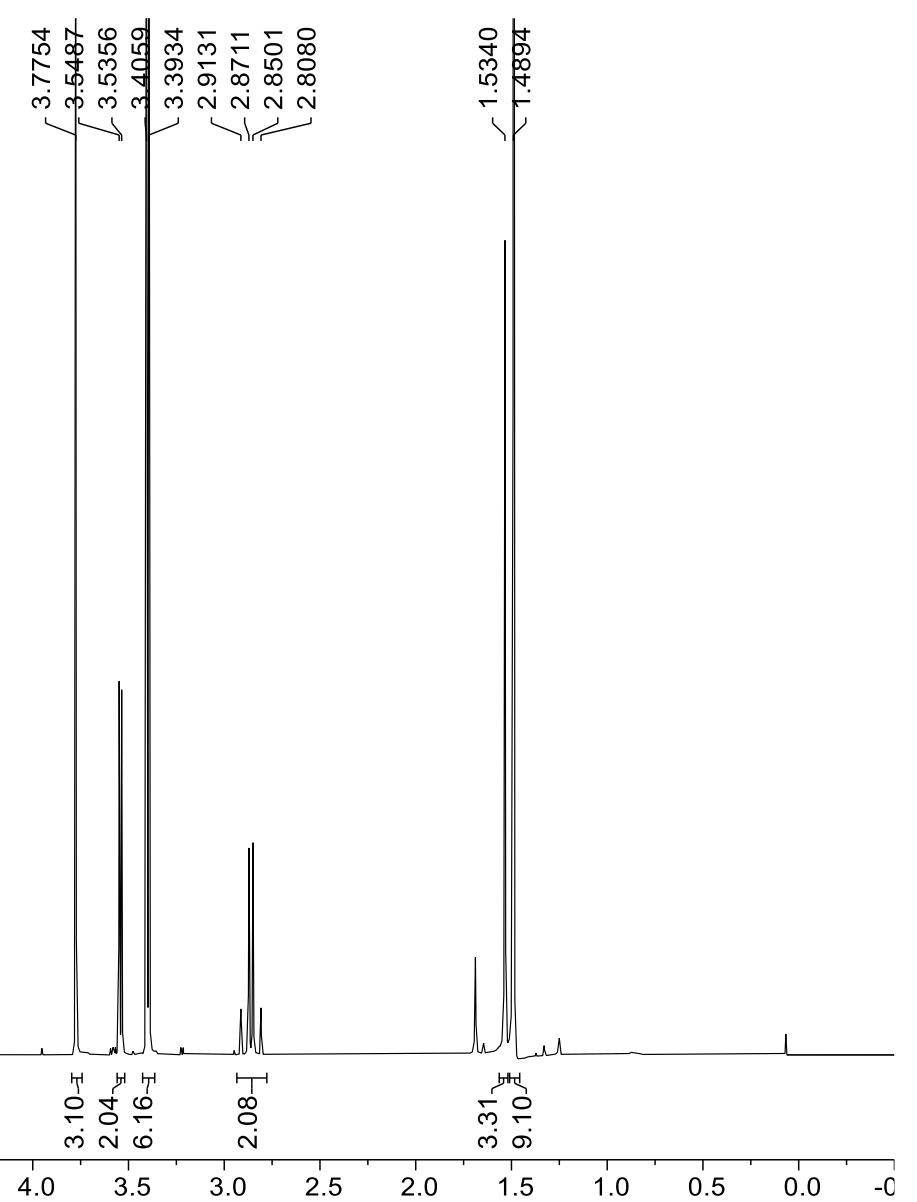

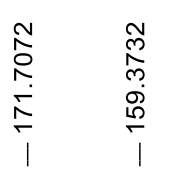

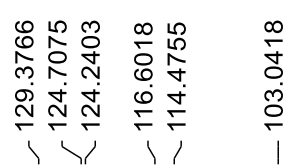

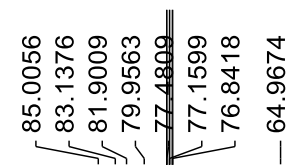

0
0

월동요

ฟ

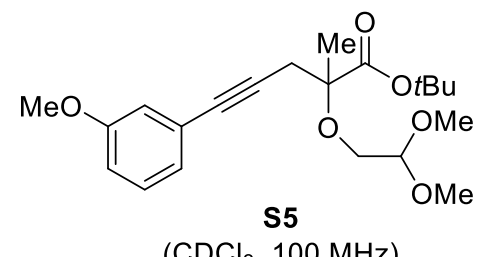

$\left(\mathrm{CDCl}_{3}, 100 \mathrm{MHz}\right)$

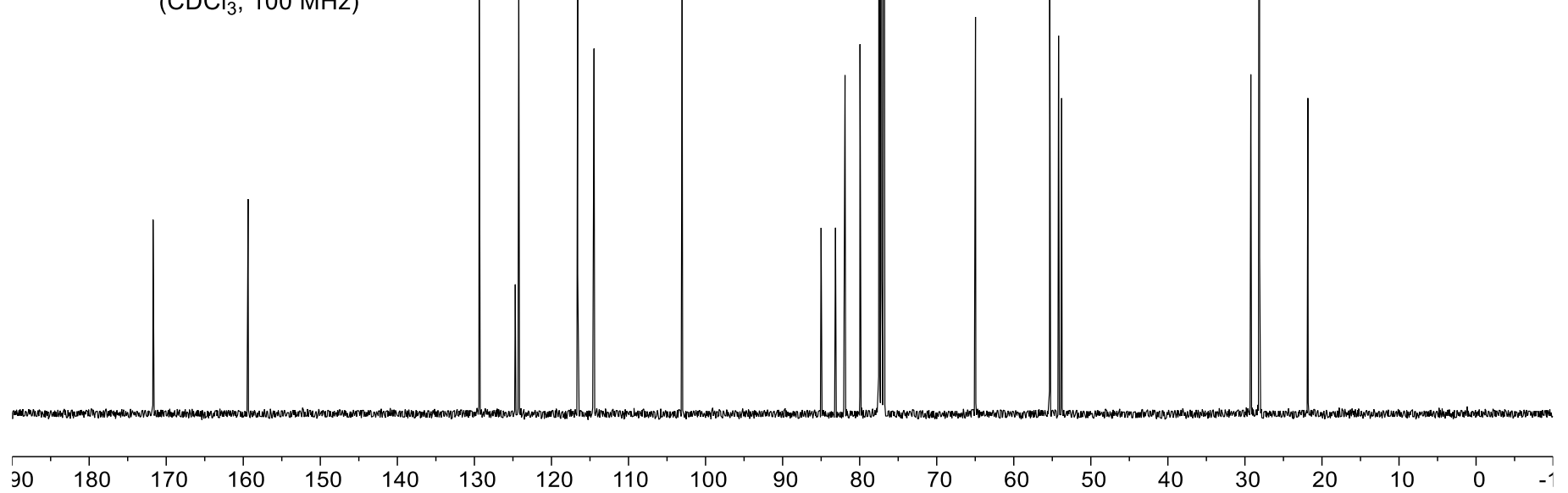




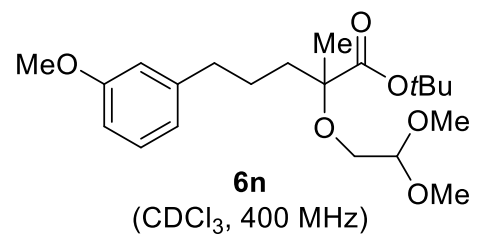

$\mathrm{CDCl}_{3}, 400 \mathrm{MHz}$

$\mathrm{OMe}$

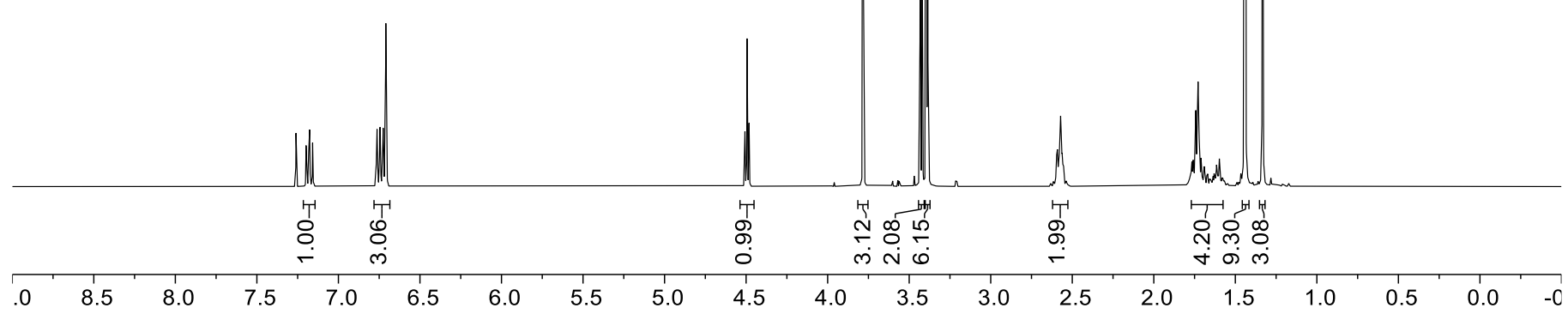

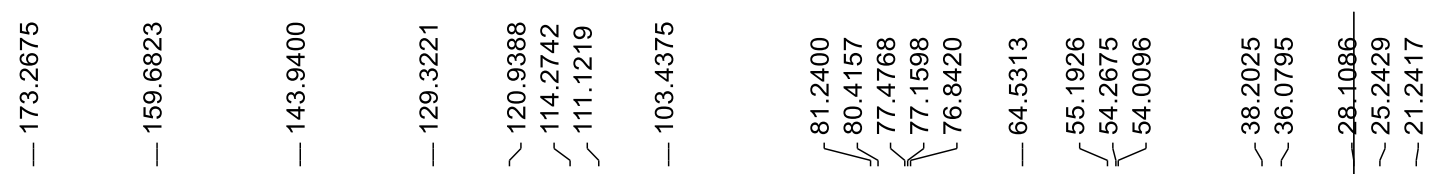
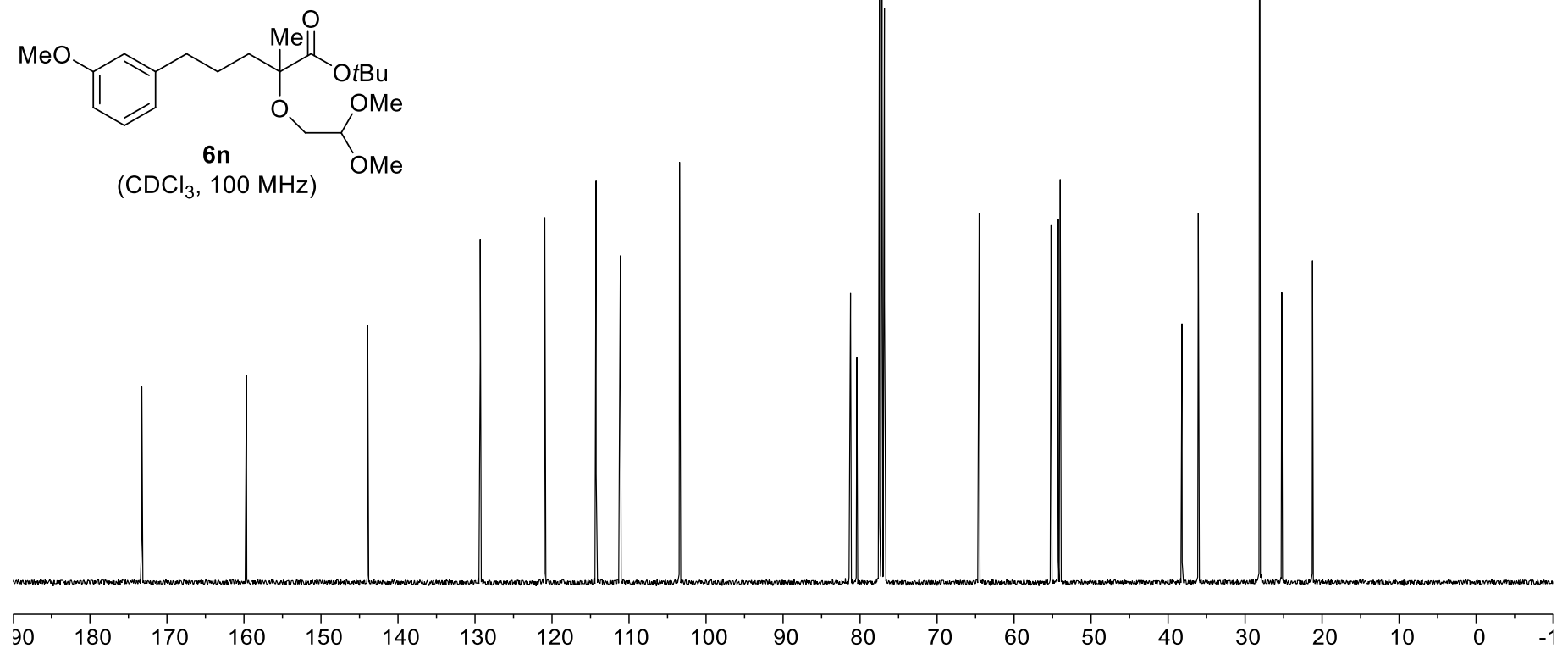


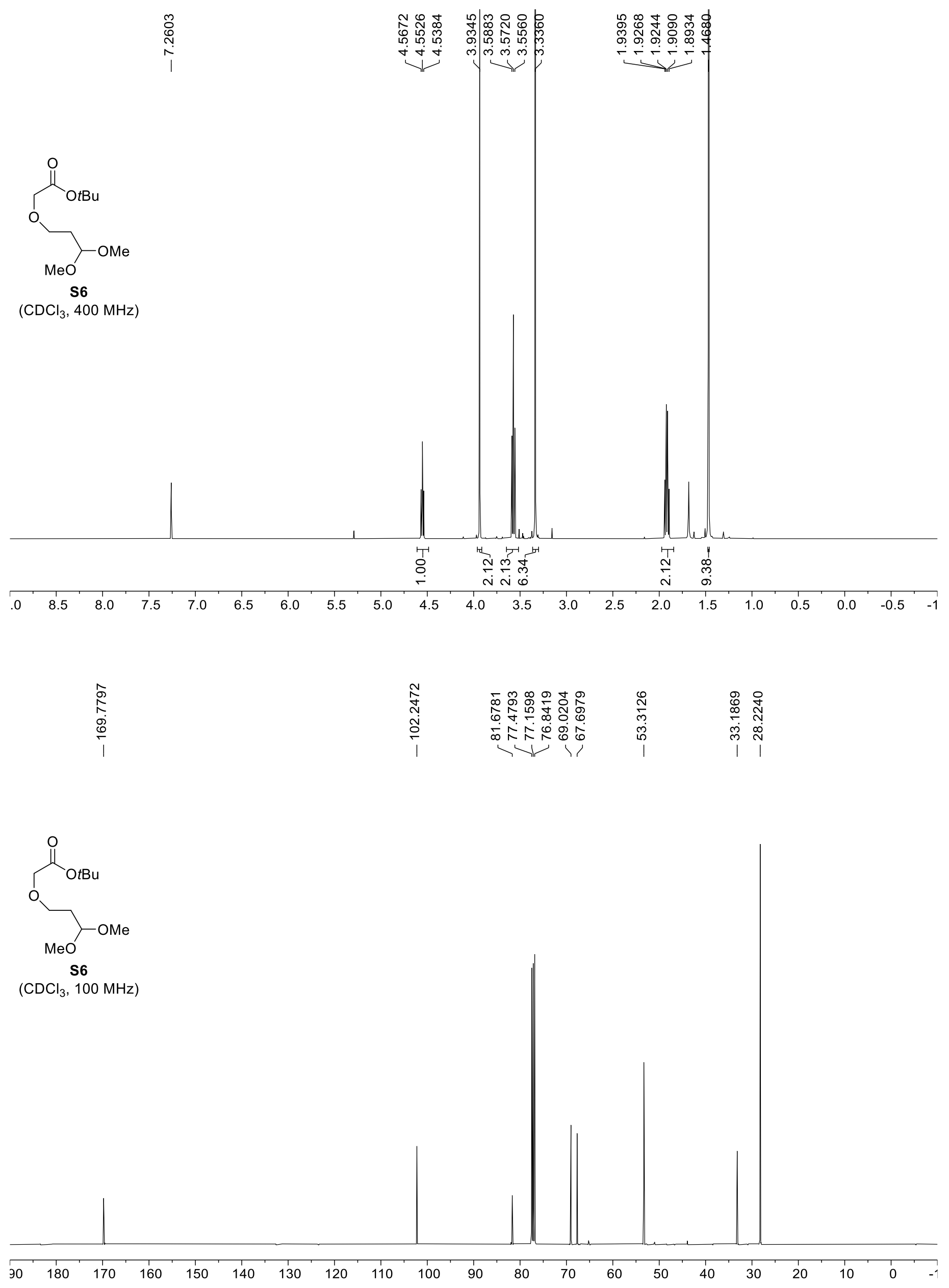


<smiles>CCCCOCC(=O)CCCOC(C)COC</smiles>

S7

$\left(\mathrm{CDCl}_{3}, 400 \mathrm{MHz}\right)$

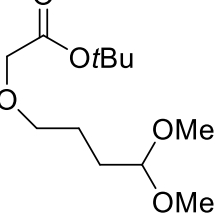

s7

$\left(\mathrm{CDCl}_{3}, 100 \mathrm{MHz}\right)$

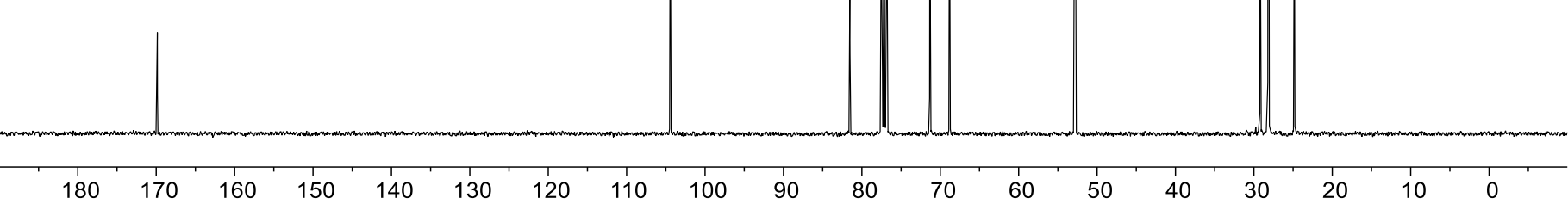




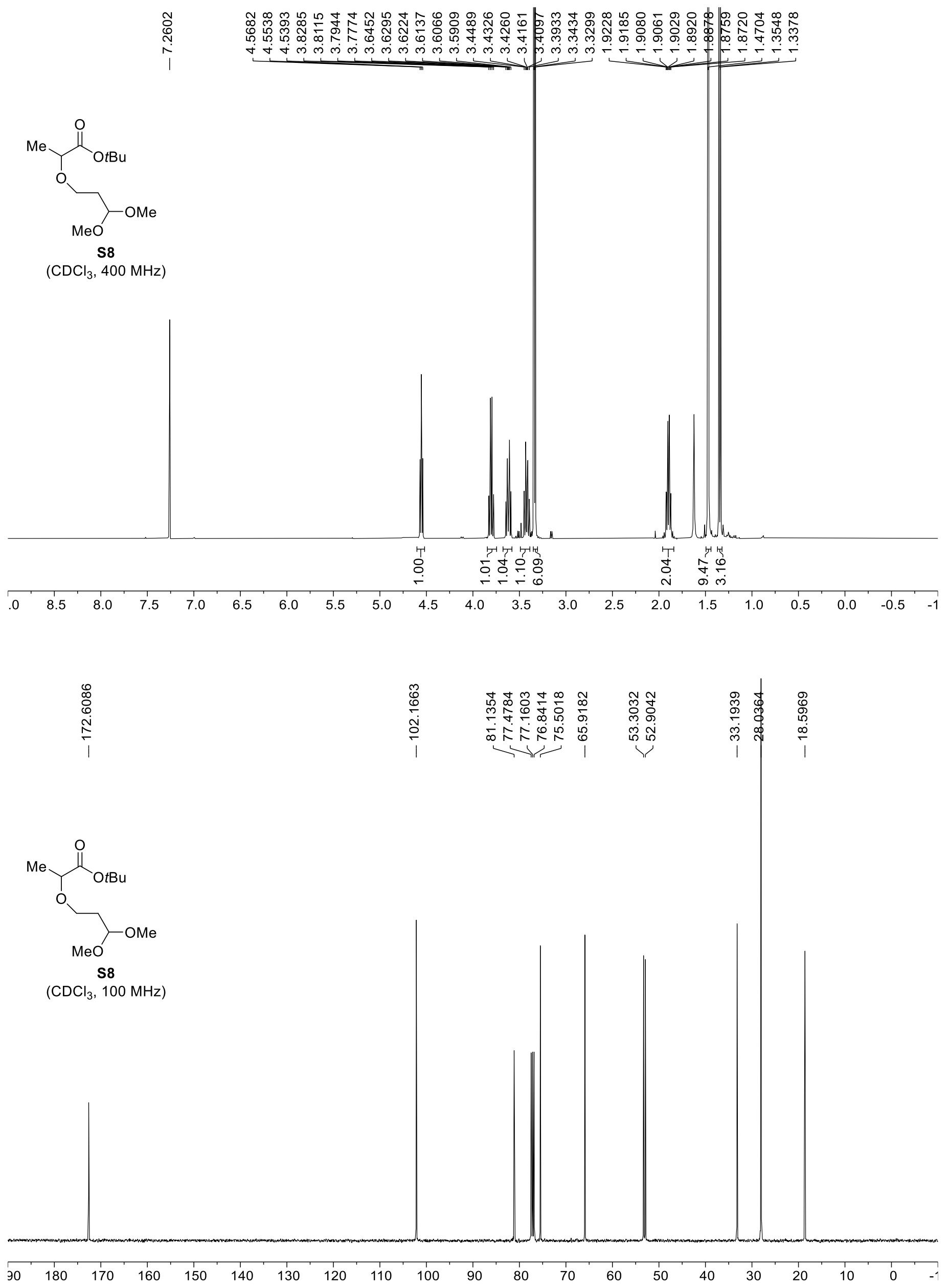




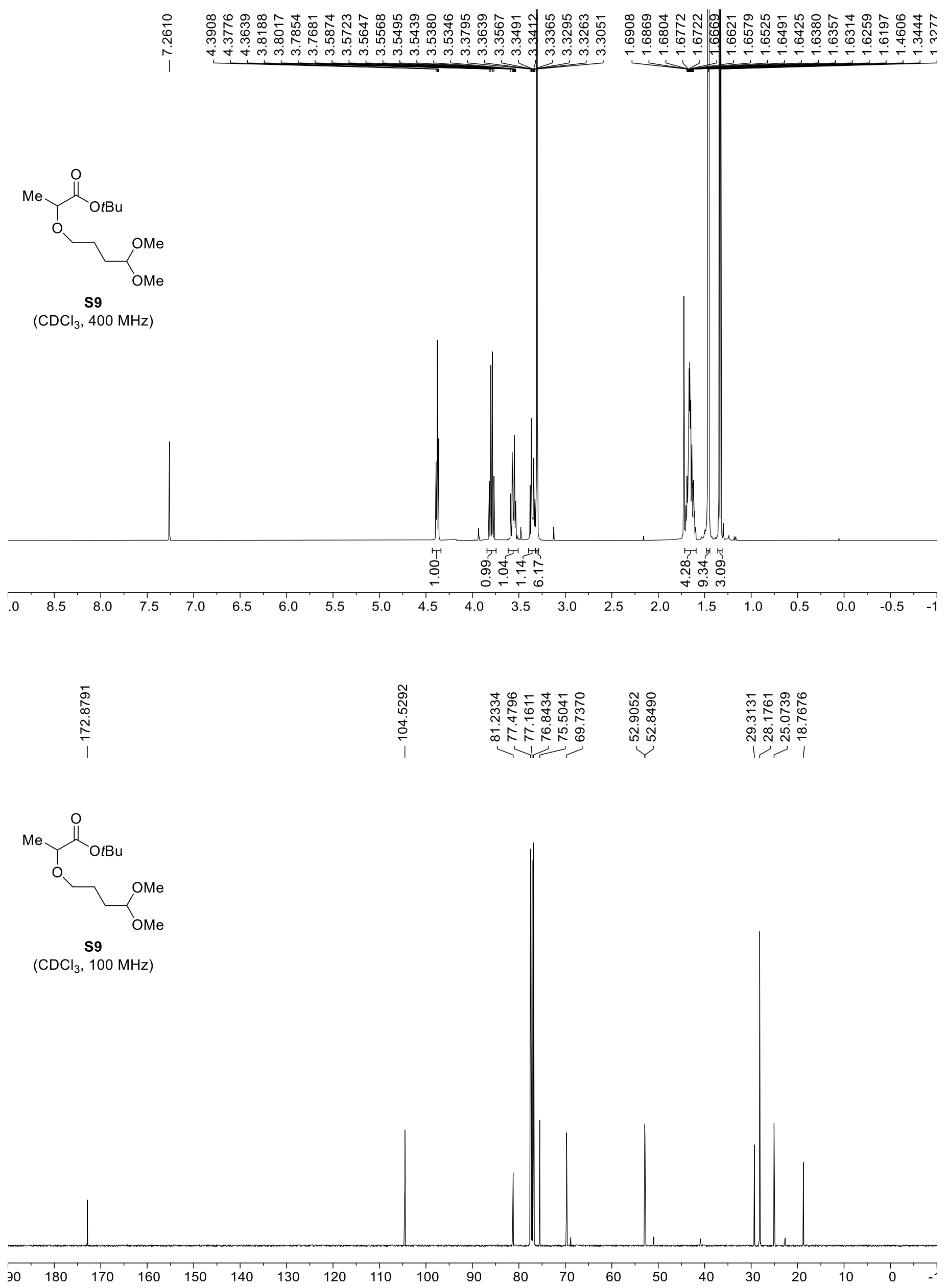


<smiles>CCCOC(=O)C(C)(Cc1cccc(OC)c1)OCCC(OC)OC</smiles>

$\left(\mathrm{CDCl}_{3}, 400 \mathrm{MHz}\right)$

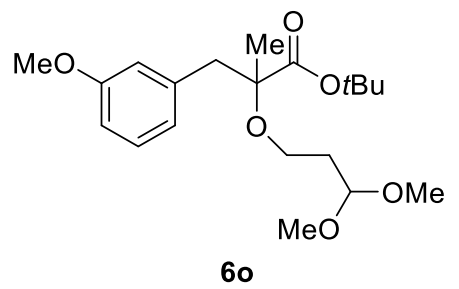

$\left(\mathrm{CDCl}_{3}, 100 \mathrm{MHz}\right)$

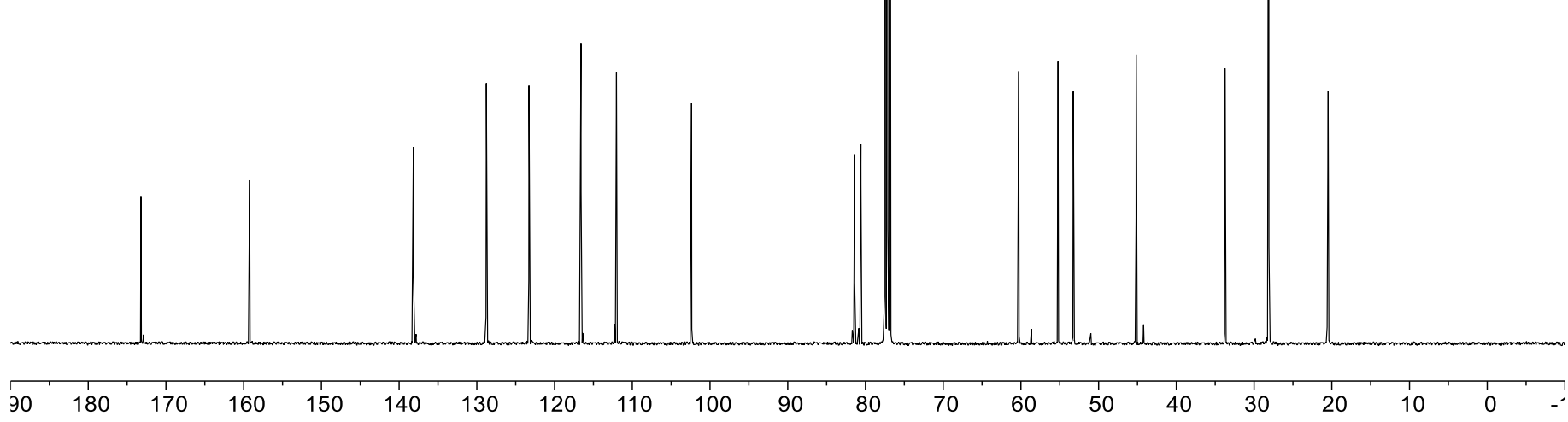




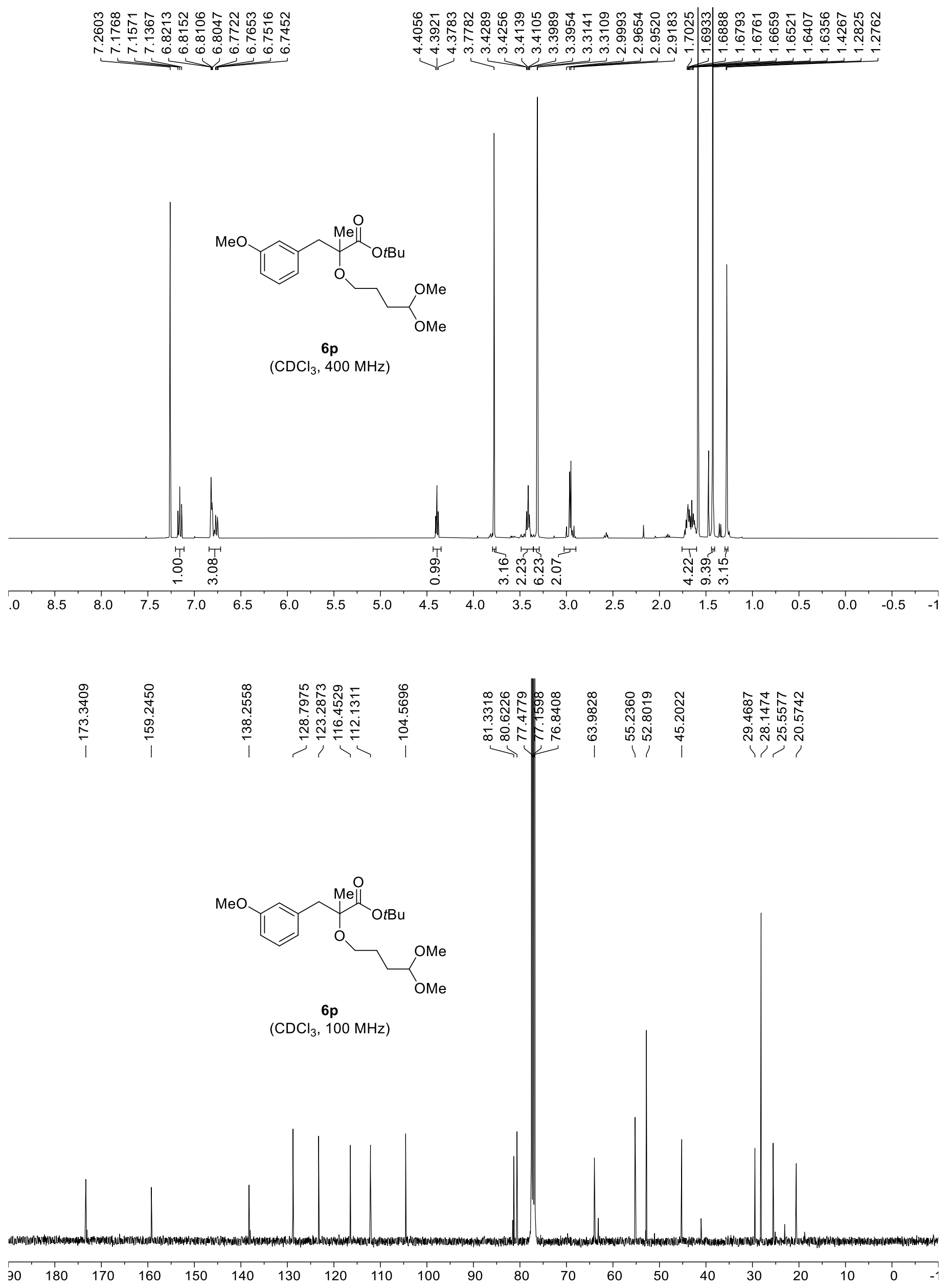




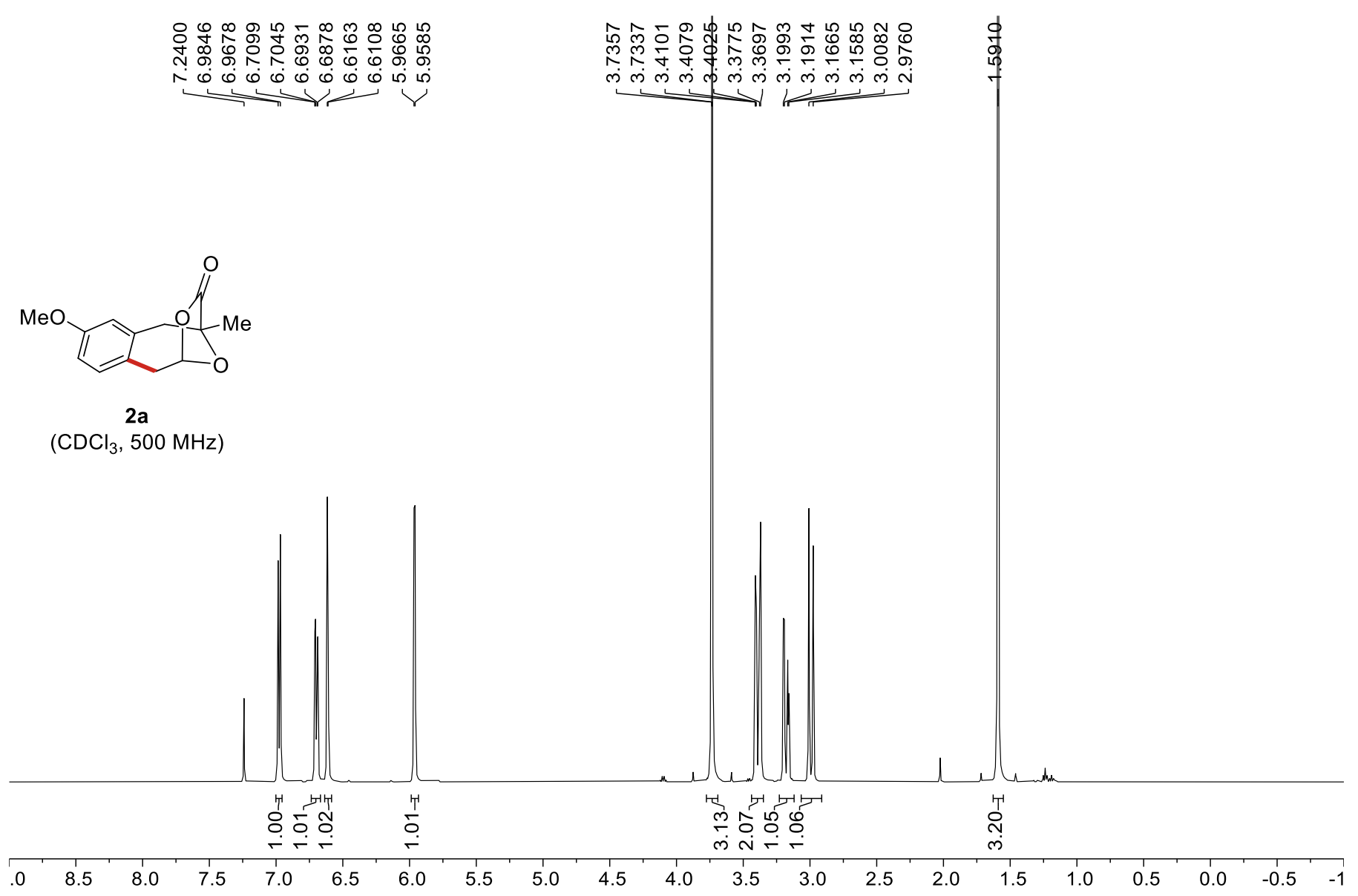

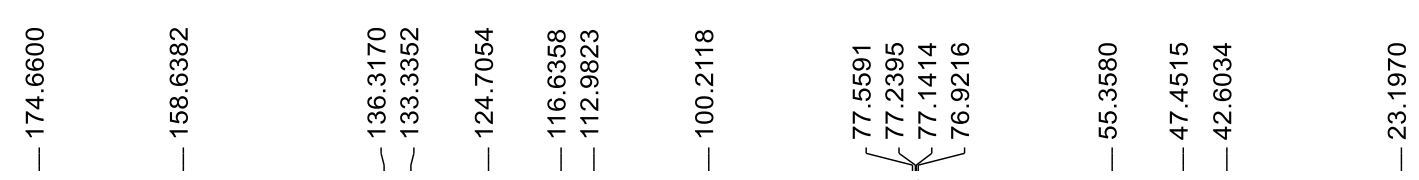

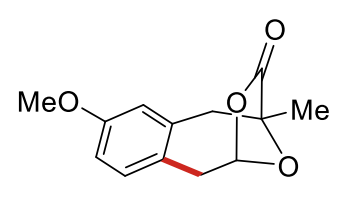

2a

$\left(\mathrm{CDCl}_{3}, 125 \mathrm{MHz}\right)$
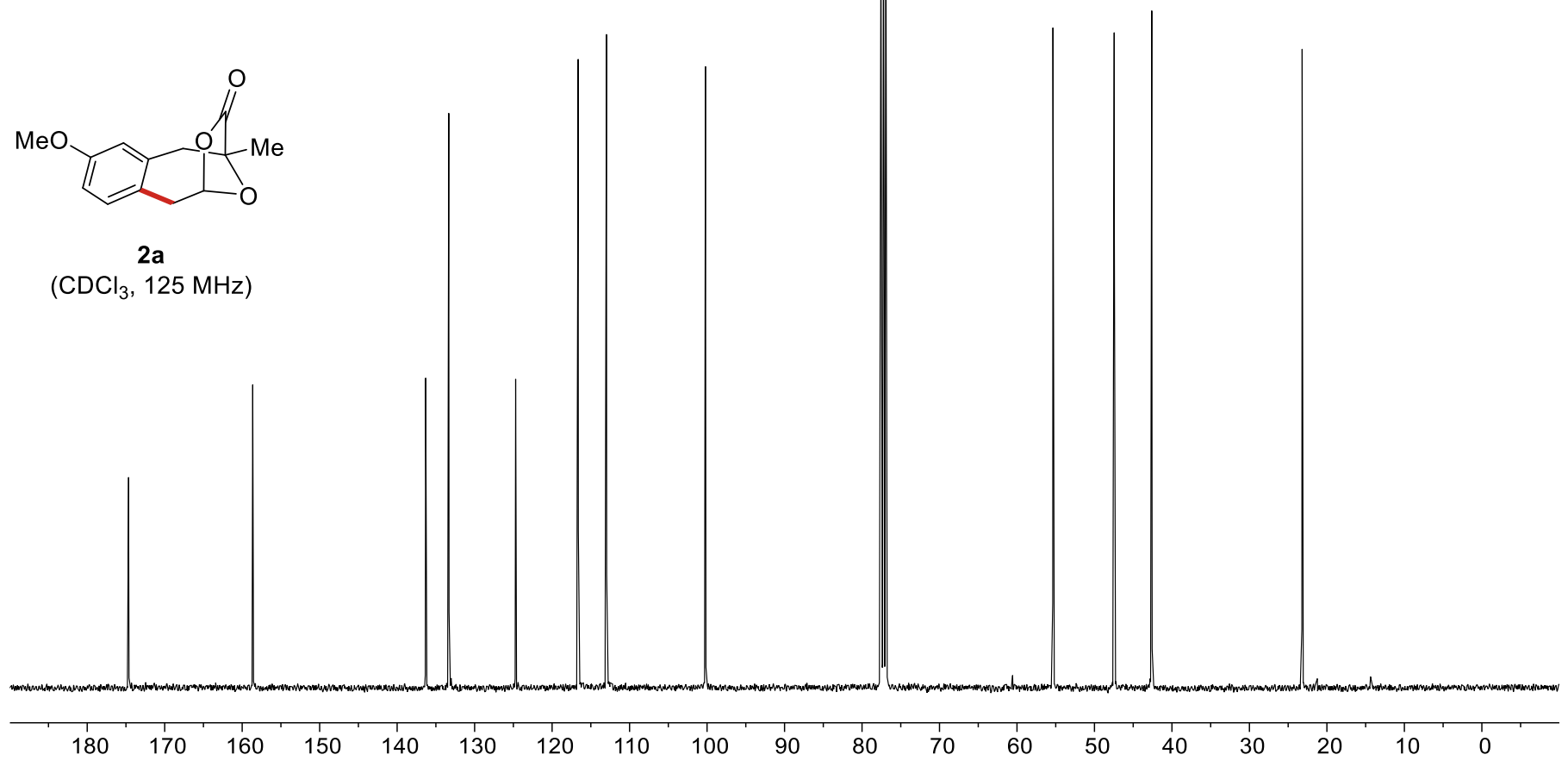


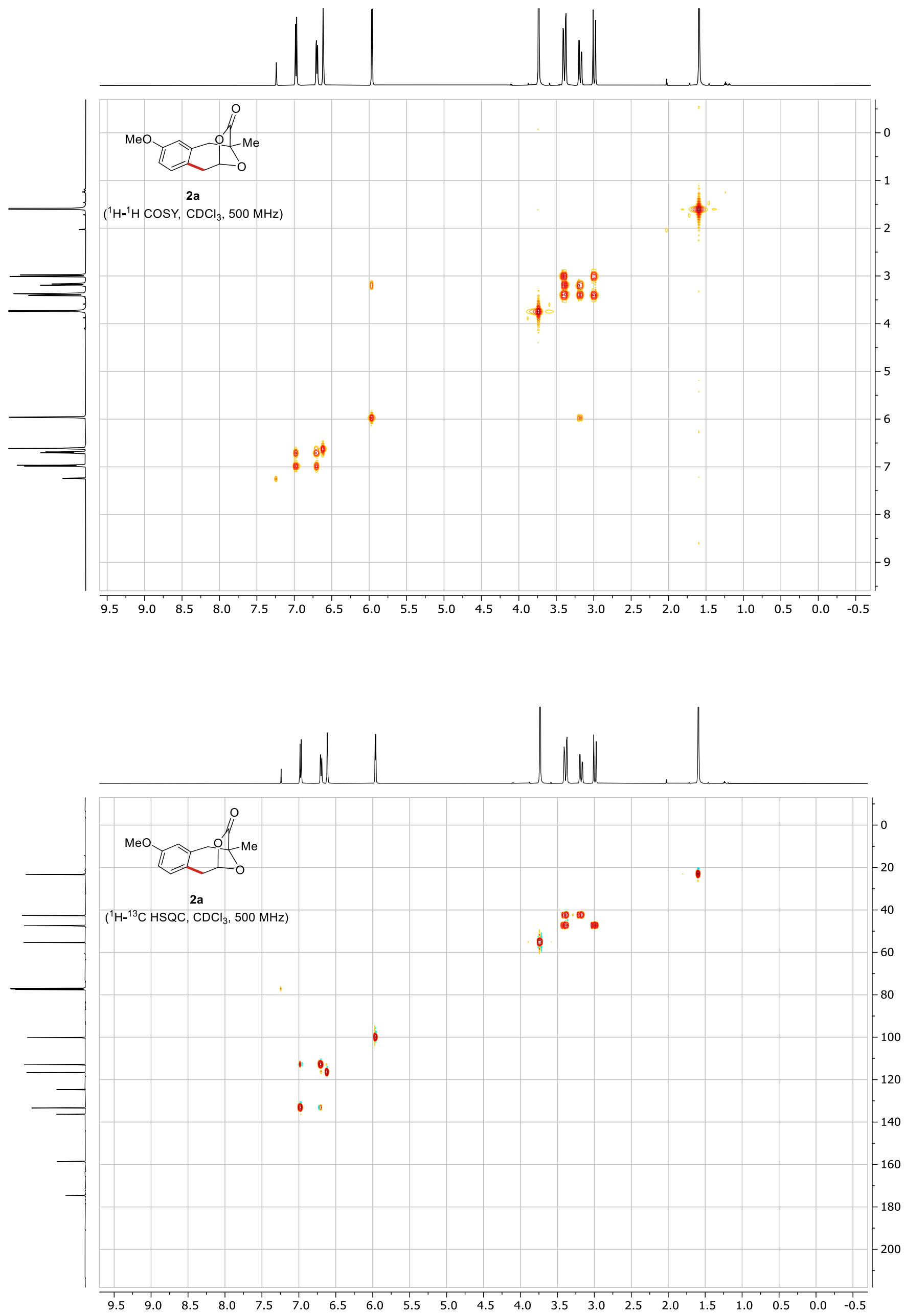




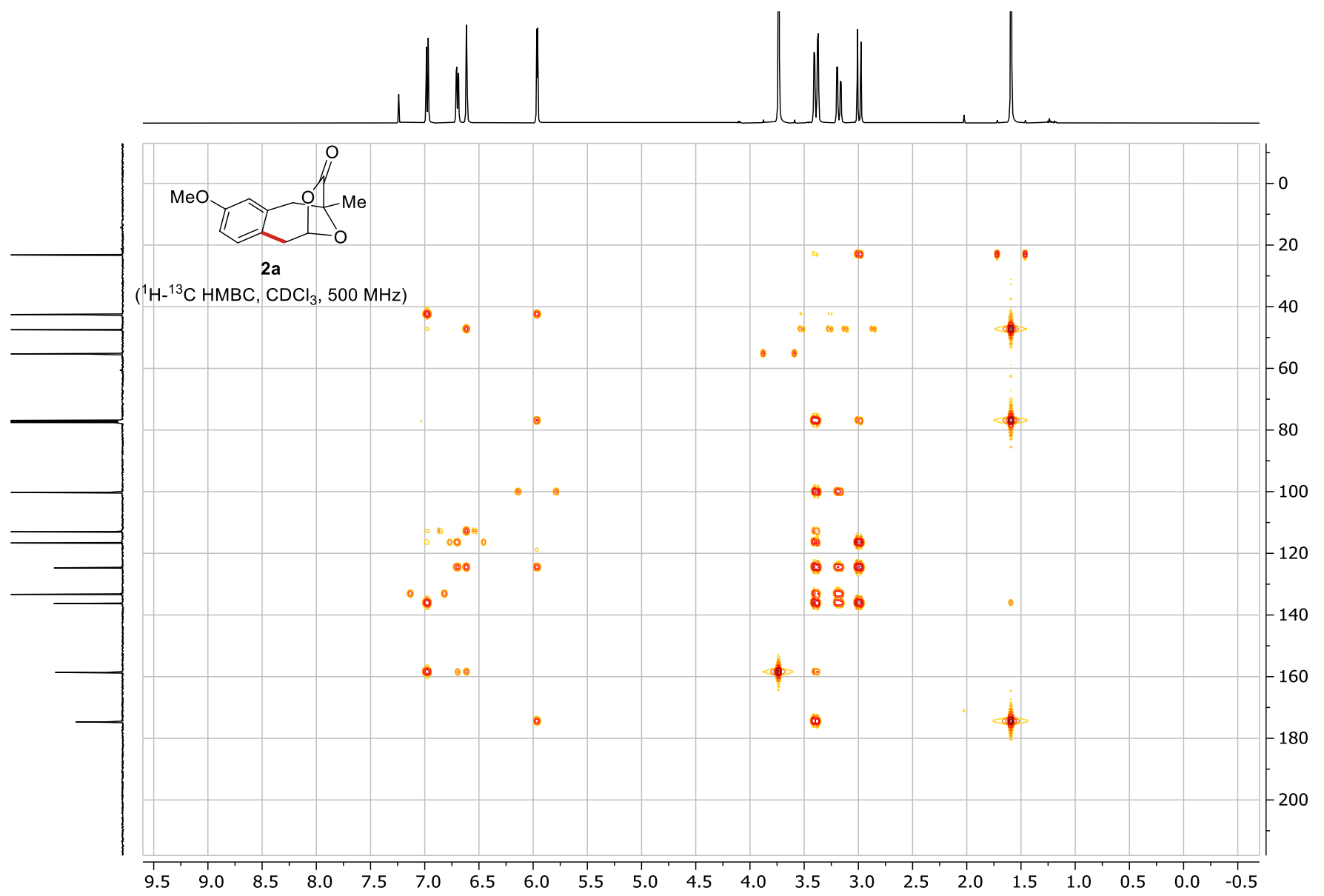

S55 


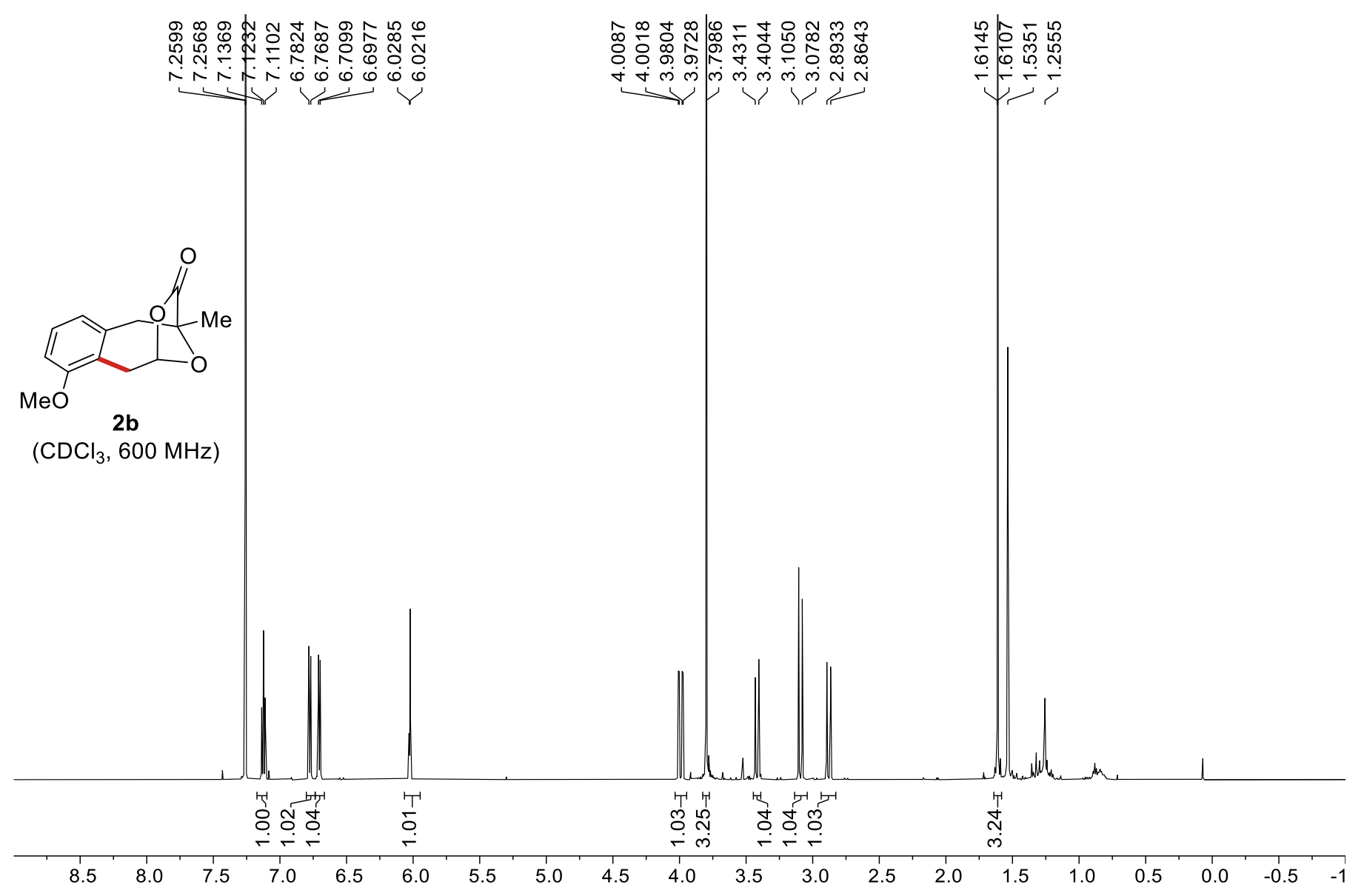

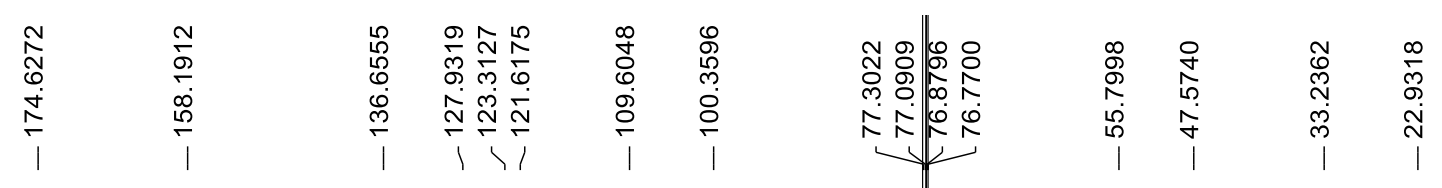

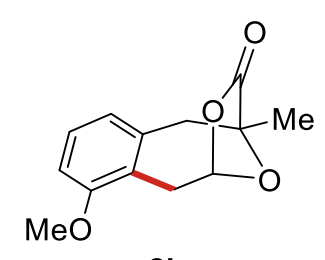

$\left(\mathrm{CDCl}_{3}, 150 \mathrm{MHz}\right)$

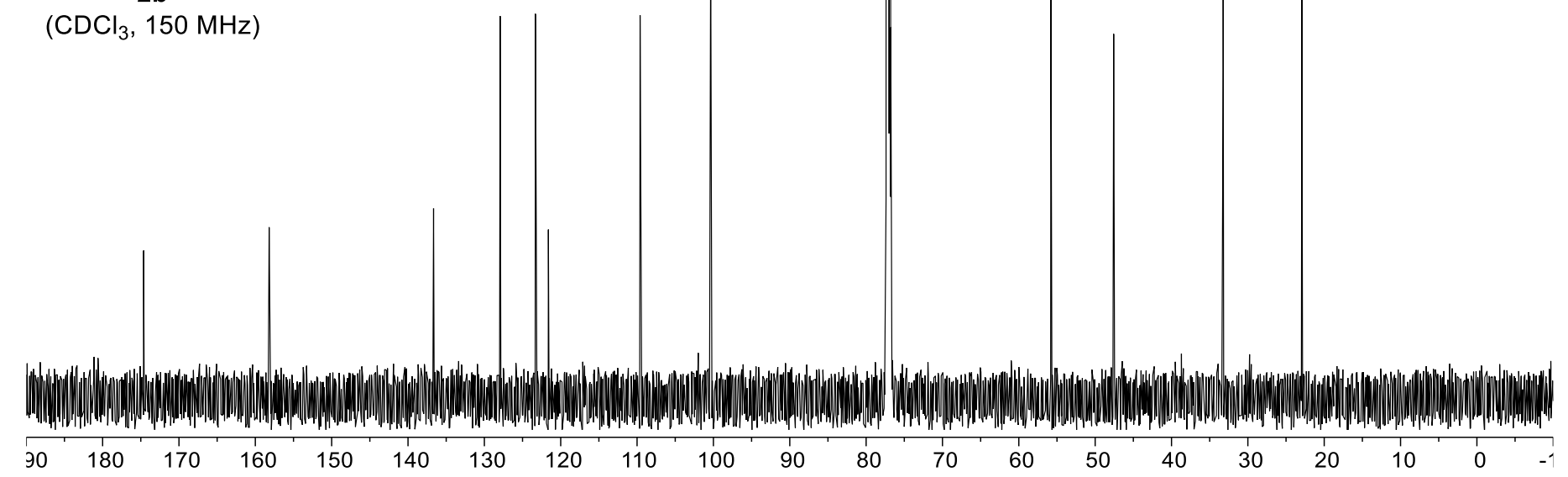




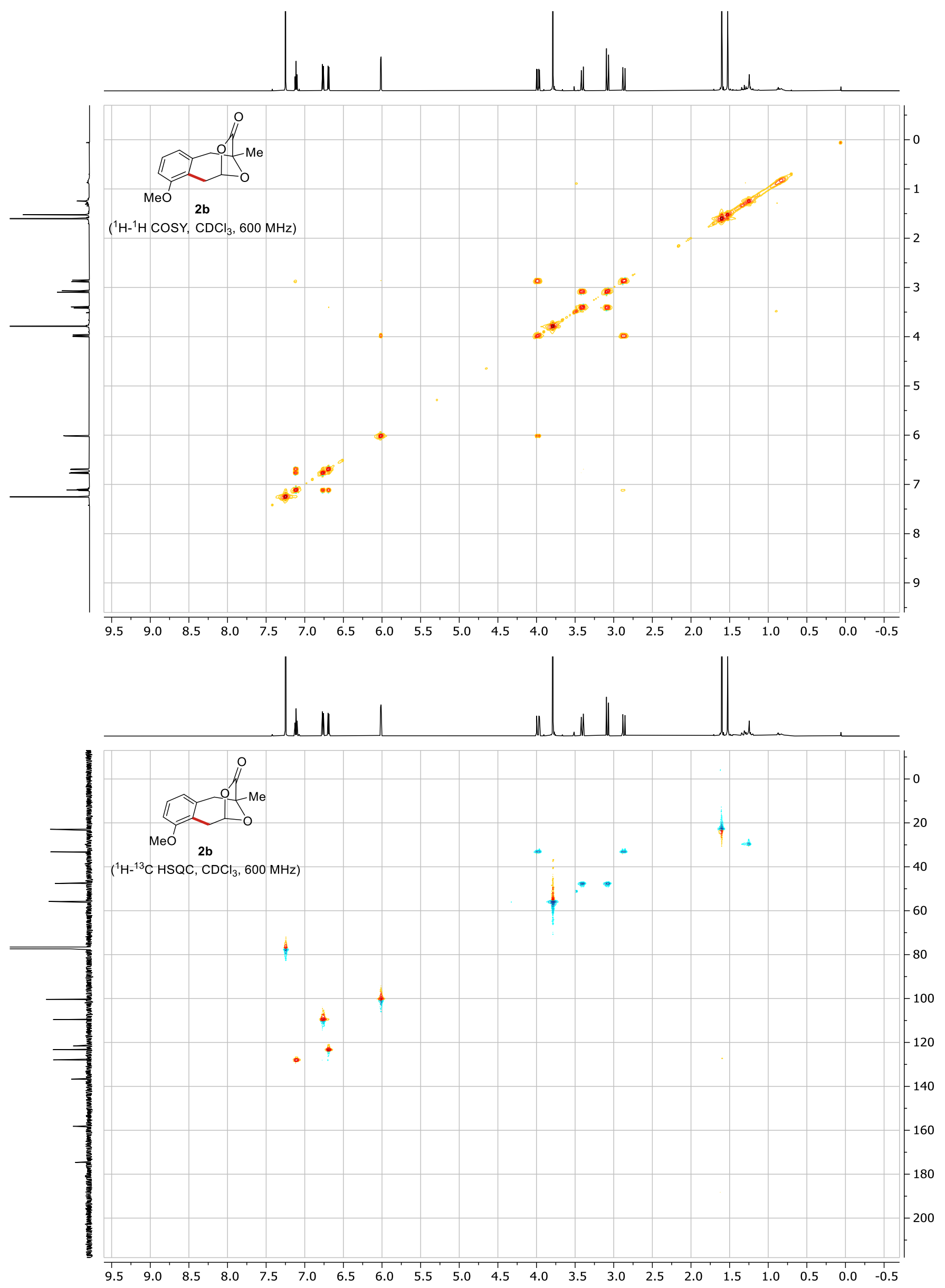

S57 


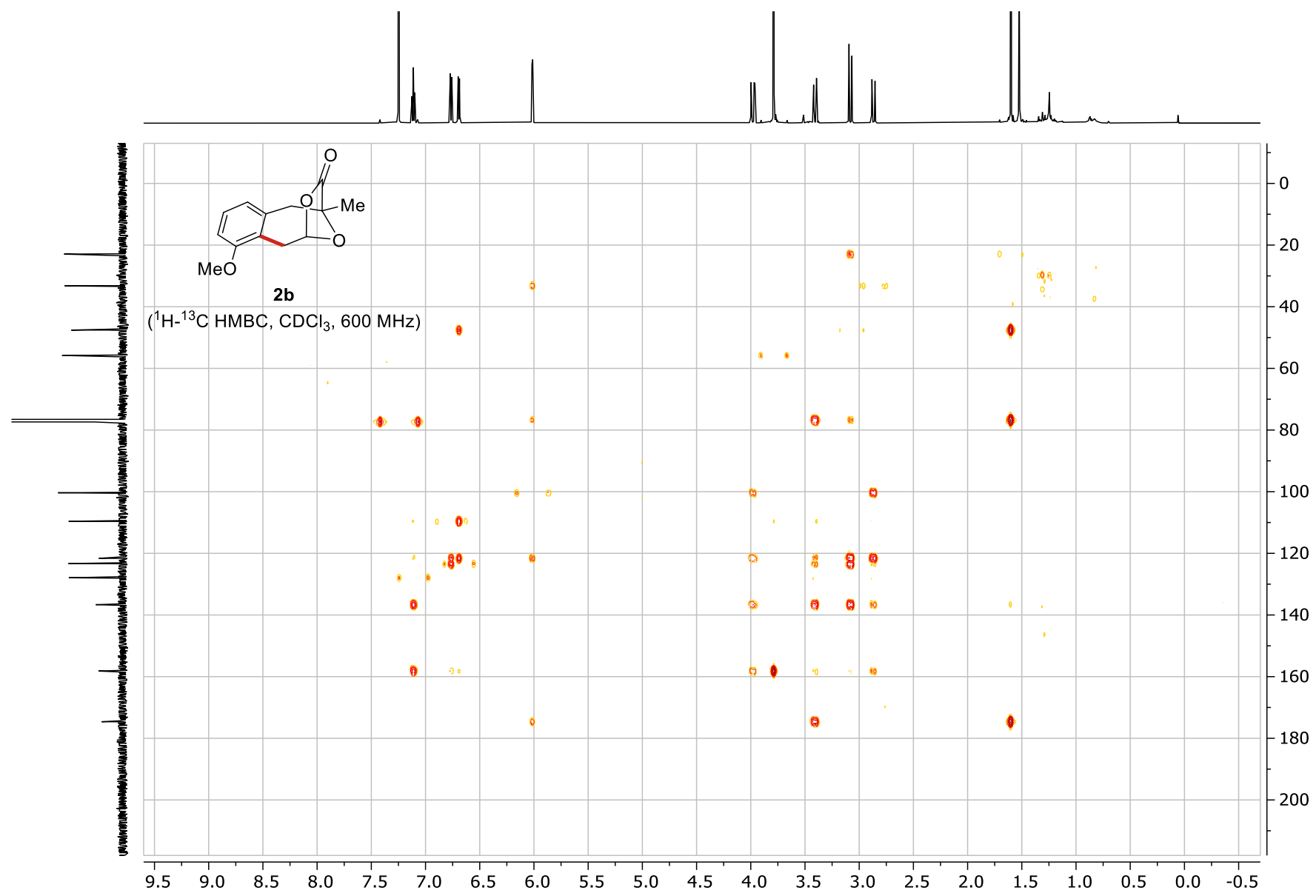

S58 


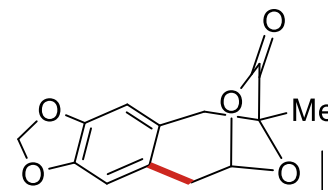

7 a

$\left(\mathrm{CDCl}_{3}, 400 \mathrm{MHz}\right)$

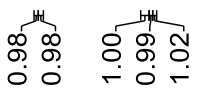

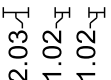

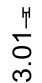

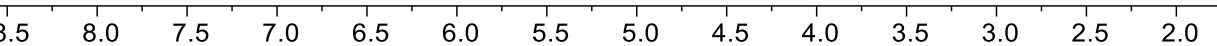

\begin{tabular}{|c|c|c|c|c|c|c|}
\hline $\begin{array}{l}\infty \\
\infty \\
\vdots \\
0 \\
+ \\
+ \\
亡 \\
\end{array}$ & 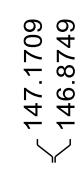 & 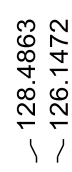 & 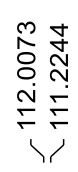 & 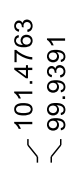 & 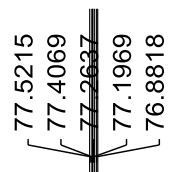 & 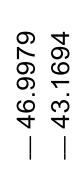 \\
\hline
\end{tabular}

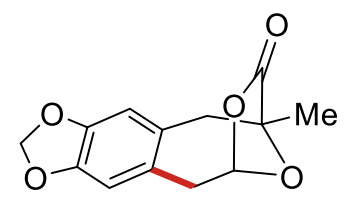

$7 a$

$\left(\mathrm{CDCl}_{3}, 100 \mathrm{MHz}\right)$

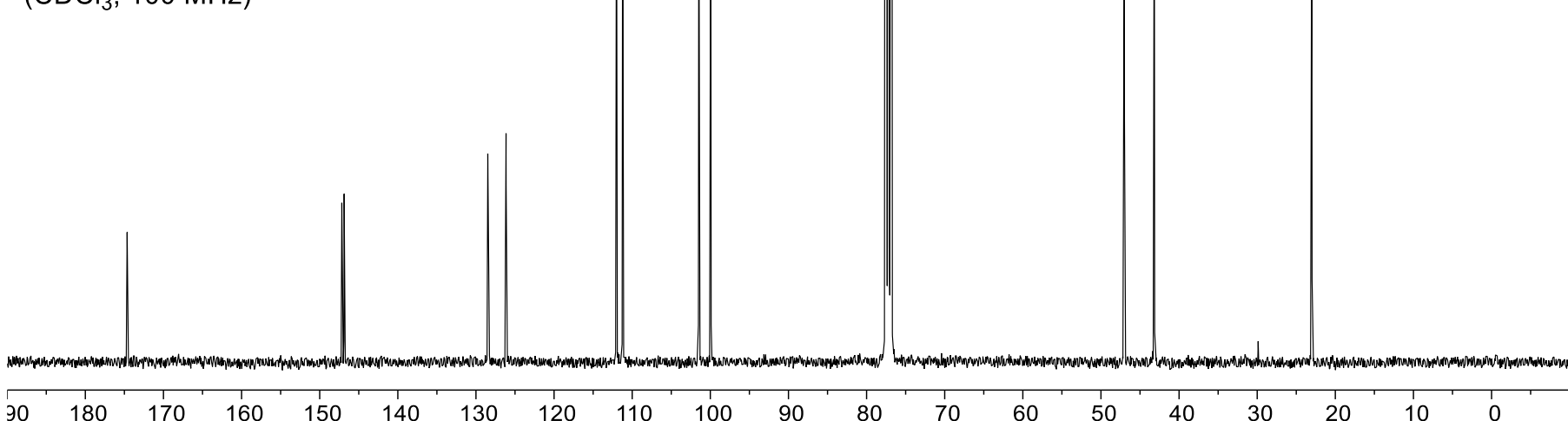




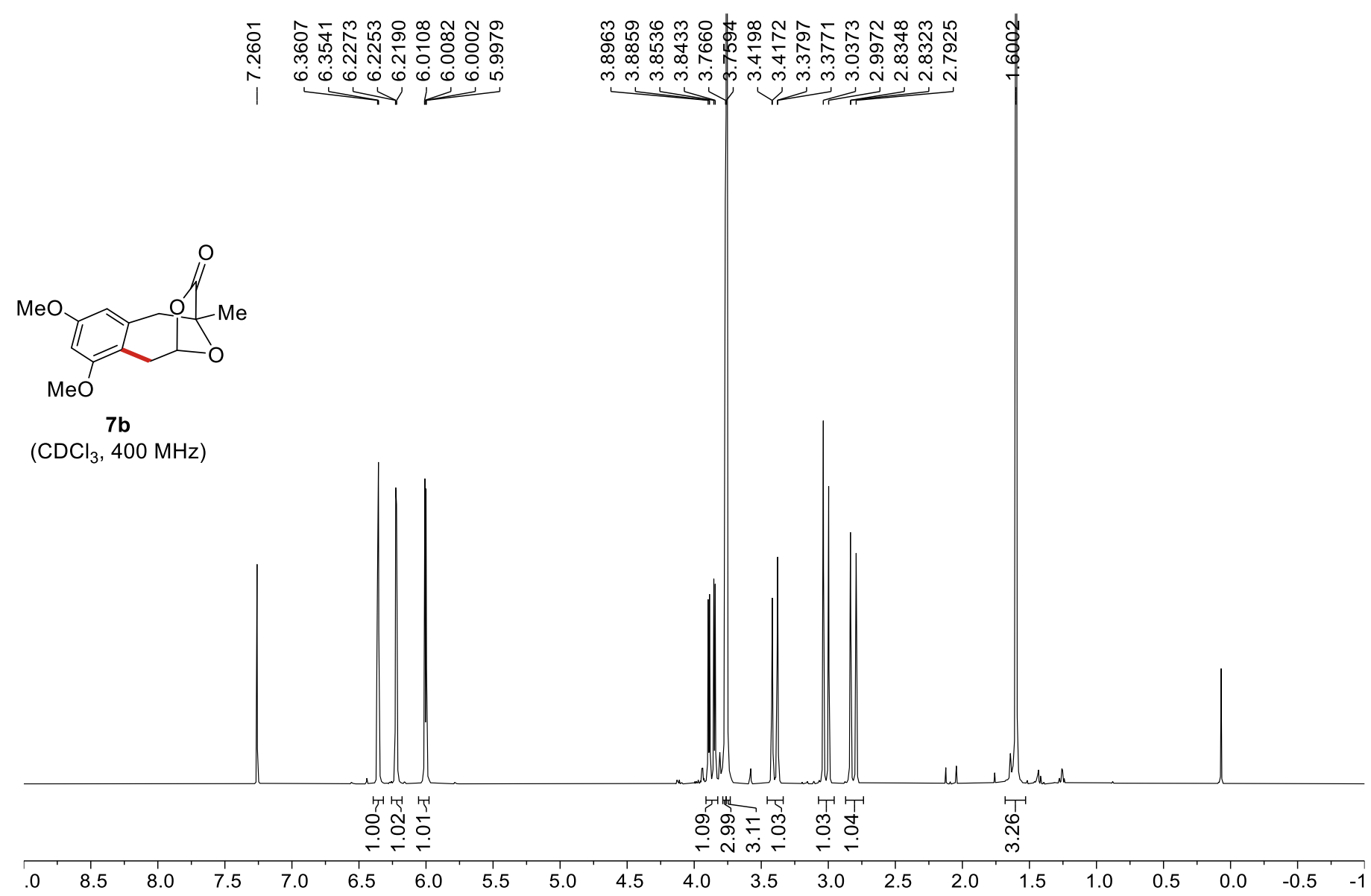

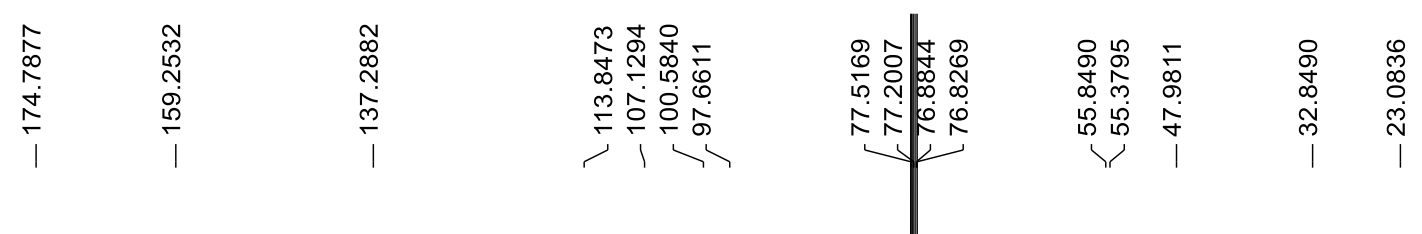

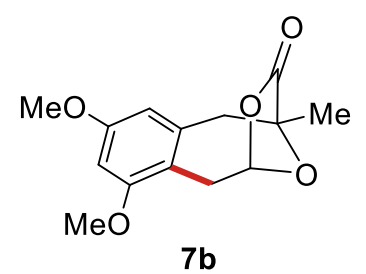

$\left(\mathrm{CDCl}_{3}, 100 \mathrm{MHz}\right)$

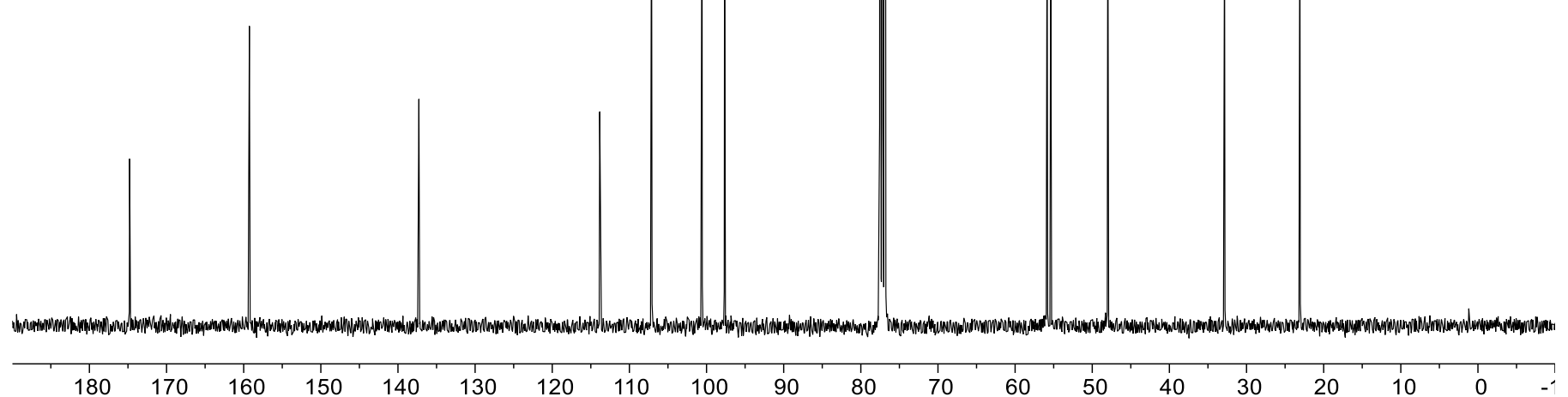



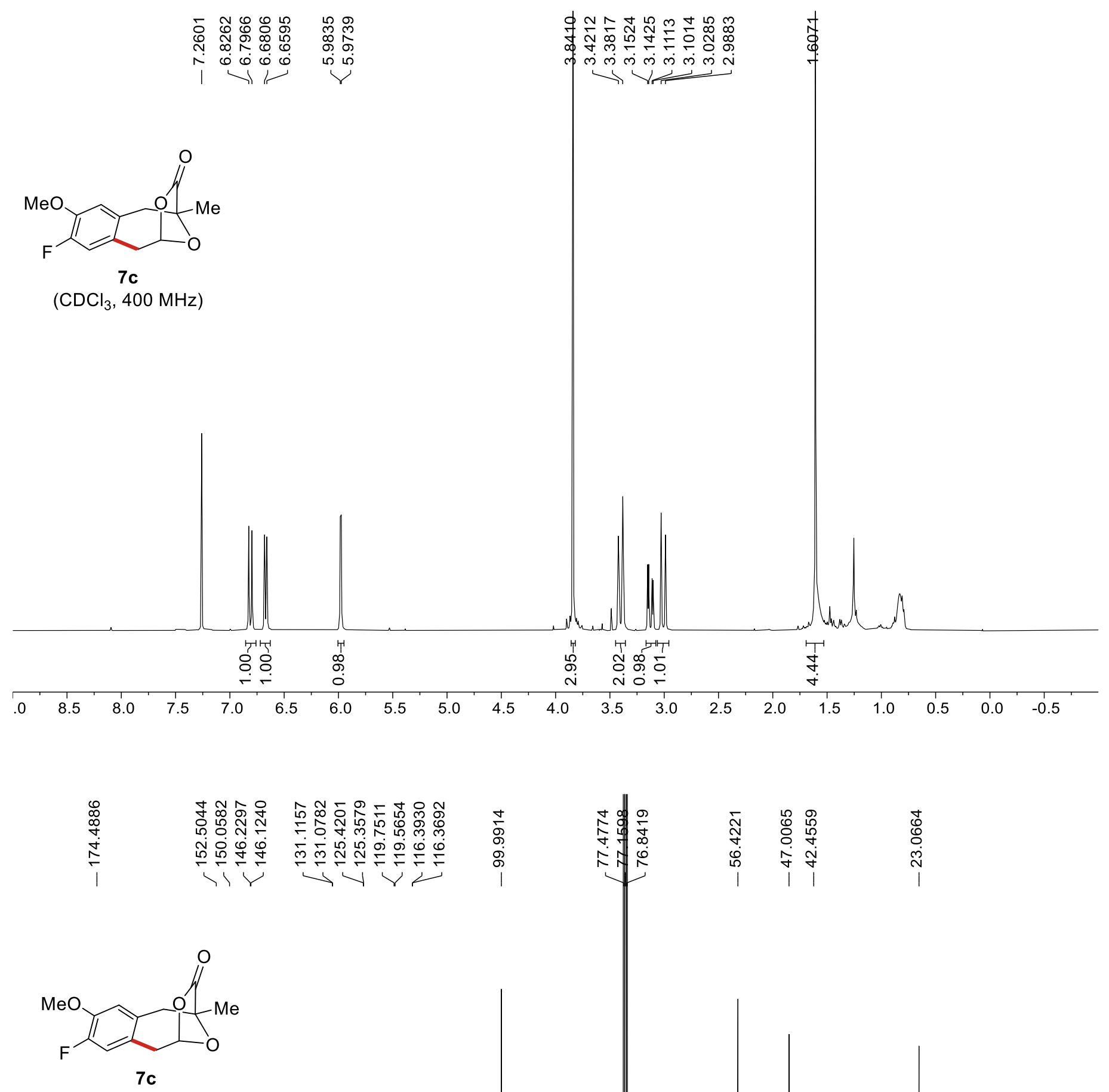

$\left(\mathrm{CDCl}_{3}, 100 \mathrm{MHz}\right)$

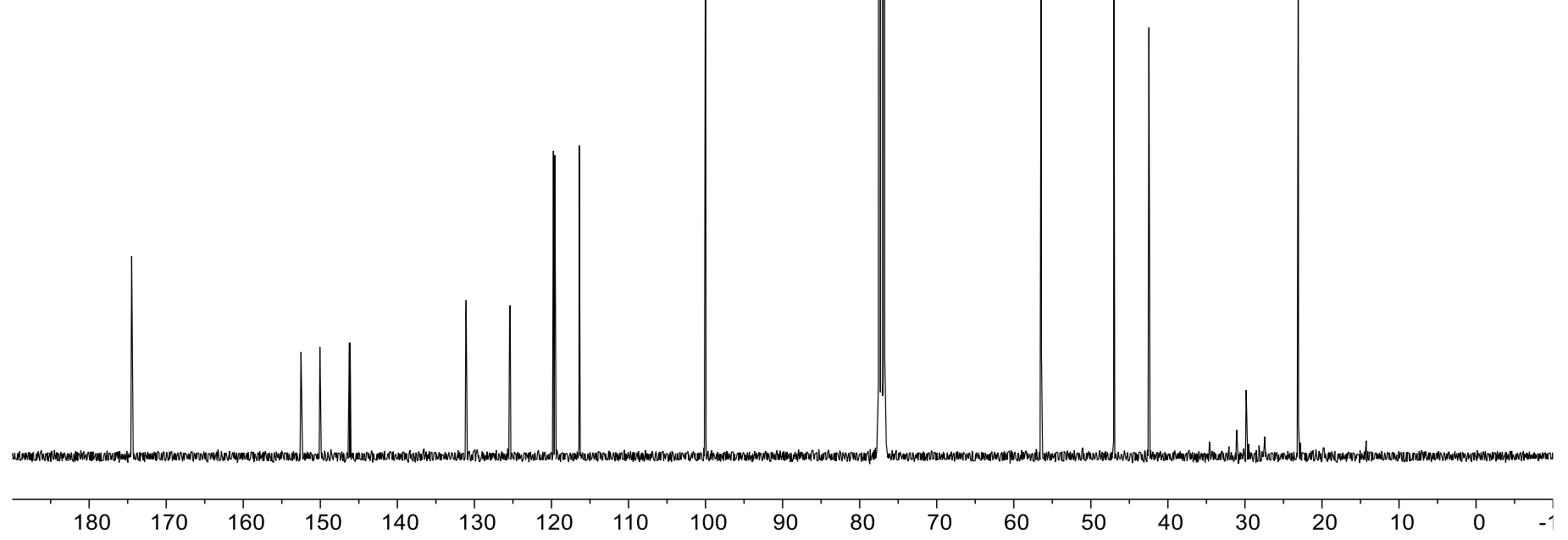




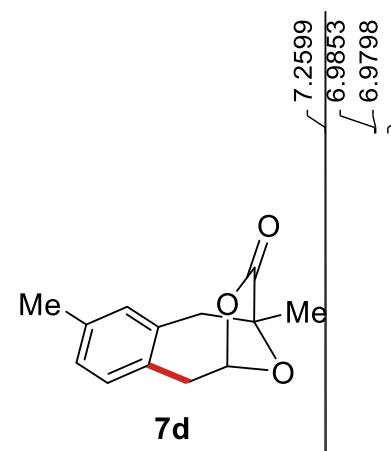

$\left(\mathrm{CDCl}_{3}, 400 \mathrm{MHz}\right)$

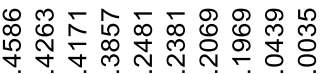

ले लेल लं लं लं ले

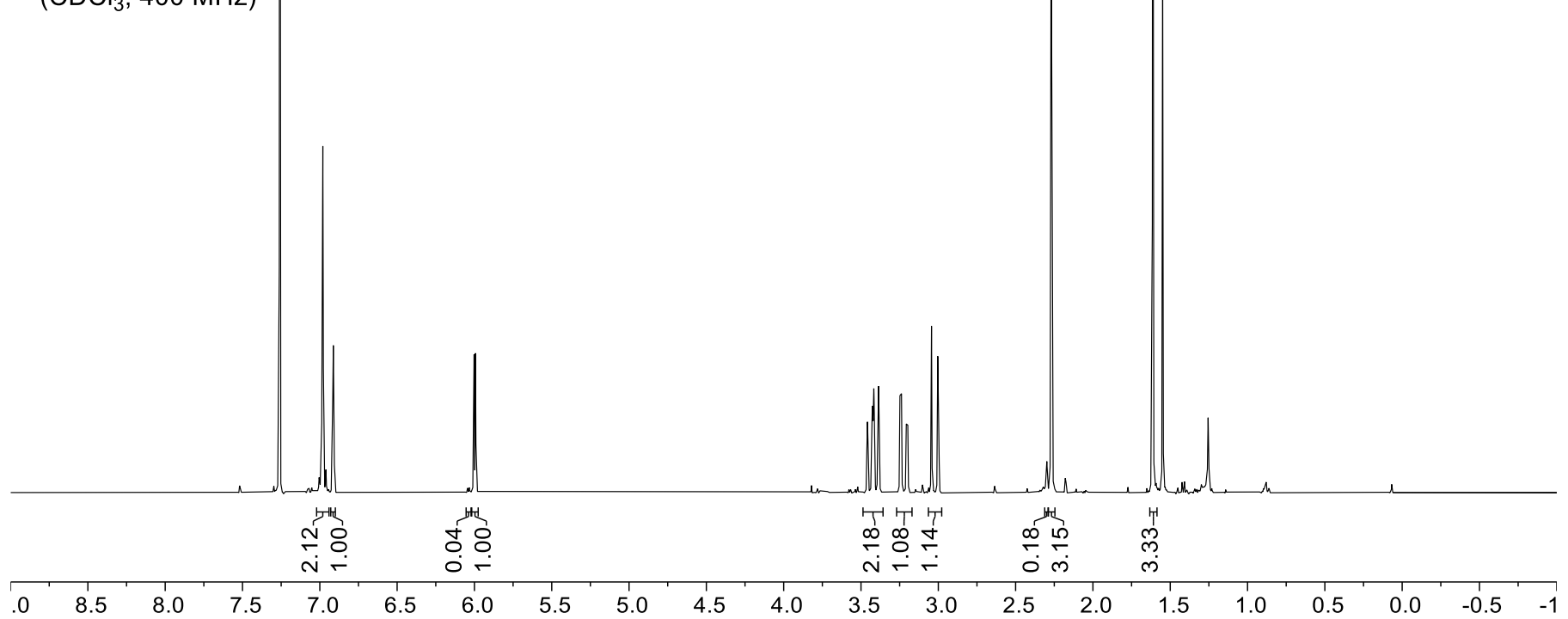<smiles>Cc1ccc2c(c1)OC1(C)OC(=O)C2O1</smiles>

$7 d$

$\left(\mathrm{CDCl}_{3}, 200 \mathrm{MHz}\right)$

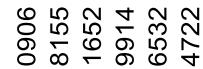

虫

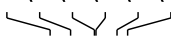

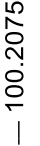

ల్ల

N N

s
ิㅗำ

लें

।

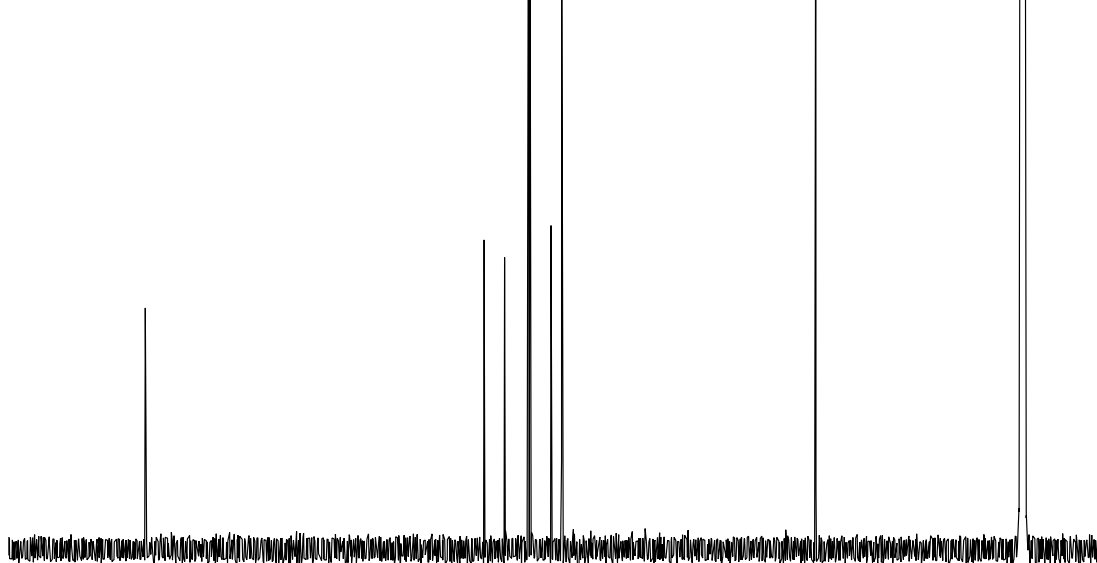




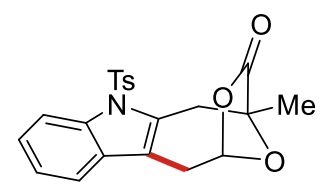

$7 \mathrm{~h}$

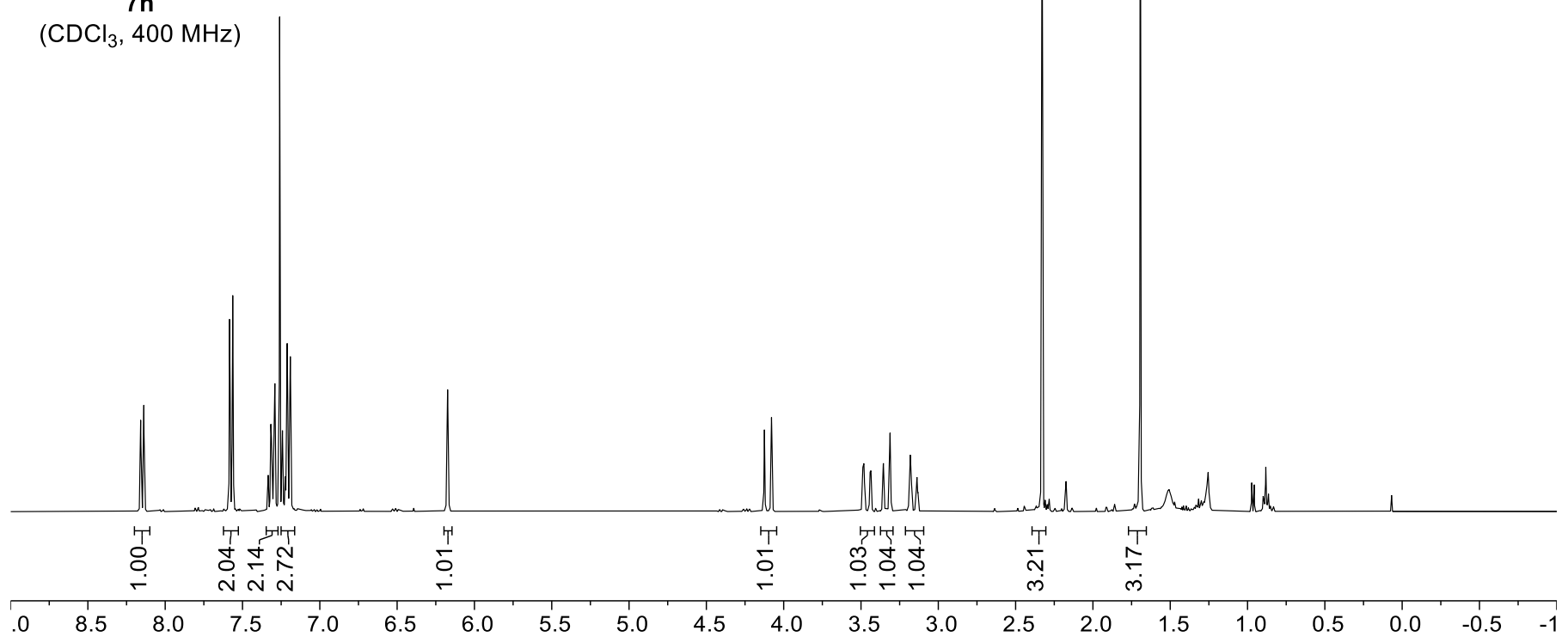

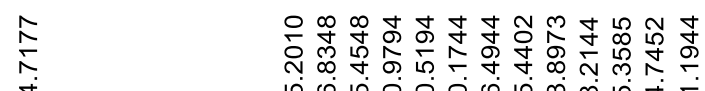

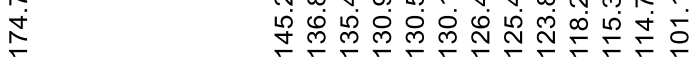

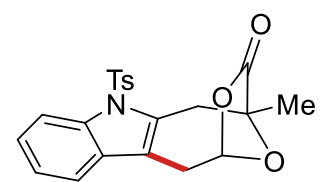

|

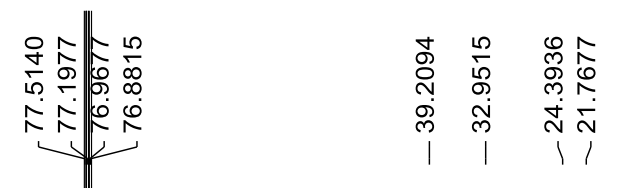

$7 \mathrm{~h}$

$\left(\mathrm{CDCl}_{3}, 100 \mathrm{MHz}\right)$

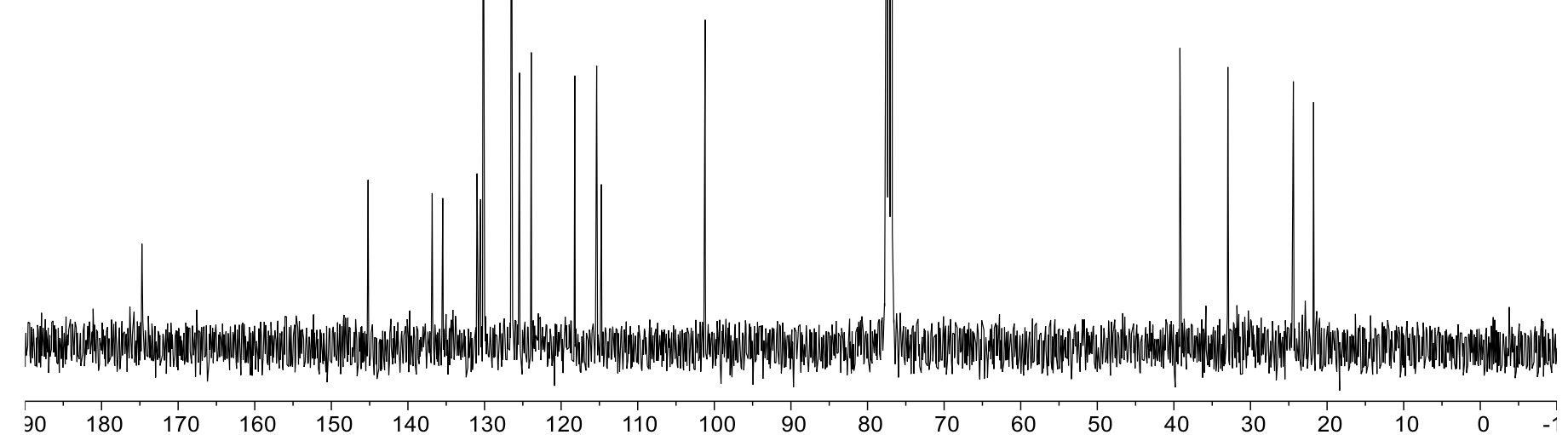




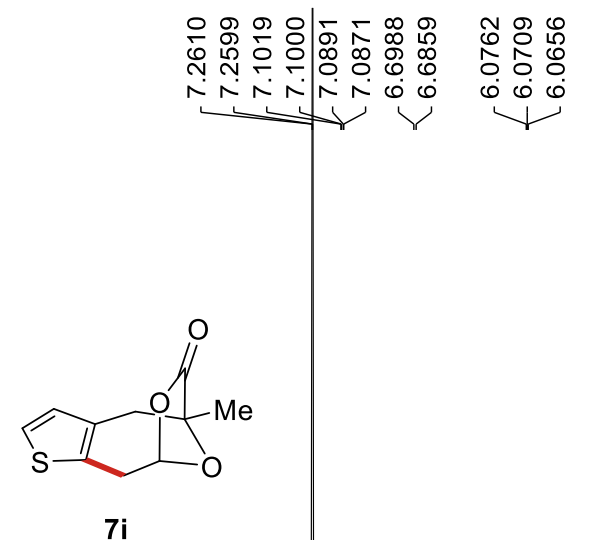

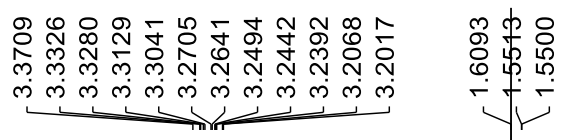

$\left(\mathrm{CDCl}_{3}, 400 \mathrm{MHz}\right)$
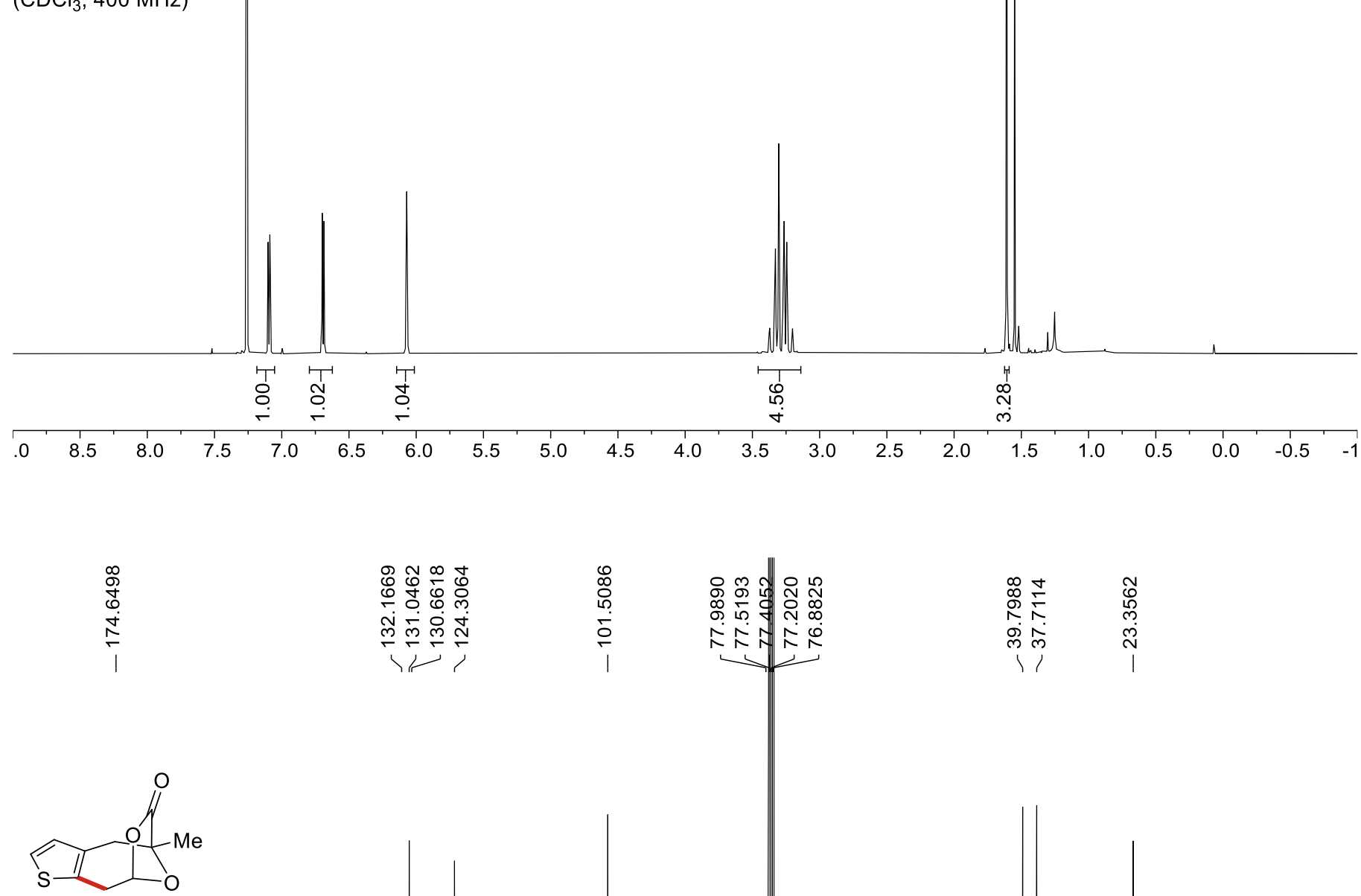

$7 i$

$\left(\mathrm{CDCl}_{3}, 100 \mathrm{MHz}\right)$

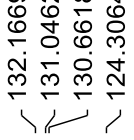
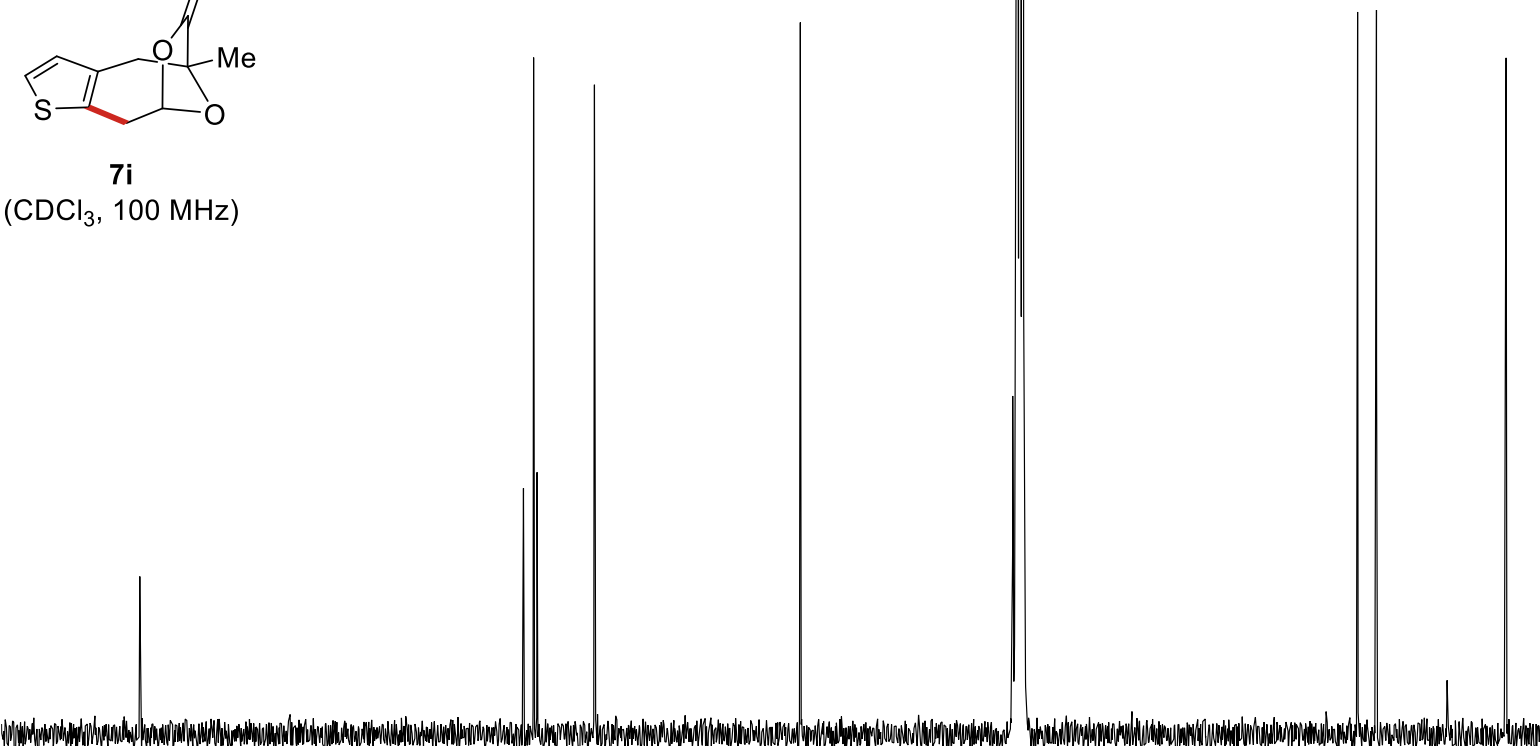

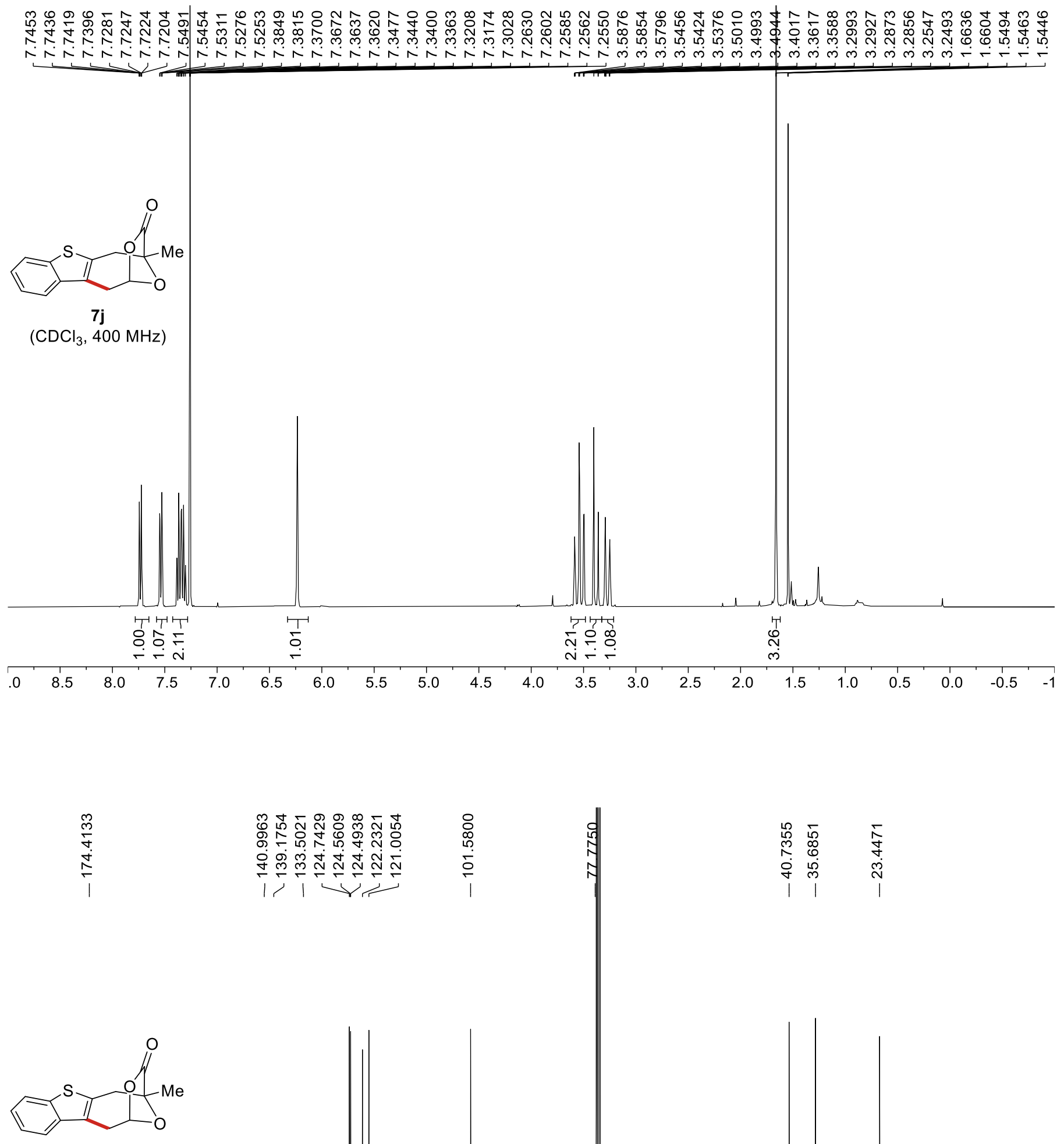

$7 \mathbf{j}$

$\left(\mathrm{CDCl}_{3}, 100 \mathrm{MHz}\right)$

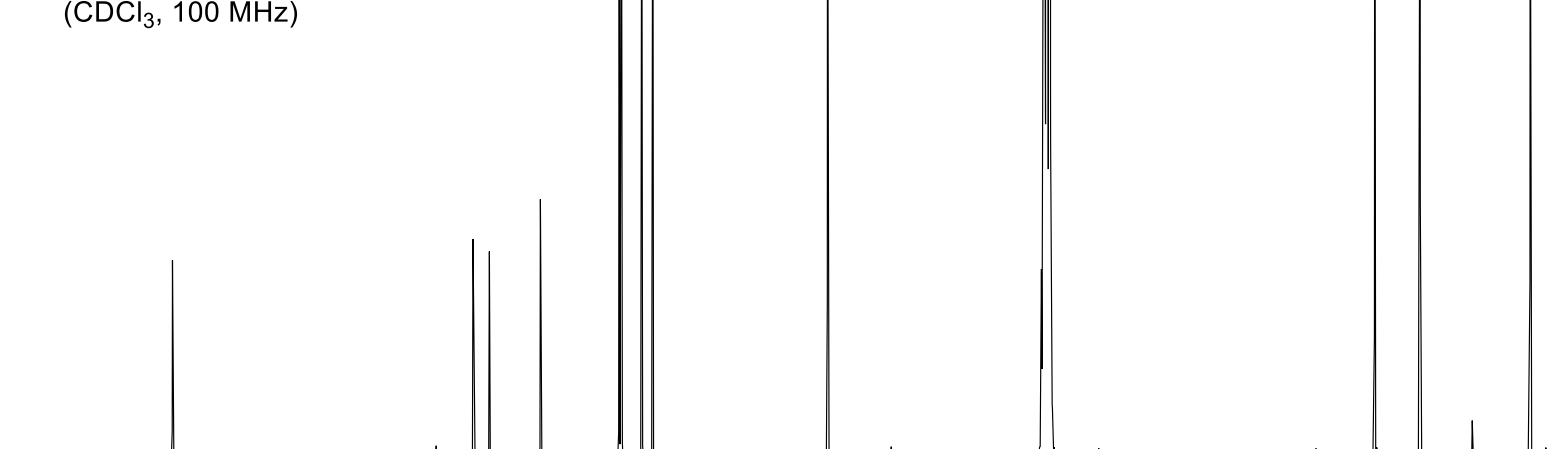




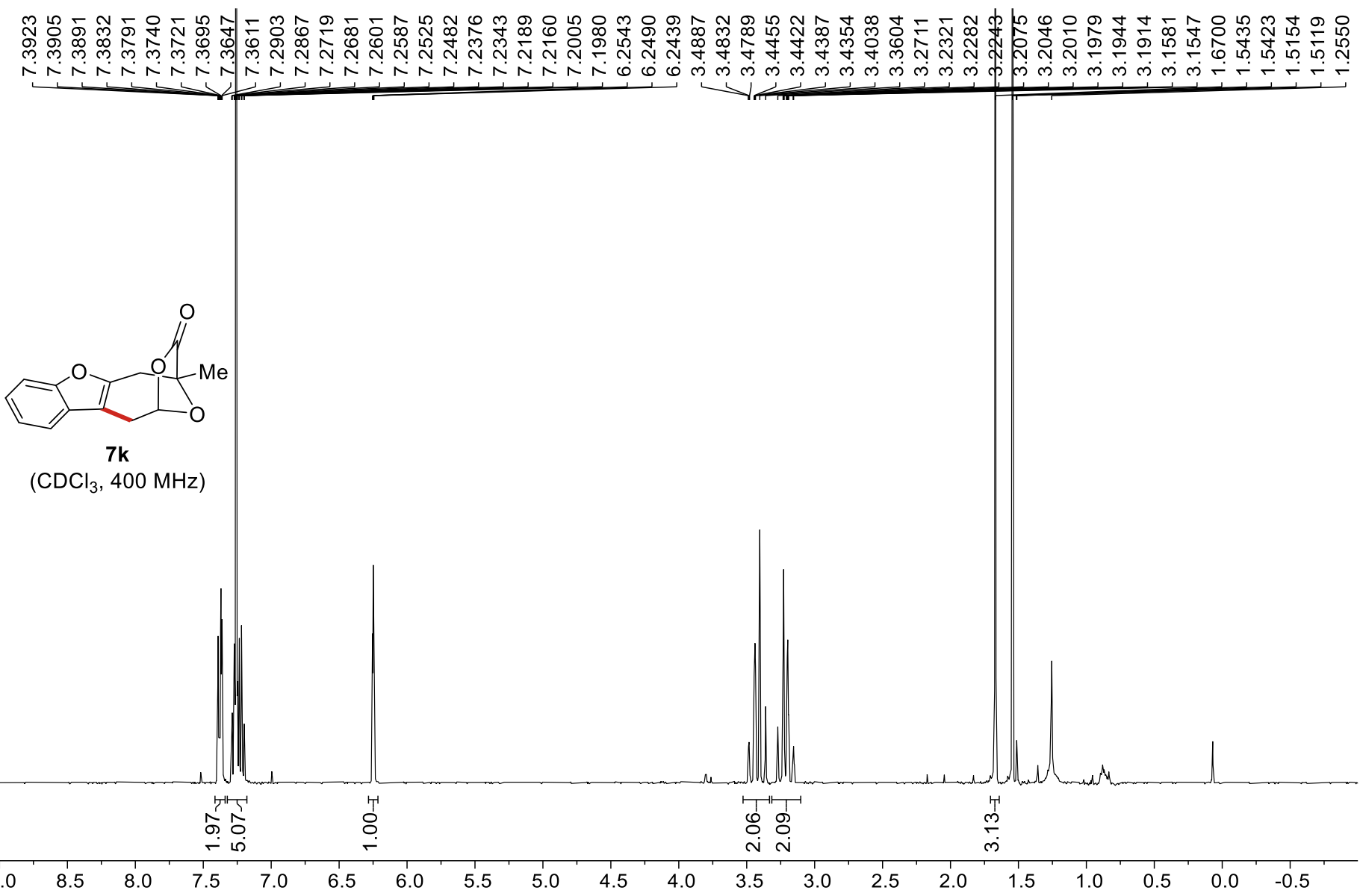

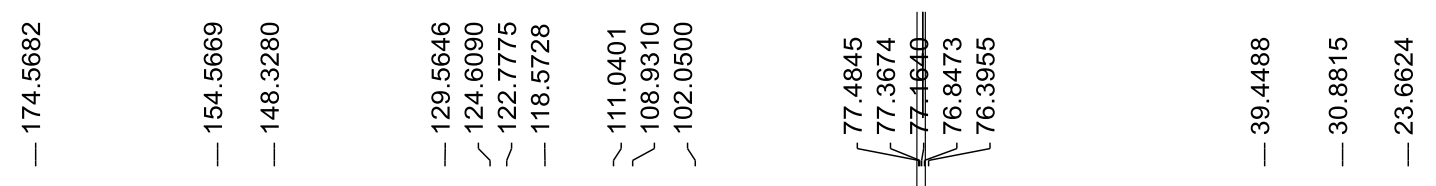

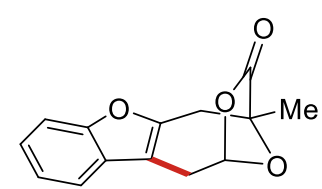

$7 k$

$\left(\mathrm{CDCl}_{3}, 100 \mathrm{MHz}\right)$

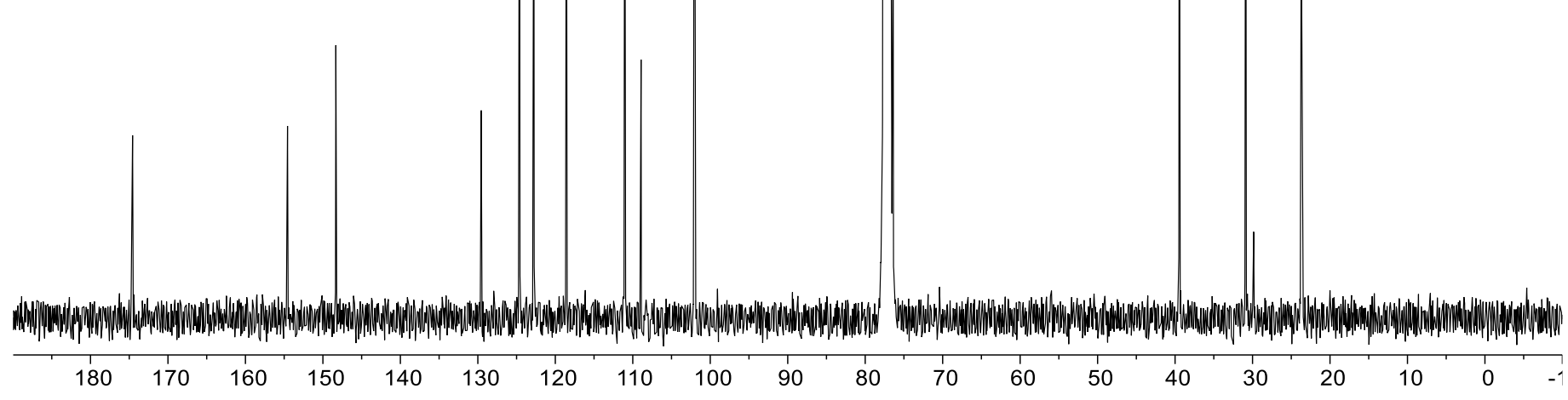



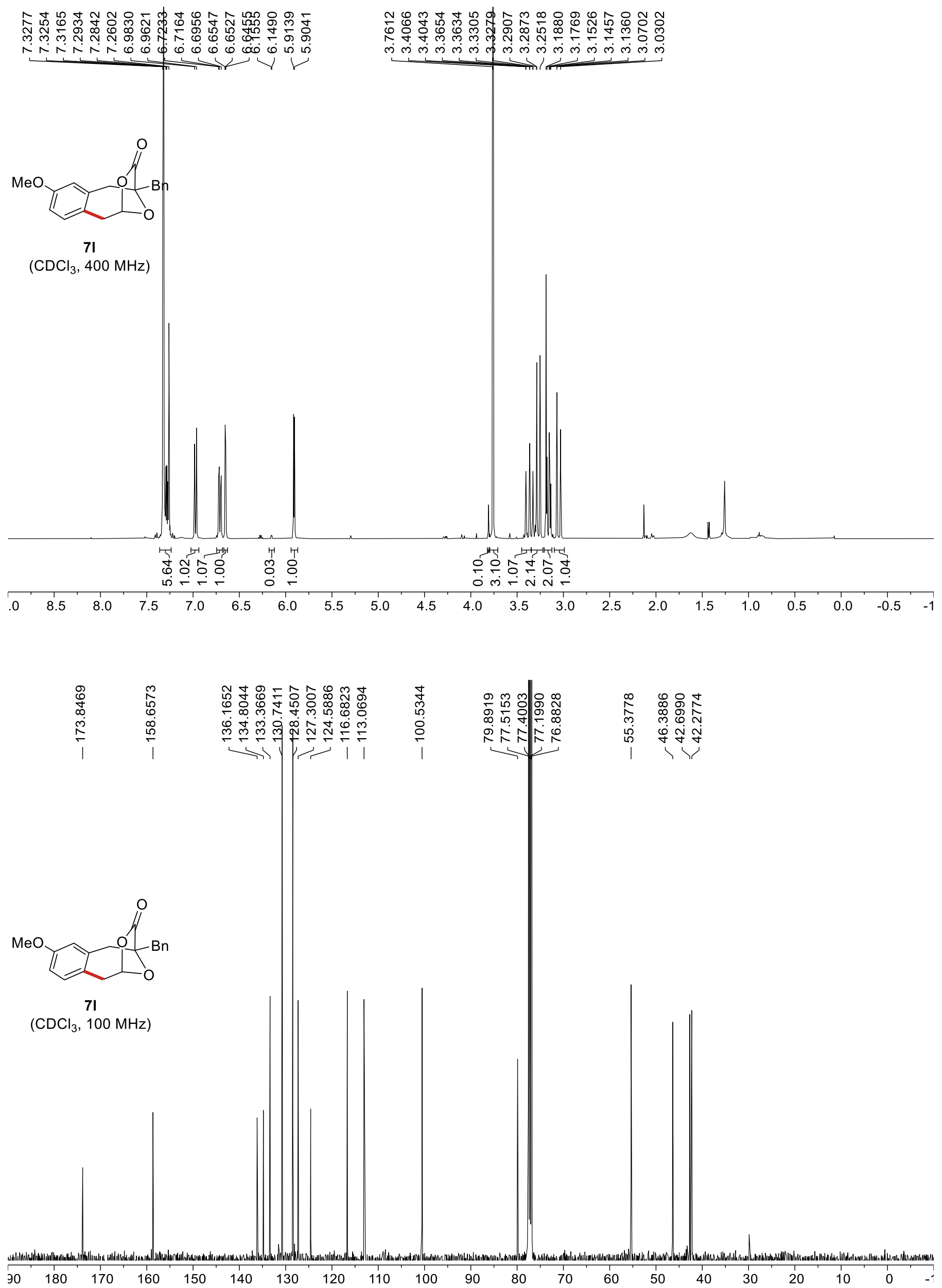


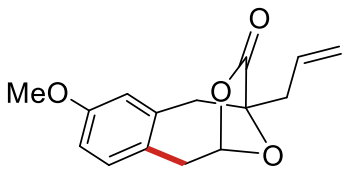

$7 \mathrm{~m}$

$\left(\mathrm{CDCl}_{3}, 400 \mathrm{MHz}\right)$

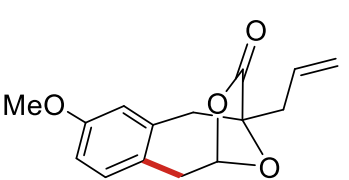

$7 \mathrm{~m}$

$\left(\mathrm{CDCl}_{3}, 100 \mathrm{MHz}\right)$

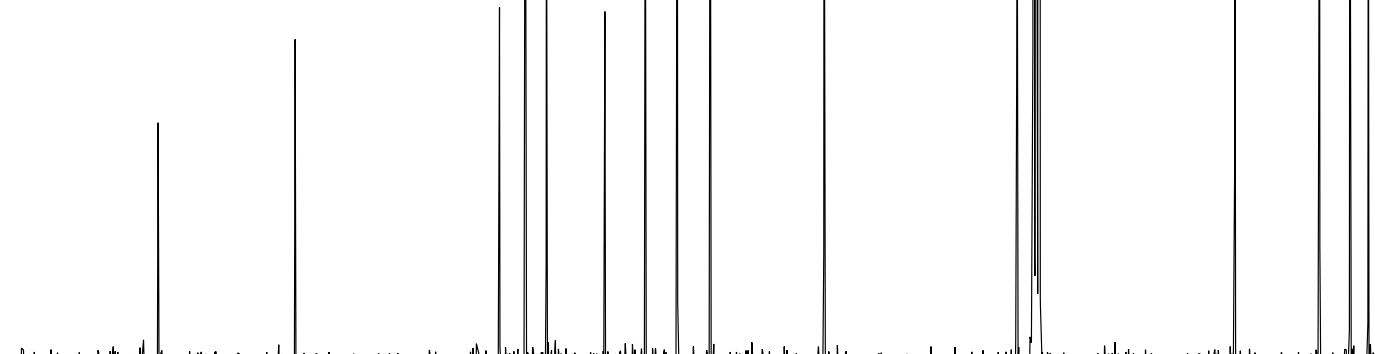




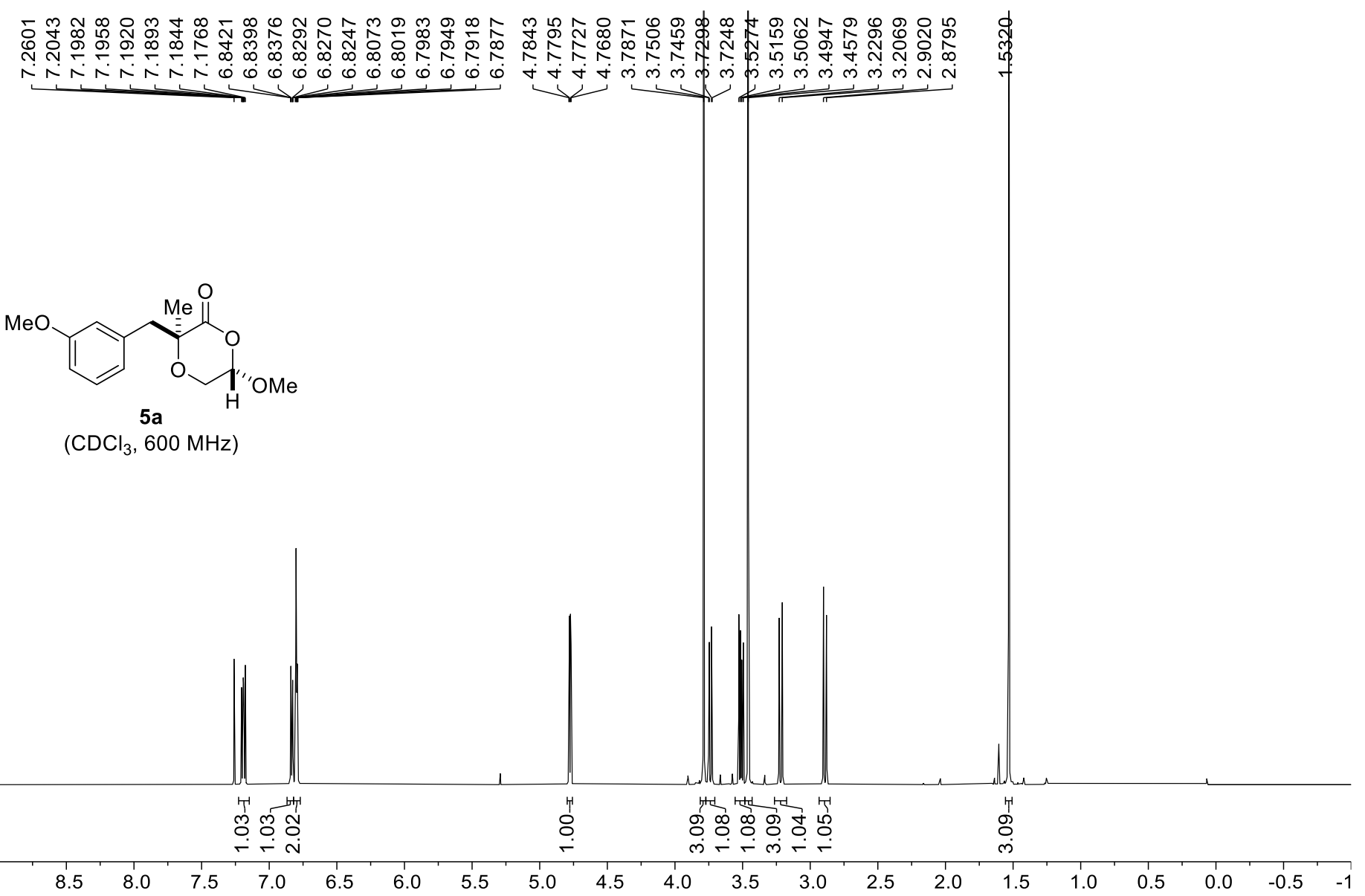

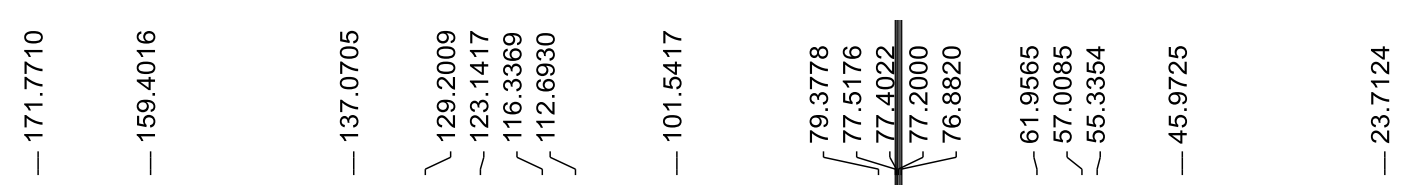

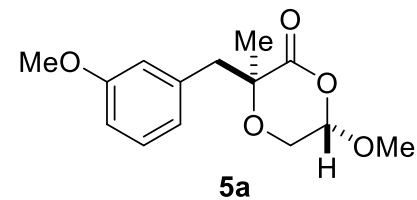

$\left(\mathrm{CDCl}_{3}, 100 \mathrm{MHz}\right)$

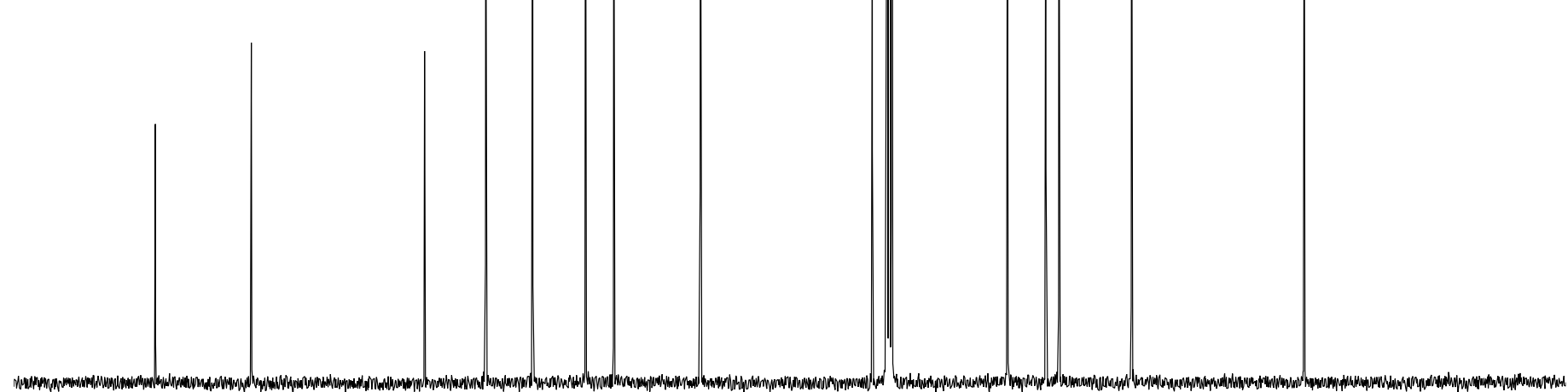

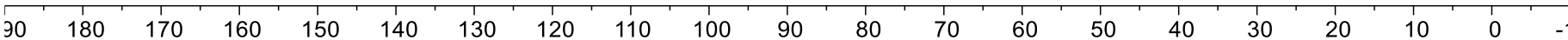




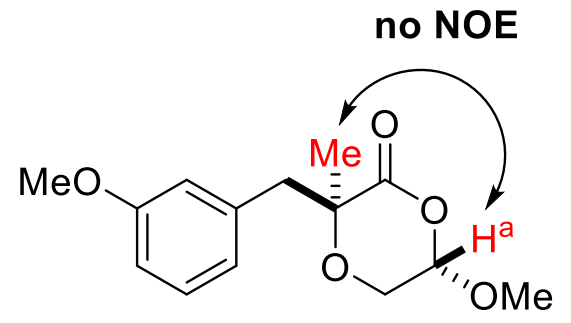

$5 a$

$\left(\mathrm{CDCl}_{3}, 600 \mathrm{MHz}\right)$

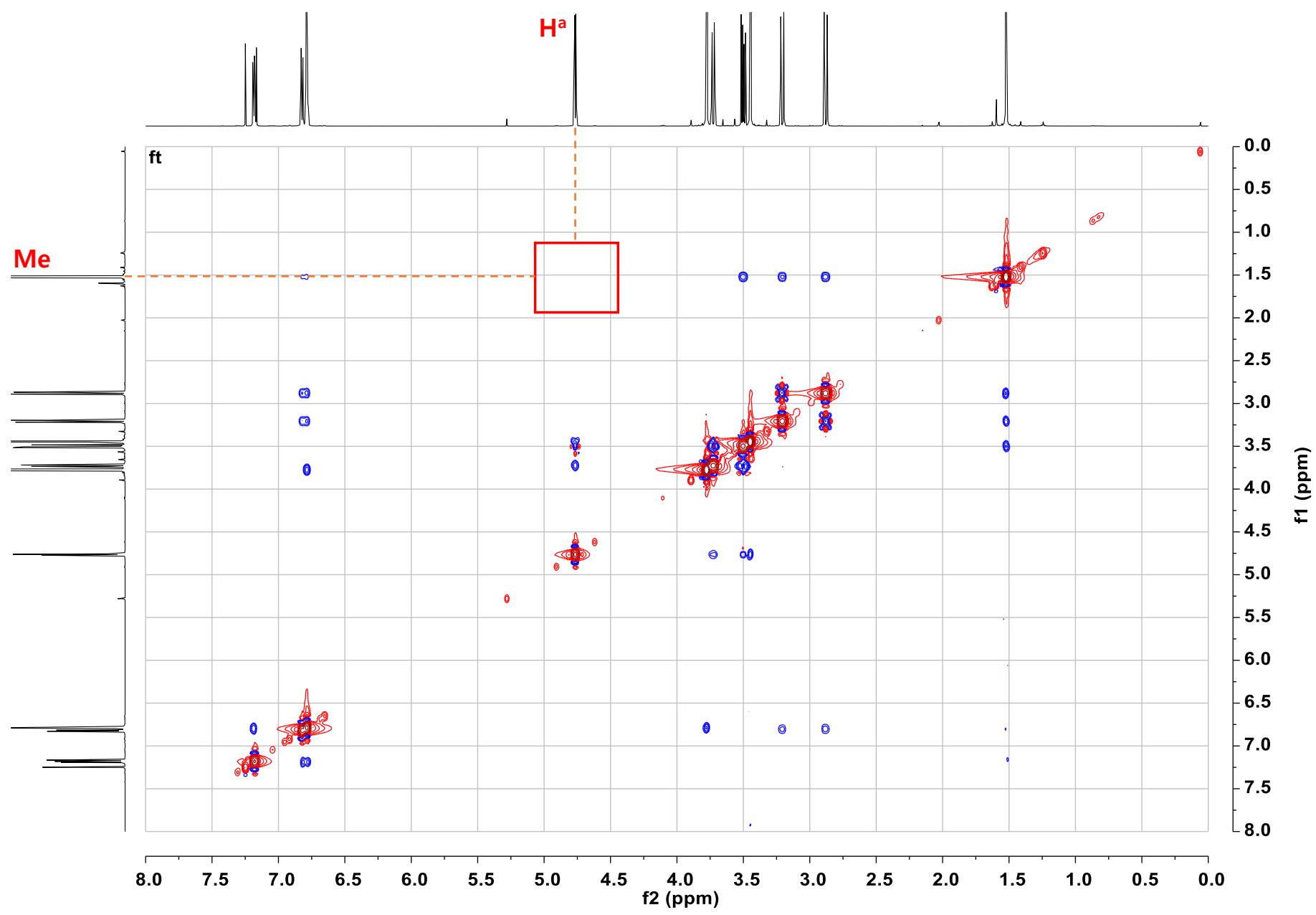




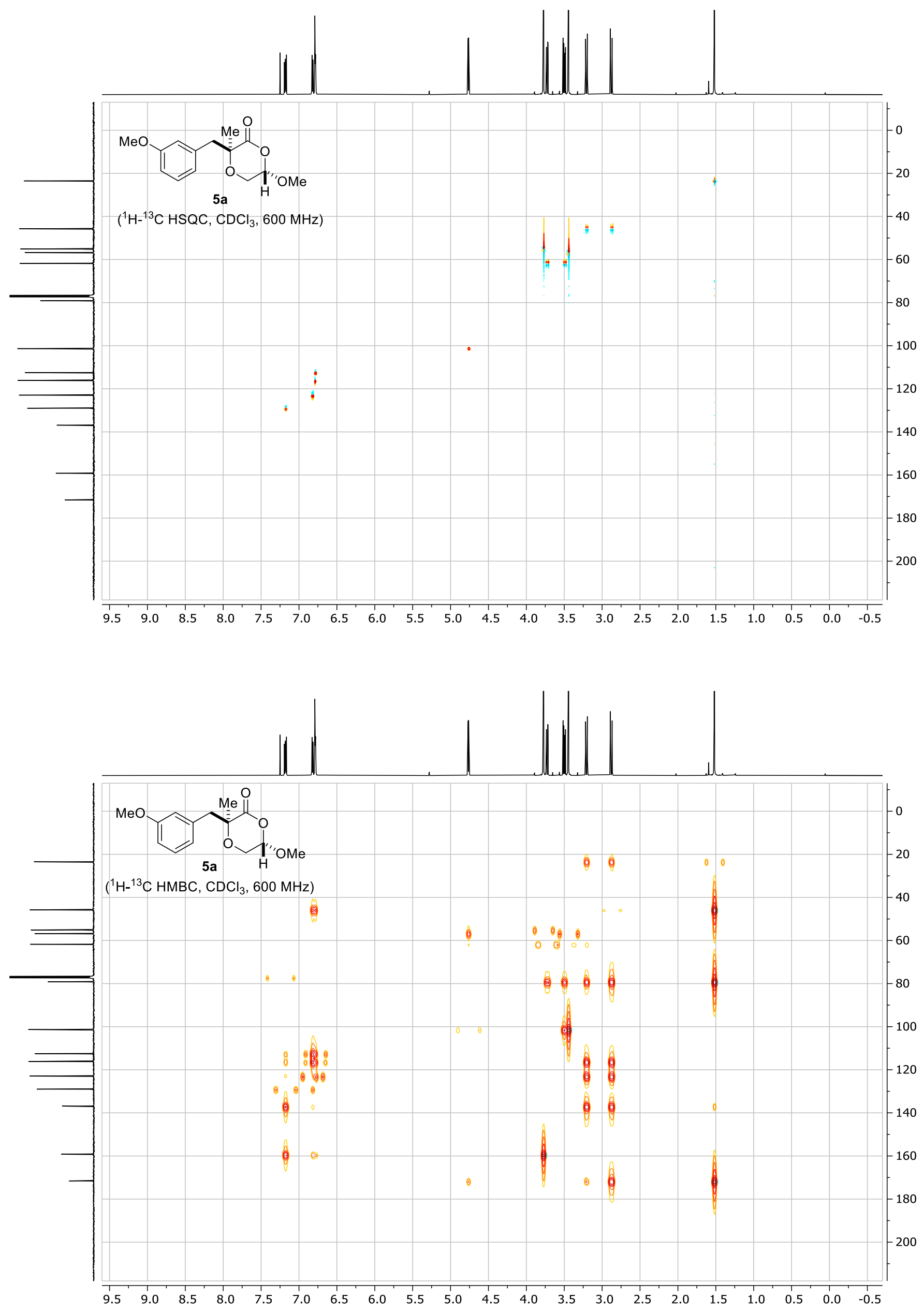


<smiles>COc1cccc(CC2(C)OC[C@H](OC)OC2=O)c1</smiles>

$\left(\mathrm{CDCl}_{3}, 400 \mathrm{MHz}\right)$

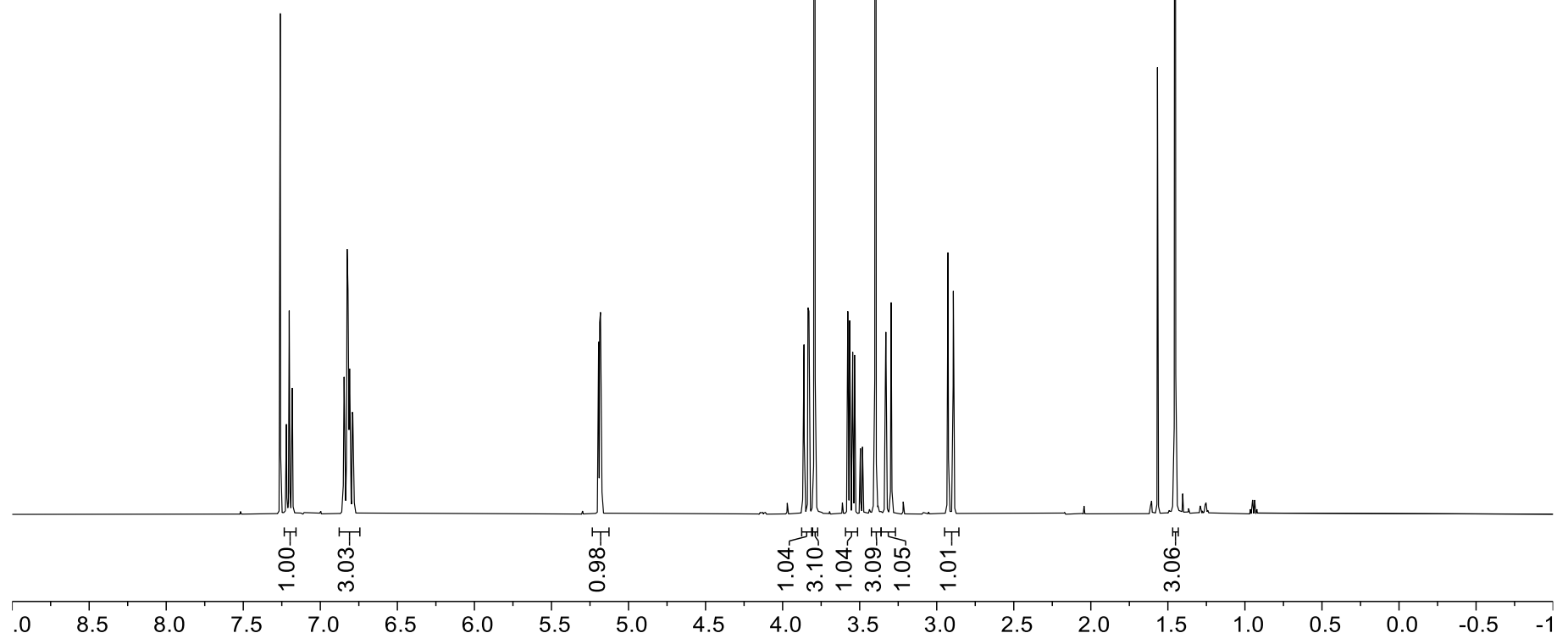

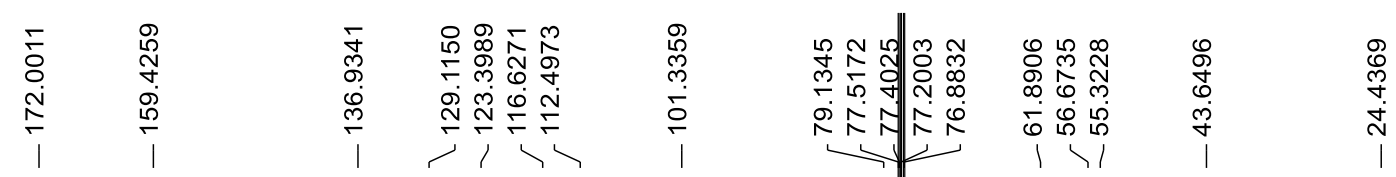<smiles>COc1cccc(CC2(C)OC[C@@H](OC)OC2=O)c1</smiles>

$\left(\mathrm{CDCl}_{3}, 100 \mathrm{MHz}\right)$

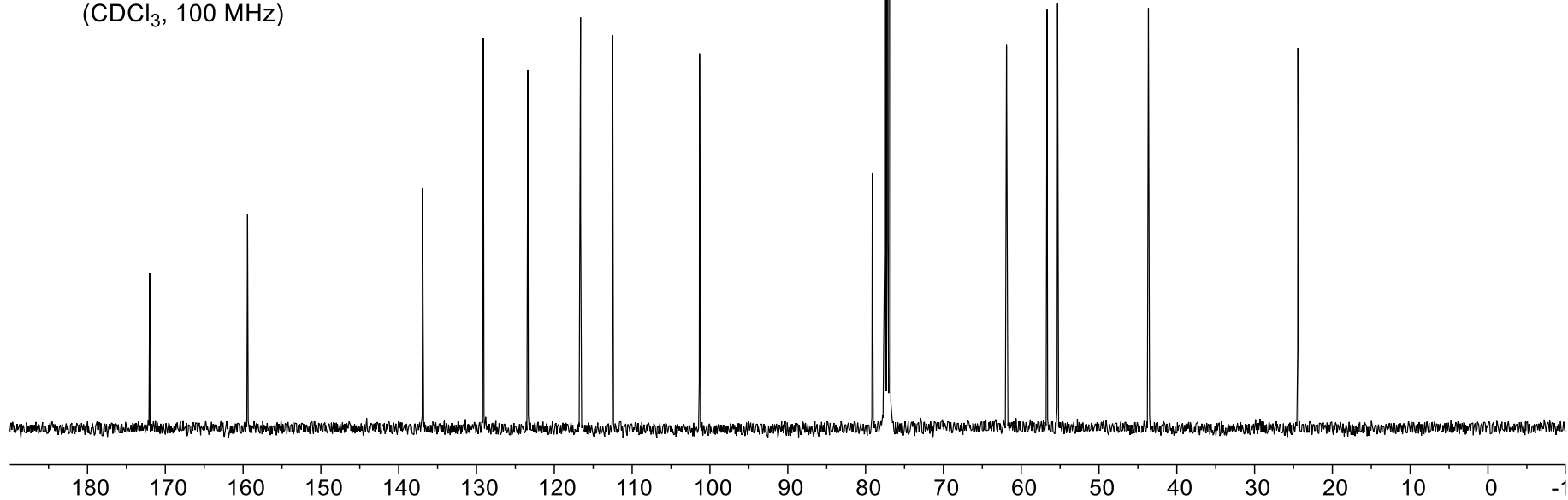




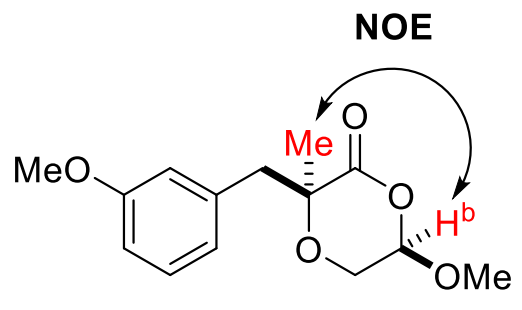

$5 b$

$\left(\mathrm{CDCl}_{3}, 600 \mathrm{MHz}\right)$

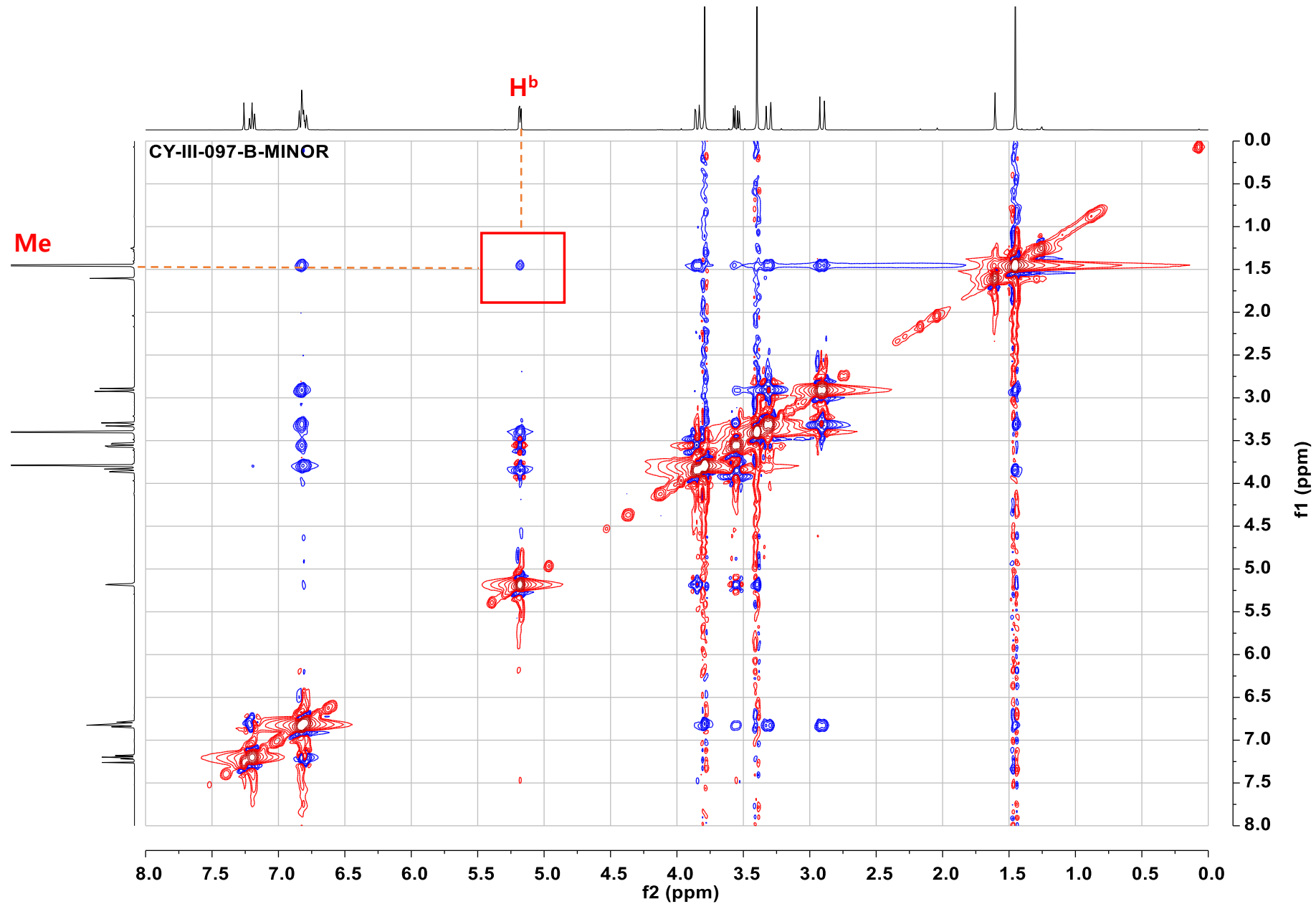

